\title{
INCREASING YIELDS AND BROADENING MARKETS: PROCESS INNOVATIONS IN THE MANUFACTURING OF ENERGY-SAVING WindOW GLAZINGS
}

\section{FINAL REPORT}

For the period 9/23/99 through 9/30/03

Principal Authors: Dr Mark Burdis and Dr Neil Sbar

Report issue date: November 2003

Revised: April 2005

DOE Award number: DE-FC26-99FT40647

\author{
Submitted by: \\ SAGE Electrochromics, Inc, \\ One Sage Way, Faribault, MN 55021 \\ Tel. (507) 331-4848 • Fax. (507) 333-0145
}




\section{Disclaimer:}

“This report was prepared as an account of work sponsored by an agency of the United States Government. Neither the United States Government nor any agency thereof, nor any of their employees, makes any warranty, express or implied, or assumes any legal liability or responsibility for the accuracy, completeness, or usefulness of any information, apparatus, product, or process disclosed, or represents that its use would not infringe privately owned rights. Reference herein to any specific commercial product, process, or service by trade name, trademark, manufacturer, or otherwise does not necessarily constitute or imply its endorsement, recommendation, or favoring by the United States Government or any agency thereof. The views and opinions of authors expressed herein do not necessarily state or reflect those of the United States Government or any agency thereof." 


\section{ABSTRACT}

The goal of this project was to develop and implement advanced thin film process technology which would significantly improve the manufacturability of both static and dynamic high performance energy saving coatings for windows. The work done has been aimed at improvements to the process that will result in increases in yield, and this was divided into four main areas, dealing with improvements in substrate preparation methods, reductions in the incidence of problems caused by particulate contamination, use of in-situ optical monitoring to improve process control, and overall system integration to enable simplified, and therefore lower cost operation. Significant progress has been made in each of the areas.

In the area of substrate preparation, the enhanced washing techniques which have been developed, in combination with a new inspection technique, have resulted in significant reductions in the number of EC devices which are rejected because of substrate problems.

Microscopic inspection of different defects in electrochromic devices showed that many were centered on particles. As a result, process improvements aimed at reducing the incidence of particles throughout the entire process have been implemented. As a result, the average number of defects occurring per unit area has been significantly reduced over the period of this project.

The in-situ monitoring techniques developed during this project have become an indispensable part of the processing for EC devices. The deposition of several key layers is controlled as a result of in-situ monitoring, and this has facilitated significant improvements in uniformity and repeatability.

Overall system integration has progressed to the stage where the goal of a closed-loop monitoring and control system in within reach, and it is anticipated that this will be achieved during the scale-up phase.

There has been a clear increase in the yield occurring over the period of this project (Sept 1999 to September 2003), which is attributable to a range of process improvements implemented as a result of this work. It is anticipated that the yield will increase further as a result of these ongoing programs. The manufacturability of these advanced glazing systems has also been significantly improved, by a variety of different measures such as in-situ monitoring, system integration, and measurements taken to reduce the incidence of defects caused by contamination. It is therefore anticipated that the transfer of this performance to the new coating equipment to be introduced during scale-up to the first manufacturing plant will be reasonably straightforward. 


\section{TABLE OF CONTENTS}

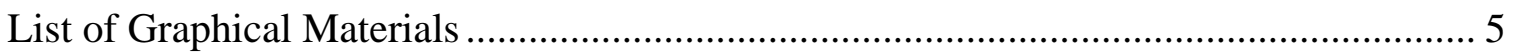

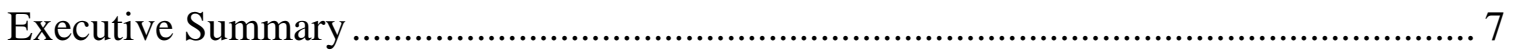

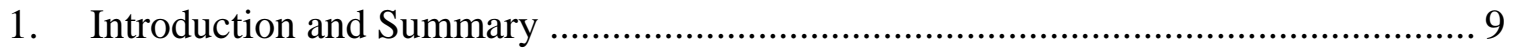

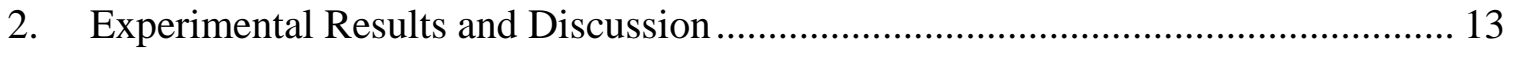

2.1 Task 1 - Substrate Inspection and Cleaning .............................................. 14

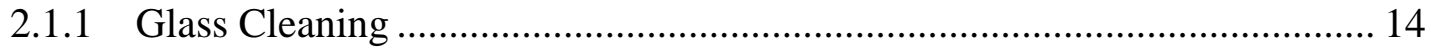

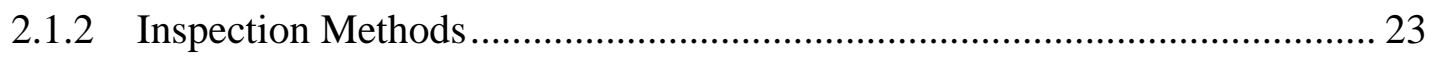

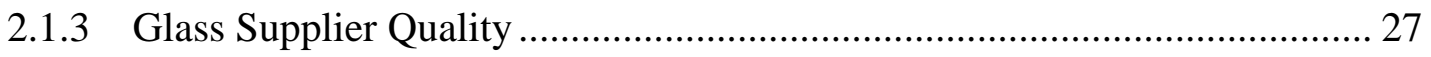

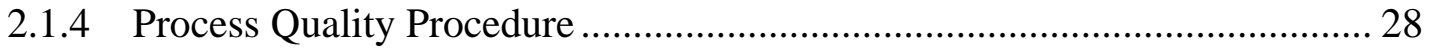

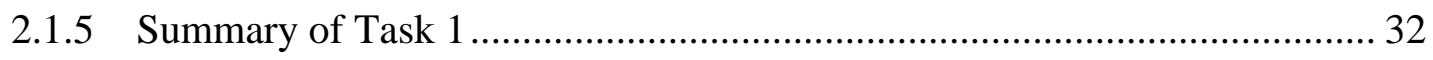

2.2 Task 2 - Identification and Minimization of Particle Generation Sources....... 33

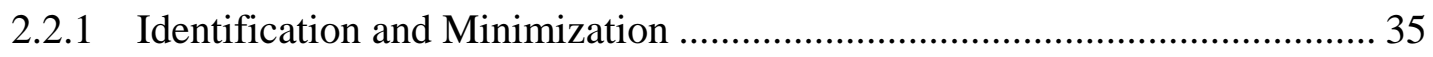

2.2.2 Manifestation of Point Defects in EC devices .......................................... 45

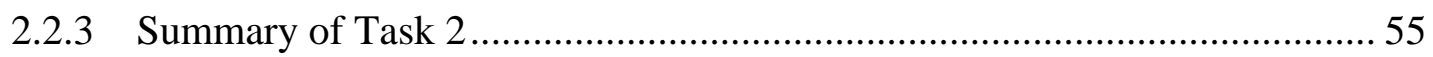

2.3 Task 3 - In-situ Optical Monitoring ........................................................ 55

2.3.1 In-situ six-point monitoring system ..................................................... 56

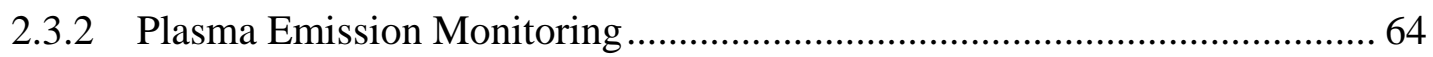

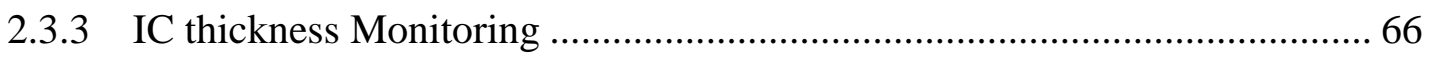

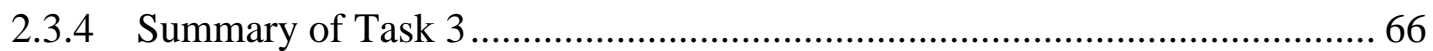

2.4 Task 4 - Integration of Inspection and Monitoring Tools ............................. 67

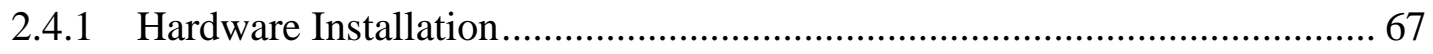

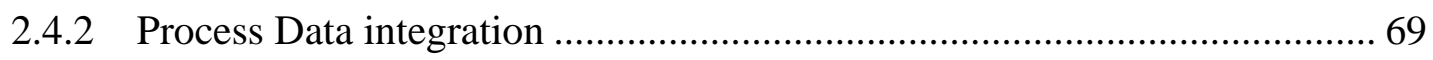

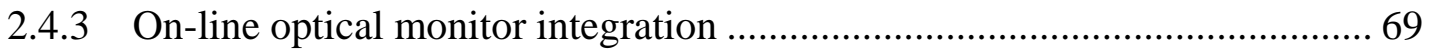

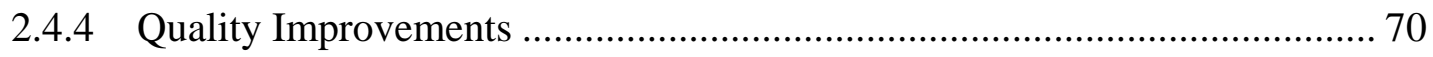

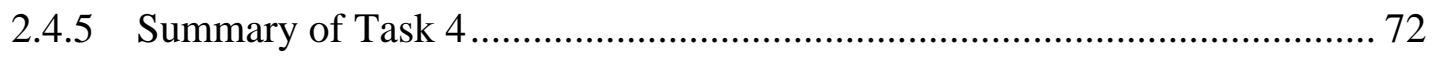

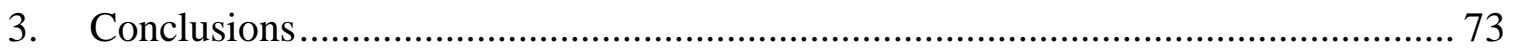

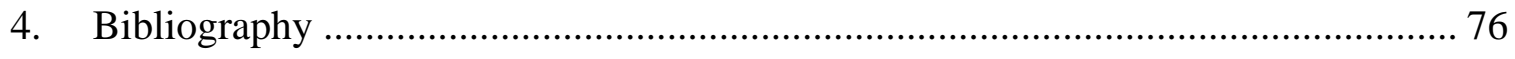

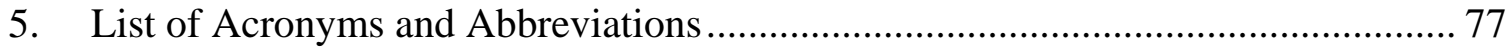




\section{LIST OF GRAPHICAL MATERIALS}

Figure 1. 1 - Schematic of the principle of operation of a SageGlass ${ }^{\circledR}$ EC window.

Figure 1. 2 - The SageGlass $^{\circledR}$ electrochromic device.

Figure 1. 3 - SAGE Pilot Line Schematic.

Figure 2.1 - Photograph of glass inspection system.

Figure 2.2 - Scanning electron micrograph of coater dust. The smallest resolved particle is less than $40 \mathrm{~nm}$ in diameter.

Figure 2.3 - Plot of the percentage run time, scheduled and un-scheduled down time for the pilot line coater. Unscheduled downtime has been reduced from around $25 \%$ at the start of the project to approximately $3 \%$ at the time of writing.

Figure 2.4 - Close-up view of a score mark in glass prior to breaking, showing significant glass damage and some loose particulate contamination. Many particles are visible near to the score, and some flakes where the glass has been removed are visible, particularly over to the left of the image.

Figure 2.5 - Examples of dark field images of sandblasted running vertically (on the left $-100 \times$ magnification) and laser isolated running horizontally (right $200 \times$ magnification) lines.

Figure 2.6 - Picture of screen printer showing the plastic shroud, and the proximity to the door discussed in the text.

Figure 2.7 - Plot of average white dot density over the last year, showing a significant reduction primarily caused by the contamination reduction measures that have been implemented.

Figure 2.8. - Tin speck on glass surface formed during the glass deposition process. The spheroid is approximately an order of magnitude larger than the electrochromic film thickness and therefore interrupts the thin film stack, creating a localized short.

Figure 2.9 - Example of an SEM photomicrograph of a glass fragment found at the center of a short.

Figure 2.10 - Picture taken through a microscope of a line short, showing a typical shape for this type of short.

Figure 2.11 - The number of hard shorts measured at initial test for each large device produced in the last year. The line is a best-fit trend line, showing the overall decrease with time. 
Figure 2.12 - The average white dot density divided into substrate preparation lots, showing the correlation of dot density with preparation lot. It is thought that the variation may be a result of uncontrolled variation seen in the tempering furnace.

Figure 2.13 - Schematic diagram of the layout of the spectrometer system. The individual channels are labeled 1-6, and the carrier is shown schematically moving under the spectrometer.

Figure 2.14 - Example of the measurement of the in-situ transmission of the CE layer during deposition.

Figure 2.15 - Dependence of the final transmission of the CE upon the initial substrate temperature.

Figure 2.16 - The transmission of CE witness pieces labeled by run number for a period of approximately five months.

Figure 2.17 - The thickness of the samples measured by the method outlined in the text, for those samples whose transmission is given in Figure 2.16.

Figure 2.18 - The value of $k(500 \mathrm{~nm})$ of the samples measured by the method outlined in the text, for those samples whose transmission is given in Figure 2.16.

Figure 2.19 - Boosted plasma emission signal from EC plasma, showing improved resolution over the unboosted signal of the transition metal lines.

Figure 2.20 - A view of folding mirrors installed in the coater to permit real time monitoring of the plasma emission profile.

Figure 2.21 - Schematic representation of the data collection set-up for the Pilot Line coater and cleanroom. Control signals are sent from the Control PC to the coater via the PLC, and the process monitor and optical monitor PCs record data returned from the coater. These data are then passed to the LAN for analysis.

Figure 2.22 - Control signal responsible for drive-speed prior to the changes discussed in the text.

Figure 2.23 - Control signal for drive-speed after improvements.

Figure 2.24 - Measurement of the thickness of one of the key layers in the EC device, both before and after the changes were made to the drive control signal.

Figure 3. 1 - Overall yield for the Pilot Line both actual and predicted. The yield figure for 2005 has been adjusted to accommodate the known equipment and logistical issues of the present Pilot Line. 


\section{Executive Summary}

The goal of this project was to develop and implement advanced thin film process technology which would significantly improve the manufacturability of both static and dynamic high performance energy saving coatings for windows. The work reported here has been aimed at improvements to the all solid state electrochromic window manufacturing process developed by SAGE Electrochromics, Inc., which will result in increases in yield and throughput. The work was divided into four main areas, dealing with initial substrate preparation, particle issues throughout the process, in-situ optical monitoring, and overall monitoring system integration. Techniques developed are generally transferable to deposition equipment dedicated to production of other glazing systems.

In the area of substrate preparation, many improvements over previously accepted 'bestpractices' have been made, including the installation and modification of a high quality washing system. An investigation of different washing techniques was carried out, yielding an optimum arrangement for reducing defects, the incidence of which has been monitored using newly developed optical inspection techniques. Another significant part of the work in the substrate preparation area was the development of an excellent working relationship with the glass supplier. This has resulted in numerous improvements to the quality of the as-received glass, and has also resulted in a process giving the best chance of maintaining the high quality glass that is required for this exacting process.

A further significant advancement in the area of substrate preparation has been the introduction of a quality program. The aim of this program is twofold: first, to ensure the highest possible level of consistency throughout all of the substrate preparation steps, and secondly, to improve the process itself by eliminating as many possible sources of contamination as possible. This is part of a larger program, designed to give the best possible chance of success in the near-future as a manufacturing operation is set-up.

Obviously, some of the measures which have been taken in the substrate preparation area impact the generation of particles, particularly in the areas of glass cutting, seaming, line isolation and bus bar application, and substantial advances have been made in the reduction of the numbers of these particles, and their propensity to find their way into the process. In addition, the issue of particle generation in the sputtering process has been addressed, and substantial progress has been made in understanding the origin of the contamination. Again, the steps that have been taken to reduce the contamination have been reflected in the improvements recorded in defect density, particularly in white dots and shorts, two of the most common defects seen in all-solid state electrochromic devices.

More success can be claimed in the area of in-situ optical monitoring. This is now a crucial and well-understood part of the process. Robust hardware has been developed, undergoing several iterations, and has been integrated neatly into the vacuum system, enabling real-time measurements as required. Furthermore, there has been an investigation into the dependence of electrochromic device properties on many of the process characteristics, and now the optical monitor is used to maintain the process at the 
optimum point. Software systems have been designed to generate information that removes the requirement for the operator to make critical processing decisions. Currently the process of integrating the output of the optical monitor into a suite of software programs is occurring, which will ultimately result in closed loop control of the whole fabrication process. A bonus development has been the discovery of a novel method for controlling the uniformity of one of the most important layers, which depends critically on real-time feedback from the optical monitoring system.

The in-situ monitoring techniques developed during this project have become an indispensable part of the processing for EC devices. The deposition of several key layers is controlled as a result of in-situ monitoring, and this has facilitated significant improvements in uniformity and repeatability.

The work on plasma emission monitoring has not progressed to the same extent, but valuable progress has been made on implementing this technique. Particular interest has been generated for linking plasma emission monitoring with the real-time uniformity control system. This could provide advance warning of any non-uniformity in the deposition process, and so allow correction of the potential problem prior to any film deposition.

The final part of the project was concerned with integration of a variety of monitoring systems with a central database. As an example, the important parameters from the sputter coater systems, such as pressure, powers, voltage, gasflow, linespeed, temperature, etc., are all recorded centrally by a dedicated process-monitoring computer. This information has been used to diagnose some existing problems, such as variations in cleanroom temperature, and inconsistencies in drive-speed. This has lead directly to several small but significant improvements. Overall system integration has progressed to the stage where the goal of a closed-loop monitoring and control system is within reach, and it is anticipated that this will be achieved during the scale-up phase.

There has been a clear increase in the yield occurring over the period of this project (Sept 1999 to September 2003), which is mostly attributable to the work done on process improvements as detailed in the report. It is anticipated that the yield will increase still further as a result of the programs that have been implemented, and it is also anticipated that transfer of this performance over to the new coating equipment will occur smoothly, as and when scale-up to the first manufacturing plant occurs. 


\section{Introduction and Summary Identification of Need and Problem Definition}

There is a need to improve the energy efficiency of building envelopes as they are the primary factor governing the heating, cooling, lighting and ventilation requirements of buildings - influencing $53 \%$ of building energy use. In particular, windows contribute significantly to the overall energy performance of building envelopes, thus there is a need to develop advanced energy efficient window and glazing systems. The annual U.S. energy use in commercial and residential buildings is approximately 34.6 Quadtrillion BTUs (Quads). Of this, 5.7 Quads is lost through inefficient window systems. ${ }^{1}$

Advanced glazing systems such as electrochromic (EC) smart windows have the capability for significant energy savings in buildings. For cooling dominated environments, the U.S. Department of Energy's DOE 2.1E simulation program estimates 20 to $30 \%$ annual energy savings for buildings with EC windows. ${ }^{2,3}$

The goal of this project was to develop and implement advanced thin film process technology that will significantly improve the manufacturability of both static and dynamic high performance energy saving windows.

In the proposal for this project, it was calculated that annual energy savings of $\sim 0.7$ Quads could be achieved nationally by implementation of electrochromic windows. Significant market penetration would be required in order to realize the estimated energy benefit. Such widespread adoption will require high performance dynamic windows at a competitive price that allows building owners to recover costs in less than five years. Consequently, the focus of this DOE project is to increase process and product yields as well as throughput for the manufacture and fabrication of energy-saving EC windows.

The following tasks were carried out to reduce device defects and improve manufacturability:

- The quality of incoming glass substrates was evaluated by identifying and classifying defects. An inspection technique was developed to screen substrates and highlight hard-to-see surface imperfections that often appear only after value added coatings have been deposited.

- Multiple sources have been identified for yield-impacting particulates that originate in the thin film vacuum coater. Processing conditions were optimized to reduce particulate generation and subsequent downtime.

\footnotetext{
${ }^{1}$ US Dept. of Energy Core Data Book, 2001, Tables 1.1.1, 1.1.2, 1.1.3, 1.2.3, 1.2.10, 1.3.3, 1.3.9.

${ }^{2}$ M.D. Rubin and R.T. Sullivan, Lawrence Berkeley National Laboratory, Personal Communication energy calculations.

${ }^{3}$ R. Sullivan, E.S. Lee, K. Papamichael, M. Rubin and S. Selkowitz, "Effect of Switching Control Strategies on the Energy Performance of Electrochromic Windows,” Proc. of SPIE International Symposium on Optical Materials Technology for Energy Efficiency and Solar Energy Conversion XIII, Freiburg, Germany, April 1994.
} 
- In-situ optical monitoring techniques were developed and implemented for real-time control of critical thin film device layers. This capability is important to achieve uniformity and increase yields.

- A data management system has been installed to collect responses from the various process measurements for analysis and feedback for overall system control.

\section{Description and Benefits of Electrochromic Windows}

Electrochromic (EC) windows represent the next generation of advanced glazing technology that will (1) reduce the energy consumed in buildings, (2) improve the overall comfort of the occupants and (3) improve the thermal performance of the building envelope. "Switchable" EC windows provide, on demand, dynamic control of visible light, solar heat gain, and glare without blocking the view. As exterior light levels change, the window's performance can be electronically adjusted to suit conditions. A schematic illustrating how SageGlass ${ }^{\circledR}$ electrochromic windows work is shown in Figure 1.1 .

SageGlass ${ }^{\circledR}$ EC glazings offer the potential to save cooling and lighting costs, with the added benefit of improving thermal and visual comfort. Control over solar heat gain will also result in the use of smaller HVAC equipment. 


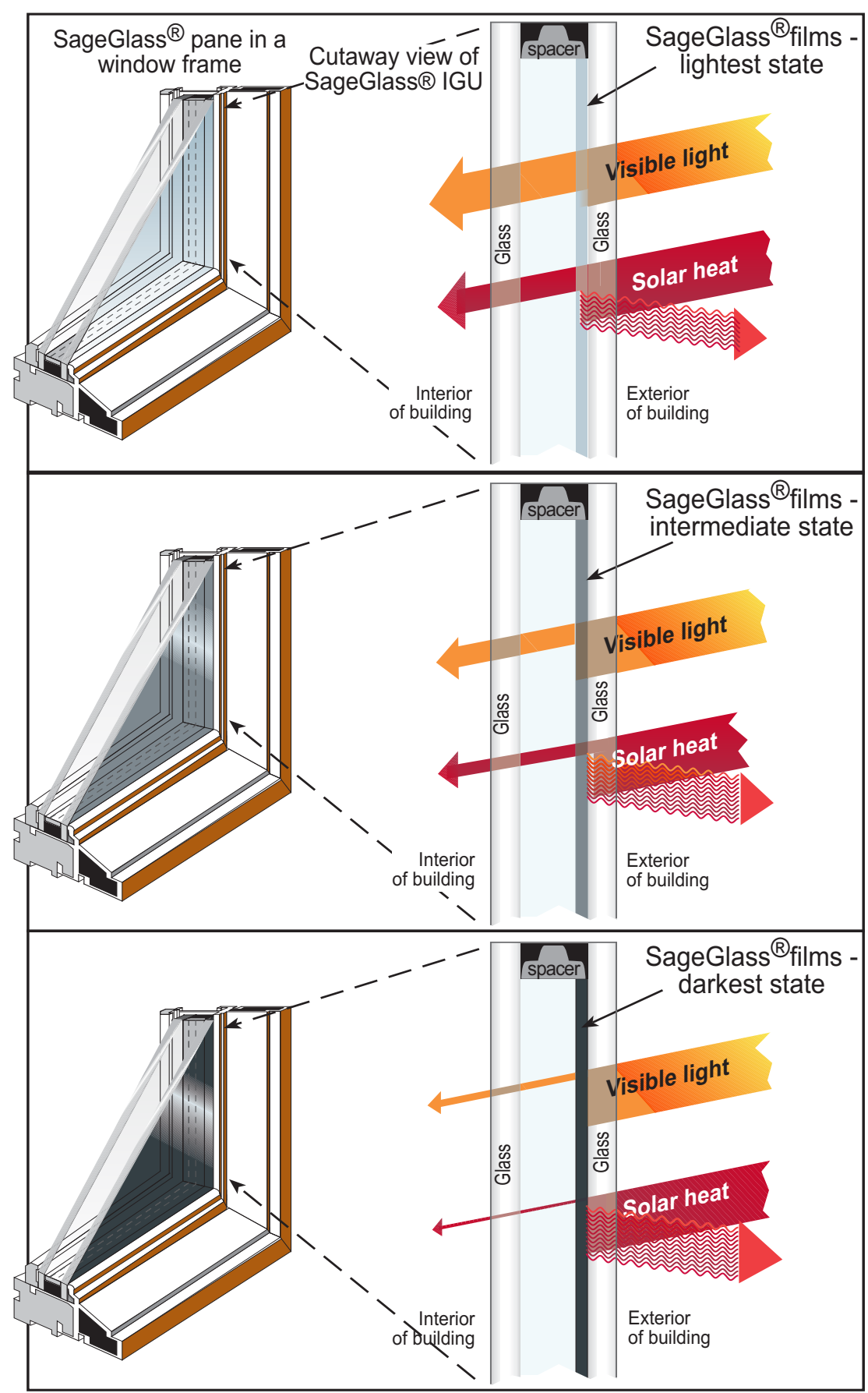

Figure 1.1 - Schematic of the principle of operation of a SageGlass ${ }^{\circledR}$ EC window.

If a step change in the energy efficiency and performance of buildings is to be achieved, there is a clear need to bring electrochromic technology to the marketplace. As noted, this project addresses the need to accelerate the widespread introduction of EC windows in buildings and thus maximize total energy savings in the US and worldwide. 


\section{EC Window Technology}

The SAGE EC device is a series of thin films that are deposited onto a glass substrate one on top of the other to form a stack ${ }^{4}$. This is shown in Figure 1.1. The outermost layers

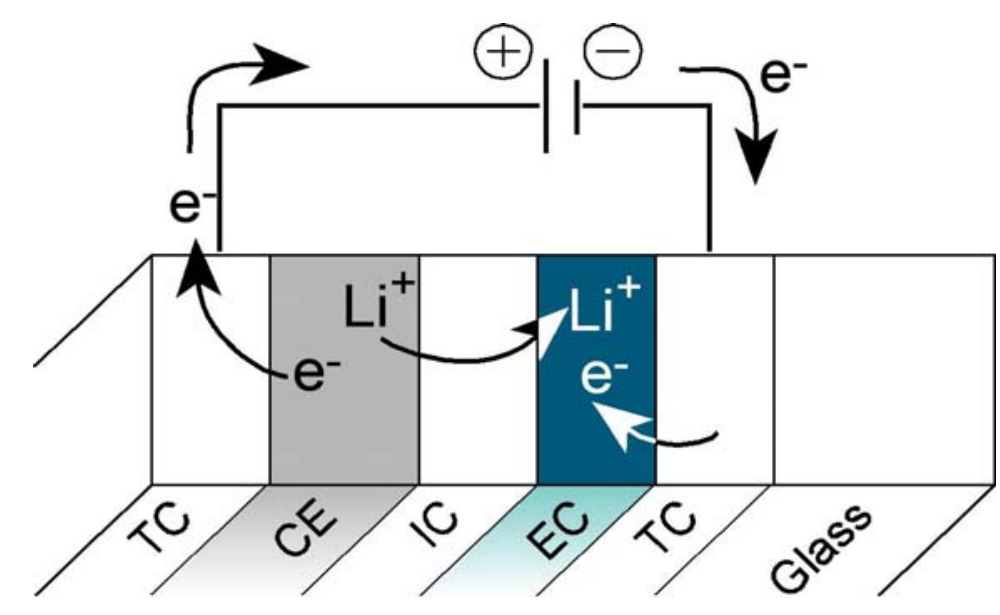

Figure 1.2 - The SageGlass ${ }^{\circledR}$ electrochromic device. are transparent conductors, which are used to apply a voltage to the active layers sandwiched between. The active layers consist of an electrochromic (EC) layer, an ion conductor (IC) layer, and a counter electrode layer (CE).

Charge, in the form of electrons

and ions, is shuttled between the CE and the EC layers, producing the bleached and colored states respectively: The electrons are passed around the outer circuit, while the ions are transported through the IC. Insertion of charge into the EC layer will cause that layer to color to a depth that depends upon the amount of charge transferred. The effect is completely reversible, and is accomplished simply by reversing the polarity of the voltage. This is shown schematically in Figure 1.2.

SageGlass ${ }^{\circledR}$ devices are fabricated on the Pilot Line which is schematically illustrated in Figure 1.3.

Incoming vendor-screened float glass with a conductive coating is received into a climate controlled storage area, and a number of operations are performed prior to thin film deposition. The glass is cut to window size, and the conductive coating is patterned to isolate areas that will later become oppositely biased electrodes. The sharp edges of the glass are removed in a process called seaming prior to the first washing operation. Next the glass is inspected for handling damage and defects. Improvements to these initial preparation steps are the subject of Task 1.

\footnotetext{
4 'Progress toward durable, cost effective electrochromic window glazings', N. Sbar, M. Badding, R. Budziak, K. Cortez, L. Laby, L. Michalski, T. Ngo, S. Schulz, and K. Urbanik, Solar Energy Materials and Solar Cells, 56 (1999) 321-341
} 


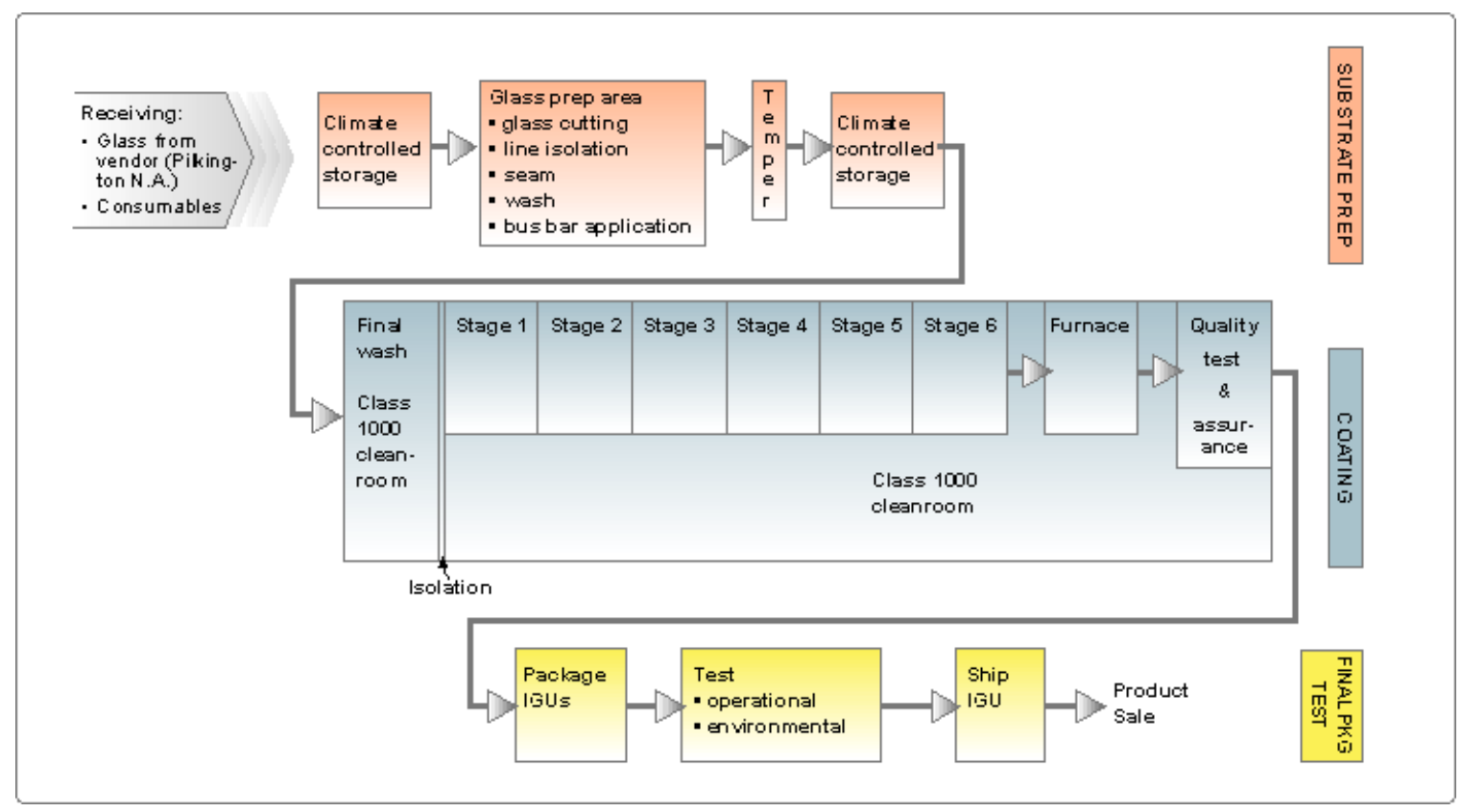

Figure 1.3 - SAGE Pilot Line Schematic.

The glass lites are tempered after bus bars are applied and subjected to a final wash before entering a class 1000 cleanroom for film deposition. Up to six physical vapor deposition (PVD) operations are carried out with appropriate heating steps. Harmful particulates need to be minimized, and this forms the subject of Task 2. In-situ control of optical properties, the development and optimization of which is the focus of Task 3 , leads to higher yields and throughput rates.

Finished EC devices are evaluated for quality and thoroughly tested for performance prior to fabrication into dual pane insulating glass units (IGUs). The IGUs are subjected to accelerated aging to screen for processing defects. Information from inspection, processing, monitoring, and testing steps has been collected and analyzed for process and product improvement, and this forms the subject of Task 4. This information forms the basis of a 'knowledge center' for the EC window production process, and is continually being modified and refined.

\section{Experimental Results and Discussion}

There now follows a discussion of the experimental results obtained during this project. This is organized by task, as described above. Each task write-up contains enough explanatory material to enable the reader to understand the results. There is a summary for each task, in which the results are discussed and placed in the context of the whole project. The material that is considered proprietary is removed to separate appendices. 


\subsection{Task 1 - Substrate Inspection and Cleaning}

Electrochromic (EC) devices require a significantly higher level of substrate quality and cleanliness than standard architectural glazing products manufactured today. The reason for this is that any defects in or on the substrate prior to EC device manufacture, such as scratches, particulate contamination, finger prints, or voids, can lead to defects which will affect the coloration behavior of the EC window, and can even lead to defects which can affect an area much larger than the defect itself. The very high contrast ratio between the colored and clear (or bleached) states means that defects that would not be visible on a standard glazing product are highly visible when the EC device is colored, and hence the requirement for substrate cleanliness is much more rigorous for EC device manufacture than for other glazing manufacture.

The substrate used for SageGlass ${ }^{\circledR}$ EC devices is glass coated with one type of transparent conductor (TC). The coating is only available from a limited number of manufacturing sources. It is clearly important to understand the manufacturing process to try to control the potential causes of defects in the coating.

The major issue in this task is to understand the origin of substrate imperfections that can have a deleterious effect on the finished coated glass product. These imperfections may be intrinsic to the substrate, or may be introduced during subsequent processing. The objective of the task, therefore, is to find methods of identifying glass substrates with defects that can cause EC device deposition problems, and the progress made on this task is discussed in detail here. We will concentrate on the areas of glass cleaning, inspection methods, supplier interactions, and the process quality procedures that have been put in place.

\subsubsection{Glass Cleaning}

As indicated, it is important that substrates used for EC device manufacture are as clean as possible. The glass suppliers are located in different parts of the country, and so the glass must be packaged and shipped after it is manufactured. There are well known problems associated with the storage and shipping of glass ${ }^{5}$. These include corrosion of the surface (also known as staining) as a result of the glass getting wet, as well as the possibility of two adjacent glass lites scratching each other and even getting stuck together. This has resulted in the development of a packaging technology which (a) keeps the surfaces of the glass separated using small polymeric beads, and (b) prevents chemical corrosion of the glass surface by the application of an organic acid.

It is also not economically feasible or practical to keep the glass completely clean during shipping, as would be the case when transporting semiconductor manufacturing materials. Indeed, as noted previously, the glass is already coated with materials

\footnotetext{
${ }^{5}$ M.Feldman, R. Weissman, 'Initial stages of float glass corrosion', Journal of Non-Crystalline Solids, 218 (1997) 205-209
} 
designed to prevent scratching and weathering. It is therefore essential that there is an effective in-house cleaning process.

The development of the flat glass cleaning process, utilizing a modified industry standard flat glass washer, is discussed first. Then the results of work carried out using chemical etching in order to obtain better substrate cleanliness are discussed.

\subsubsection{Flat Glass Washer}

Prior to the beginning of this project, the standard approach used by SAGE to glass cleaning was a complex and time-consuming process, involving hand-scrubbing and rinsing in several different tanks of deionized (DI) water. This was acceptable (if labor intensive) for small sizes of glass, up to about 10 " $\times 12$ ”, but would be completely impractical for the larger sizes needed to be processed during the course of the scale-up to practical window sizes.

The investigation was started using a domestic dishwasher for glass cleaning, and the performance was found to be perfectly acceptable. This led to a search for manufacturers of commercial-scale 'dish-washer'-style washing systems. Several were located, but it was found that the cost for custom design and manufacture of such a system was prohibitive at this stage of the overall process development.

As a result, effort was focused on determining whether an industry standard flat glass washer would be acceptable for the purpose. This began by locating a number of flat glass washers in the area, and several samples of glass were washed in each of them. Simple inspection of the washed glass using optical microscopy revealed various degrees of cleanliness, mostly dependent on the level of cleanliness of the washer itself and its surrounding environment. It was therefore clear that a machine was required with cleaning qualities superior to those generally available in the glass industry, and that particular attention had to be paid to its maintenance. The necessity for using highly conditioned water during all the steps of the washing process, not only during the final rinse, was also demonstrated.

To address these needs, a used flat glass washer was acquired for exclusive use with the pilot line, and a sophisticated DI water system incorporating particle filtration down to 0.2 microns was installed.

Upon delivery, the flat glass washer consisted of two wash sections, a rinse section and a low velocity hot air drying section. The wash sections used several spray bars directly above the glass to introduce the water into the system. Cleaning was achieved by a combination of brushes and sponge rollers on both surfaces of the glass, and the glass was driven through the washer using top and bottom pinch rollers.

The machine had been in storage for several years and was in need of repair. Many parts were either missing or unusable. Rust was present in several wetted areas as well as on the frame. A thorough cleaning was necessary to remove dirt, rust and corrosion. The 
wiring in the control console was found to be substandard, necessitating much work to bring it up to code.

It was decided that the cleaning process was crucial to the success of the project. Much of the semiconductor and flat panel display industry spend a high proportion of their time and effort on cleaning of surfaces before coating takes place. As a result of benchmarking the process against those, it was decided that excellent water quality would be required.

Minnesota water is extremely hard; with large quantities of calcium and magnesium salts and enough dissolved iron to interfere with the operation of most water purification equipment. The DI water system was designed with a four-step treatment plan. The four treatment steps are: pretreatment with softening and filtration, reverse osmosis (RO) which removes $95 \%$ of the dissolved solids, deionization and filtering which removes suspended particles.

The pretreatment step uses a commercial water softener to replace metallic ions (i.e. Ca, $\mathrm{Mg}, \mathrm{Fe}$ ) in the water with sodium ions. The softener uses a conventional ion exchange resin that is flushed with salt water to regenerate it. The water next flows through an activated carbon filter to reduce levels of suspended matter and remove organics that might clog the RO membrane.

The RO component separates the pretreated water into two streams. $90 \%$ of the water is forced through a porous membrane by high pressure. Dissolved ions in the water are too large to go through the membrane and are flushed down the drain along with the remaining $10 \%$ of the water.

Water to the DI system is supplied from the RO system. The first part of this is a tank with two types of ion exchange resins in it. The cation resin exchanges positive ions such as $\mathrm{Na}^{+}$and $\mathrm{Ca}^{+}$with $\mathrm{H}^{+}$ions. The anion resin exchanges negative ions such as $\mathrm{Cl}^{-}$and $\mathrm{SO}_{4}{ }^{-}$with $\mathrm{OH}^{-}$. The $\mathrm{H}^{+}$and $\mathrm{OH}^{-}$combine to form water. After the ion exchange process, the water is quite free of contaminants but some residual organic material may remain. A high intensity short-wave ultraviolet irradiation system is used to break up any organic material left in the water and to kill any micro-organisms that may be in the water supply.

The deionized water is circulated through a storage tank and a loop of piping that comes near the point-of-use sites in the production area. In this loop are a set of coarse and fine filters that remove any trace amounts of particulate matter that may remain.

The resistivity of water is a convenient and accurate method of measuring the amount of dissolved material in solution. The water we produce has a resistivity between 16 and $17.5 \mathrm{M} \Omega \mathrm{cm}$. $16 \mathrm{M} \Omega \mathrm{cm}$ water has about $0.002 \mathrm{ppm}$ dissolved solids. $18.3 \mathrm{M} \Omega \mathrm{cm}$ is the theoretical maximum for pure water. The supply water to the Pilot Line has about $400 \mathrm{ppm}$ of dissolved hardness. Anything greater than $180 \mathrm{ppm}$ dissolved hardness is considered "very hard". 
Upon start-up the particle contamination levels in the DI water were measured and the data shown in Table 2.1 obtained:

\begin{tabular}{|l|l|l|l|l|l|}
\hline & 0.5 & 1 & 5 & $>10$ & Particle size (micron) \\
\hline Supply water & $8.3 \times 10^{6}$ & $2 \times 10^{6}$ & 17,400 & 2,800 & \multirow{2}{*}{ Particles per liter } \\
\hline Loop DI water & 2,000 & 900 & 20 & 0 & \\
\hline
\end{tabular}

Table 2.1 - Particle size analysis for water before and after filtration

This meets the standards of ASTM Electronics grade water type E-11.

The initial modifications to the washer included removing and replacing all materials from the wetted area that would corrode in the presence of water. Experience had shown that the products of such corrosion could contaminate the water and eventually end up on the surface of the glass being cleaned. Next, all the stainless steel parts in the wetted part of the washer were removed and Teflon coated. Brushes and rollers were also replaced with new ones, which were thought less likely to leave deposits on the glass surface. An external pre-wash section at the front of the washer was added. The purpose of this was to remove the plastic interleaving material present on the glass as it is received from the manufacturer. It is important to remove this prior to the glass entering the washer, because hot water in the cleaning section can cause this material to soften and clump together contaminating the interior of the machine. It can then re-deposit onto subsequent glass lites, leading to defects in finished devices. It was necessary to do this operation immediately before entering the washer itself, as washing the interleaving material off and allowing the glass to dry could lead to staining problems.

The next series of modifications to the washer included three main areas: (1) Adjustment of the brush height to determine the optimum contact force - too little force will result in poor cleaning, but too much will cause the brush material to be abraded by the rough tin oxide surface, potentially leaving deposits on the glass surface; (2) Addition of sponge rollers behind the brushes in the two wash sections, which was designed to aid in removal of small particles on the surface of the glass; and (3) Experimentation with detergents in the cleaning process.

Previous experience with outside vendors has demonstrated that aggressive scrubbing by the brushes can leave deposits on the substrate surface, which although invisible can become visible on the electrochromic device. An experiment was constructed to determine the optimum amount needed for cleaning. Using a simple adjustment mechanism, brush pressure against the substrate was increased incrementally until marks began to appear on devices. Several substrates were cleaned at each setting to provide adequate statistical data. The cleanliness of the glass was judged by counting the number of particles present after washing. This was compared to the baseline, established with the hand washing process. 
Experiments with sponge rollers did not conclusively demonstrate improved performance although the initial microscopic inspections of the substrate surface indicated fewer particles. The sponge rollers clean the glass by wiping the surface much like brushes. Increased contact area generated through a continuous, soft sponge helps to loosen particles and other contaminants adhering closely to the substrate surface. However, these rollers may also exacerbate a problem that was seen early on in the development process, where finished devices showed marks that were characteristic of 'skid' marks from the top drive rollers. The original set-up of the machine used a pinch-roller system with rollers top and bottom to drive the glass through the washer. The problem was that the top roller could slip against the glass if there was a significant frictional force opposing the glass motion, leaving marks that were visible in the finished EC device. The wiping action of the sponge roller placed more drag on the substrate as it moved through the washer. This had to be overcome by applying more friction with the top drive rollers, which lead to exaggerated marking. As a result, it was decided to remove the sponge rollers from the wash section completely. The sponge roller in the drying section remained because this roller exerted much less pressure on the glass being washed, and did not cause it to get caught in the washer or cause the rollers to slip.

The efforts to eliminate marking of the substrate surface by the drive rollers in the glass washer were largely unsuccessful. It was therefore decided to completely remove the top rollers from the washer.

Obviously, this reduced the driving force on the substrate and necessitated raising the sponge rollers (in the drying section) so that they no longer contacted the glass. (Without the top rollers, there is not enough frictional force to move the glass through the sponge rollers and so the glass would have become caught in the washer). The brush contact pressure was also reduced to compensate for the loss of top roller friction. It was seen that the cleaning efficiency was not significantly reduced, and the marks that were attributed to the rollers were subsequently absent from finished EC devices.

A further experiment with modified top rollers was carried out. In this trial, the rollers were modified so that only a small area of the roller was in contact with the glass, corresponding to a region at the edges of the glass where there is no active device area, and so there was no issue with potential marking. This re-introduction of the top drive rollers allowed experimentation with increases in pressure exerted by the brushes. There were two issues: first, the brushes did not seem to make a significant difference to the overall level of cleanliness achieved, and second, the modification to the rollers was not $100 \%$ reliable. For these reasons, the top rollers and brushes were removed from the washer, and this is how the system is currently operated.

Experiments done many years ago while developing the hand washing process for small substrates have demonstrated the effectiveness of detergent as a cleaning aid. The detergent used in the old process produces significant amounts of foam, and was therefore not compatible with the new spray equipment in the flat glass washer. An effort was therefore made to find a suitable replacement. Samples were obtained after discussions with vendors and experiments were carried out on small substrates. 
Several test pieces were cut from each big lite of substrate glass then divided into groups. Samples from each large lite were included in each group. This ensured an equal challenge for each detergent sample. Next, all samples were processed according to standard substrate preparation procedures. A home-style dishwasher was used to do the cleaning. Results were initially judged via particle counts on the cleaned substrates while final judgment was based on the electrochromic device appearance. In addition to helping us find a replacement detergent, the experiments re-confirmed the need for scrubbing of the substrate surface. None of the detergents tested were able to adequately dislodge and remove particles without some form of mechanical action.

In the end, detergents were not added to the flat glass washer, because we found that hot, filtered, DI water gave adequate results and also the hardware for introducing detergents was not fully installed. There was also concern that the detergent could potentially get into various parts of the washer, and be very difficult to remove by rinsing and thus give some potential contamination issues. It is still believed, however, that addition of an appropriate detergent will be beneficial, and we are trying to determine the best way to introduce it without causing other problems.

The next phase of washer modifications concerned the drying section of the washer. Originally, the flat glass washer used a sponge roller in the drying section to remove the bulk of the rinse water followed by a low-velocity hot air evaporative process to dry the substrate glass after washing. However, if the rinse water was not $100 \%$ clean and the debris not completely removed, contamination can obviously precipitate out during drying and remain on the surface of the glass. The inspection technique used to filter out defective substrates revealed a contaminant that would sometimes be seen on finished devices and by virtue of its physical shape was traced to the sponge roller used for drying the substrates. This confirmed the suspicion that this sponge roller could reintroduce contaminants onto a previously cleaned substrate.

Using air knives to push the water off is known to be a much better method for drying, and would therefore improve the quality of the cleaning and could even allow introduction of detergents without leaving soap residue on the substrates. The sponge roller in the drying section was removed, and the drying section modified to produce a high velocity air stream that pushed the rinse water off the substrate instead of evaporating it. The first attempt at modification to the drying system probably pushed the bulk of the water off, but left a small amount behind, which could then evaporate rapidly. This small amount still had the potential to leave contamination upon drying. A more efficient, higher velocity blow-off system that had the ability to completely shear the water off the surface and with it any dissolved or suspended contamination was therefore identified. This was installed and is currently the standard method of drying the substrates.

To summarize, a used industry standard flat glass washer has been acquired and modified to significantly improve its performance. This has included some materials modifications, particularly within the wetted section of the washer, as well as significant modifications to the drying section. A pre-wash section has been added, and several experiments performed to determine the optimum arrangement of drive rollers and 
brushes for this particular application. The work in this area has ensured that specifications for the washer that will be purchased for the next stage of development can be determined.

\subsubsection{Chemical Etching}

One of the difficult to resolve substrate cleanliness issues has been track marks. These are linear marks made by the glass supplier during manufacture. They are generally invisible prior to device processing, but cause areas that color differently to the rest of the EC device. These areas are usually straight lines approximately 4-6 $\mathrm{mm}$ in width running the full length or width of the device. By careful monitoring of the position of each glass substrate during manufacture, and correlation of the positions of the track marks seen, the origin could be determined. Unfortunately, although their occurrence could be minimized, they could not be completely removed as the elements that lead to track marks are an intrinsic part of the manufacturing process.

Normal washing has little effect on these marks (along with other marks such as fingerprints, wipe marks, and glove marks to a lesser extent), so the effectiveness of chemical etching was investigated for mark removal prior to device processing. The following sections document the work in this area, initially using cold acid or alkali etching, and later using hot acid and alkaline etchants.

\subsection{Cold acid and cold caustic solutions}

An experiment was designed in which specially 'marked' substrates were treated with a variety of acid or alkali solutions for different times. For each sample, half of the area was immersed in the solution, while the other half remained unetched to act as a control. The samples were then fabricated into EC devices, and the switching behavior examined to determine the efficacy of removal of the various different types of marks.

Preparation of the specially marked substrates proceeded as follows:

1. The substrates were washed ${ }^{6}$, inspected and all the original defects mapped.

2. A template was made to locate all purposely made "marks" so that all markings could be reproduced at exactly the same position on each substrate.

3. Substrates were marked according to the template and included the following defects:

- Latex glove mark

- Fingerprint

\footnotetext{
${ }^{6}$ These samples were 10 " $\times 12$ " in size, and were washed in a domestic dishwasher to ensure that each washing process was carried out as repeatably as possible.
} 
- Flat glass washer roller mark

- Dried water drop

- Knife scratch

- Debris from glass carrier used in the pilot line sputter coater

- 3" diameter suction cup mark

- Metal mark

- Plastic mark

- Adhesive side of a cork spacer

4. Substrates were inspected again and the visible markings documented

5. Substrates were then chemically etched, inspected again, and re-washed.

6. Substrates were then processed into EC devices, and then inspected for evidence of the removal or otherwise of the various markings described above.

The types of solution and time of the chemical etching is listed below:

- $\quad 0.1 \mathrm{M}$ Potassium hydroxide $(\mathrm{KOH})$ for 12 hours

- $\quad 0.1 \mathrm{M}$ Potassium hydroxide $(\mathrm{KOH})$ for 24 hours

- $\quad$ 0.1M Nitric Acid $\left(\mathrm{HNO}_{3}\right)$ for 12 hours

- $\quad$ 0.1M Nitric Acid $\left(\mathrm{HNO}_{3}\right)$ for 24 hours

- $0.5 \mathrm{M}$ Potassium hydroxide $(\mathrm{KOH})$ for 12 hours

- 1.0M Nitric Acid $\left(\mathrm{HNO}_{3}\right)$ for 12 hours.

Each substrate was washed before and after dipping took place to minimize contamination, and the substrates were inspected before and after dipping to monitor the effect of any treatment the glass received. This was possible because only half of each sample was immersed in the etchant, so the treated and untreated areas could be easily distinguished.

Upon inspection after etching, it was obvious that the treated substrates showed an etch line at the middle point of the glass. Inspection of the substrates that had been exposed to high concentration etching using $\mathrm{KOH}$ for 12 hours (sample set 5) indicated a complete removal of the washer roller mark and $50 \%$ to $75 \%$ removal of all other non-scratch defects. Low concentration etching using $\mathrm{KOH}$ for 24 hours (sample set 2) showed little effect on marks; only the fingerprint and the suction cup mark appeared to have marginally reduced in intensity. Etching with low concentration $\mathrm{KOH}$ for 12 hours did not affect the defects. Treatments using the nitric acid did not affect any of the defects as 
seen during inspection. In addition, no difference outside the normal range was seen in the sheet resistance of the TC after etching for either the acid or the alkaline etches.

\begin{tabular}{|l|c|c|c|c|c|c|c|c|}
\hline \multirow{2}{*}{$\begin{array}{l}\text { Substrate } \\
\text { marks }\end{array}$} & \multicolumn{7}{|c|}{ Defect present in finished EC device? } \\
\cline { 2 - 9 } & $\begin{array}{l}1.0 \mathrm{M} \\
12 \mathrm{hrs}\end{array}$ & $\begin{array}{c}0.1 \mathrm{M} \\
12 \mathrm{hrs}\end{array}$ & $\begin{array}{c}0.1 \mathrm{M} \\
24 \mathrm{hrs}\end{array}$ & $\begin{array}{c}0.1 \mathrm{M} \\
12 \mathrm{hrs}\end{array}$ & $\begin{array}{c}0.1 \mathrm{M} \\
24 \mathrm{hrs}\end{array}$ & $\begin{array}{l}0.5 \mathrm{M} \\
12 \mathrm{hrs}\end{array}$ & \multicolumn{2}{l|}{$\begin{array}{l}\text { Marked \& washed in } \\
\text { alkaline detergent in } \\
\text { dishwasher }\end{array}$} \\
\hline Glove mark & $\mathrm{X}$ & $\mathrm{N}$ & $\mathrm{N}$ & $\mathrm{X}$ & $\mathrm{N}$ & $\mathrm{N}$ & $\mathrm{N}$ & $\mathrm{N}$ \\
\hline Fingerprint & $\mathrm{X}$ & $\mathrm{N}$ & $\mathrm{N}$ & $\mathrm{X}$ & $\mathrm{N}$ & $\mathrm{Y}$ & $\mathrm{N}$ & $\mathrm{N}$ \\
\hline $\begin{array}{l}\text { Dried water } \\
\text { drop }\end{array}$ & $\mathrm{X}$ & $\mathrm{N}$ & $\mathrm{N}$ & $\mathrm{X}$ & $\mathrm{N}$ & $\mathrm{N}$ & $\mathrm{N}$ & $\mathrm{N}$ \\
\hline Knife scratch & $\mathrm{X}$ & $\mathrm{Y}$ & $\mathrm{Y}$ & $\mathrm{X}$ & $\mathrm{Y}$ & $\mathrm{Y}$ & $\mathrm{Y}$ & $\mathrm{Y}$ \\
\hline Coater debris & $\mathrm{X}$ & $\mathrm{N}$ & $\mathrm{Y}$ & $\mathrm{X}$ & $\mathrm{Y}$ & $\mathrm{N}$ & $\mathrm{N}$ & $\mathrm{N}$ \\
\hline Suction cup & $\mathrm{X}$ & $\mathrm{N}$ & $\mathrm{Y}$ & $\mathrm{X}$ & $\mathrm{Y}$ & $\mathrm{N}$ & $\mathrm{N}$ & $\mathrm{N}$ \\
\hline Metal mark & $\mathrm{X}$ & $\mathrm{N}$ & $\mathrm{Y}$ & $\mathrm{X}$ & $\mathrm{Y}$ & $\mathrm{Y}$ & $\mathrm{Y}$ & $\mathrm{N}$ \\
\hline Plastic mark & $\mathrm{X}$ & $\mathrm{N}$ & $\mathrm{N}$ & $\mathrm{X}$ & $\mathrm{N}$ & $\mathrm{N}$ & $\mathrm{N}$ & $\mathrm{N}$ \\
\hline Cork spacer & $\mathrm{X}$ & $\mathrm{N}$ & $\mathrm{N}$ & $\mathrm{X}$ & $\mathrm{N}$ & $\mathrm{N}$ & $\mathrm{N}$ & $\mathrm{N}$ \\
\hline Track mark & $\mathrm{X}$ & $\mathrm{Y}$ & $\mathrm{Y}$ & $\mathrm{X}$ & $\mathrm{Y}$ & $\mathrm{Y}$ & $\mathrm{Y}$ & $\mathrm{Y}$ \\
\hline
\end{tabular}

Table 2.2 - Results of an experiment to determine the efficacy of mark removal by cold acid or alkaline etch treatments.

Note: “ $\mathrm{X}$ ” signifies sample lost during processing

"Y" signifies that defect was seen

"N" signifies that defect was not seen

Prior to processing into EC devices, all chemically etched substrates underwent a final wash in the dishwasher with an alkaline detergent. Upon completion, the samples were subject to a standard coloration test. Table 2.2 shows what defects were seen in the final colored devices.

A number of the substrates in the experimental set were lost in processing due to yield issues such that some correlation data is unavailable. However, significant conclusions can be drawn from the data obtained. To summarize it would seem that the static etching processes have no advantage over simple washing process using DI water and an alkaline detergent. In fact in some instances, the dishwasher process appears to provide better mark removing performance. Several of the intentional defects were affected by the chemical treatments, such as fingerprint, glove marks, and cork spacer marks. Generally only partial removal of the marks was seen. No change in the appearance of the track mark, scratch or metal marking was seen using any of the chosen treatments or by simple washing. Debris from the pilot line sputter coater seems to be linked directly to the likelihood of producing 'shorts' or non-coloring regions in the EC device. Although washing appears to remove such defects, recontamination may occur when loading the substrates into the coater, so general cleanliness and maintenance of the processing equipment is vital in reducing the propensity for this type of defect formation.

The absence of any positive benefit of cold chemical etching of the substrates meant that the approach was abandoned. However, during the investigations of the defect class 
referred to as white dot or non-coloring regions, the use of hot chemical etching as a means of reducing the propensity for white dot formation was investigated. The discussion of white dots is dealt with in Section 2.2.2.2, but the results pertaining to the hot acid or alkali etching are discussed here.

\subsection{Hot acid and hot caustic solutions}

A series of substrate cleaning procedures based on hot caustic and hot acidic baths were evaluated with particular regard to damage thresholds for the coating on the glass, the glass surface and the electrical bus bars that we deposit directly onto the substrate prior to EC device processing. These steps were designed to address the formation of white dots and details of the reasons this approach was adopted are given in Section 2.2.2.2 when the subject of white dots will be dealt with in detail.

Procedures included single and serial cleaning baths, all spanning a range of acid or alkali concentrations, bath temperatures and immersion times. A suitable range of immersion times (of the order of one to several minutes) and cleaning solution concentrations at $50^{\circ} \mathrm{C}$ were found to fall below all of the damage thresholds for substrate structures, while showing indications of excellent cleaning action. In addition, excessive cleaning times developed the otherwise invisible damage features associated with the scribed isolation lines on the substrates, providing a valuable feedback tool for minimizing substrate damage.

As indicated, further discussion of this process will occur in Section 2.2.2.2 where we discuss device defects, particularly shorts and white dots.

\subsubsection{Inspection Methods}

Currently, the thin-film industry does not have a reproducible way to inspect incoming glass lites on-line for defects and contamination that may subsequently lead to nonuniform, streaked, or otherwise defective films. Specific problems may include staining of the glass caused by poor storage conditions, contaminant layers originating from glass handling equipment such as robotic suction cups or cutting wheels and rollers, and airborne organic materials. There is clearly a need to develop a fast but sensitive glass inspection technique, which can be used at various stages of the process to detect sources of contamination, and hence reject the glass before further costly processing is performed.

This section documents the work on development of an inspection system, covering microscopic inspection, laser scanning, different lighting techniques, and water vapor condensation. Further work on defect inspection, in particular the work carried out offline using sophisticated vacuum based analytical equipment is dealt with in Section 2.2.1. This section concentrates on development of processes and procedures that are intended to become part of the routine in-line monitoring process, and are intended to be used to 'weed-out' glass lites which are defective in some way. Section 2.2 deals largely with off-line investigation of defects and their potential causes, and is intended to provide information that will improve the overall process. 


\subsubsection{Development of Optimum Monitoring Technique}

Prior to the beginning of this project, substrate inspection had only been carried out with a microscope or by observation with the naked eye. The microscope was used to look for particle contamination, but obviously limited the area that could be inspected practically. Simple visual observation was adequate to look for gross contamination or marking, but is not a practical long-term solution.

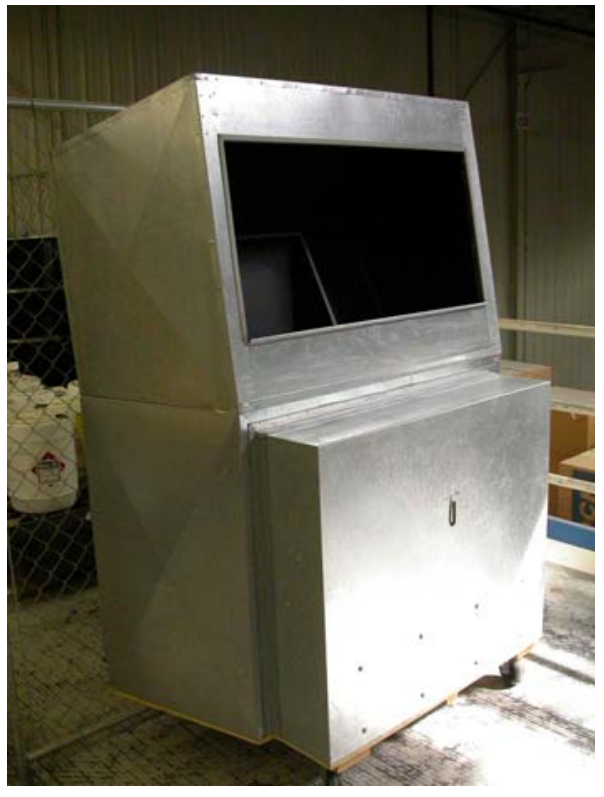

Figure 2.1 - Photograph of glass inspection system.

The process was started by evaluating several different types of lighting for inspection of the glass. These included: front surface diffuse lighting, back lighting, off-angle lighting and edge lighting. All of these methods are routinely used by the glass industry to highlight debris that may be on the surface of the glass. Incidentally, they are also used as an after deposition tool to see defects in sputtered films. However, it was found that these techniques were not effective for many defects, primarily track marks, which may have been on the surface of the substrate.

As a result attention was shifted to an inspection method based on condensing moisture on the surface of the glass. The principle of the technique is based on the fact that water wets different areas of the glass, depending on differences in the surface energy resulting from clean and contaminated regions. The difference in condensation allowed many of the defects to be seen. This was originally done by breathing on the substrates, and indeed this simple, if inefficient, method was used to detect track marks and facilitated removal of around 70\% of the defective glass before it could enter the deposition process.

Based on this work, the two inspection methods — "back lighting ” and "condensation/fog testing "- were combined to develop a more sensitive and reproducible method for detecting substrate marks and defects. Equipment based on this technique has been developed to enable inspection of the SAGE standard large-area substrate size (19” × 37”).

The original design used a manual spray mister, and so complete coverage of a large substrate in one pass was not possible and so the larger substrates had to be examined in segments. The manual spray mister was replaced with a system where the substrate surface was exposed to a high humidity environment. Moisture then condensed onto the substrate surface, and simply varying the humidity within the enclosure could control the 
rate or degree of condensation. A photograph of this updated system is shown in Figure 2.1 .

The inspection system consists of a large enclosure with the inside painted black. Lights are arranged so that they indirectly illuminate the substrate, so any condensation will scatter light towards the observer. The humidity of the enclosure is provided by a heated moisture source via a large bore pipe. A damper allows the level of humidity to be controlled simply by restricting the path for the humid air.

This inspection method is working successfully, and has revealed marks caused by the flat glass washer rollers, scratches and water marks. This method is continuing to be used as a quality screen to remove defective substrates before they enter the deposition process.

\subsubsection{Validation of monitoring techniques}

It is obviously necessary to validate the inspection technique to prove what is detected by the inspection system is actually present on the glass. This was done by fully processing the inspected glass to determine whether defects that had been detected by the inspection system translated into defects on the finished EC device.

\begin{tabular}{|l|l|l|l|l|c|c|c|}
\hline \multirow{2}{*}{$\begin{array}{l}\text { Substrate } \\
\text { marks }\end{array}$} & $\begin{array}{l}\text { Present on } \\
\text { initial } \\
\text { inspection? }\end{array}$ & \multicolumn{5}{|c|}{$\begin{array}{l}\text { Mubstrates marked and then } \\
\text { washed before processing }\end{array}$} & \multicolumn{2}{|c|}{$\begin{array}{l}\text { Substrates marked but not } \\
\text { washed before processing }\end{array}$} \\
\cline { 2 - 7 } & $\mathrm{Y}$ & $\mathrm{X}$ & $\mathrm{N}$ & $\mathrm{N}$ & $\mathrm{Y}$ & $\mathrm{Y}$ & $\mathrm{Y}$ \\
\hline Glove mark & $\mathrm{Y}$ & $\mathrm{X}$ & $\mathrm{N}$ & $\mathrm{N}$ & $\mathrm{Y}$ & $\mathrm{Y}$ & $\mathrm{Y}$ \\
\hline Fingerprint & $\mathrm{Y}$ & $\mathrm{X}$ & $\mathrm{N}$ & $\mathrm{N}$ & $\mathrm{Y}$ & $\mathrm{Y}$ & $\mathrm{Y}$ \\
\hline $\begin{array}{l}\text { Dried water } \\
\text { drop }\end{array}$ & $\mathrm{Y}$ & $\mathrm{X}$ & $\mathrm{Y}$ & $\mathrm{Y}$ & $\mathrm{Y}$ & $\mathrm{Y}$ & $\mathrm{Y}$ \\
\hline Knife scratch & $\mathrm{Y}$ & $\mathrm{X}$ & $\mathrm{N}$ & $\mathrm{N}$ & $\mathrm{Y}$ & $\mathrm{N}$ & $\mathrm{N}$ \\
\hline Coater debris & $\mathrm{Y}$ & $\mathrm{X}$ & $\mathrm{N}$ & $\mathrm{N}$ & $\mathrm{Y}$ & $\mathrm{Y}$ & $\mathrm{Y}$ \\
\hline Suction cup & $\mathrm{Y}$ & $\mathrm{X}$ & $\mathrm{Y}$ & $\mathrm{N}$ & $\mathrm{Y}$ & $\mathrm{Y}$ & $\mathrm{Y}$ \\
\hline Metal mark & $\mathrm{Y}$ & $\mathrm{X}$ & $\mathrm{N}$ & $\mathrm{N}$ & $\mathrm{Y}$ & $\mathrm{Y}$ & $\mathrm{Y}$ \\
\hline Plastic mark & $\mathrm{Y}$ & $\mathrm{X}$ & $\mathrm{N}$ & $\mathrm{N}$ & $\mathrm{Y}$ & $\mathrm{Y}$ & $\mathrm{Y}$ \\
\hline Cork spacer & $\mathrm{Y}$ & $\mathrm{X}$ & $\mathrm{Y}$ & $\mathrm{Y}$ & $\mathrm{Y}$ & $\mathrm{Y}$ & $\mathrm{Y}$ \\
\hline Track mark & & & & & \\
\hline
\end{tabular}

Table 2.3 - Results of an experiment to determine the efficacy of defect detection using our modified fog-box.

Note: “ $\mathrm{X}$ ” signifies sample lost during processing

"Y" signifies that defect was seen

"N" signifies that defect was not seen

This consisted of carrying out a substrate marking experiment similar to that described in the chemical etching section (Section 2.1.1.2 above). Furthermore, full validation of the technique was begun by implementing it as part of the full SageGlass ${ }^{\circledR}$ process. An experiment was carried out where substrates with suspected track marks and additional added defects were inspected and then processed into devices. The purpose of the experiment was, first, to determine whether the inspection technique could detect known 
marks on substrates; second, to correlate the marks seen during inspection with final device defects; and third, to understand the impact of washing on mark removal. Six substrates were washed, inspected, marked, and re-inspected. Three of the samples were then processed into EC devices. The other three samples were re-washed and then processed into EC devices. After processing, each EC device was switched and a photograph was taken for final defect characterization. Table 2.3 summarizes the appearance or absence of defects in final EC devices.

To summarize the results:

- Correlation of all defects that were intentionally placed on the substrates was 100\% using the updated condensation/backlighting method.

- All marks identified by the inspection technique, except sprinkled debris taken from the coating chamber, were present on all of the devices post EC device processing. It is likely that the debris from the chamber was removed from the surface before processing by the air blow-off steps, which are routinely implemented prior to first-layer deposition.

- Post-washing inspection indicated that several of the defects had been either totally or partially removed. Partially removed defects were less well defined when viewed using the inspection system. The absence of the defect in the inspection step correlated with the absence in the final device.

- As expected, washing had little effect on the appearance of the track mark, scratches or metal marking; these marks were clearly visible both in post wash inspection and in the final device.

The next stage in the validation of the technique was to implement it as part of the SageGlass ${ }^{\circledR}$ process itself. As detailed above, the technique is now routinely used to screen substrates before thin-film deposition. The substrate tracking systemhas also been improved to provide better comparisons between what is observed during the inspection and what is observed on the finished devices. Records are kept for every substrate describing observable "defects" at inspection and in the final EC device. In this way a database is being built, which allows correlation to be made between defects visible at inspection with those present on the final device. This effort also enables continual updating of the list of known problem defects, which improves the screening process, resulting in higher product yields.

It has been found that the inspection system shows a multitude of "artifacts" on each substrate. While some appear to impact final device quality, a significant proportion of these are not present in the final device. It is clearly important to differentiate between the two types of artifact. Indeed, progress has already been made in this area, and key "artifacts" which impact the quality of the final device have been identified. These defects are primarily associated with marks on the glass derived from the manufacturing process (track marks), fingerprints, scratches and pinholes. 
Overall, the validation of the inspection techniques leads to a high degree of confidence that many substrate defects can be detected prior to EC device fabrication.

Differentiation between genuine defects, and artifacts that show up during inspection, but are not present in the finished EC device, has also been possible.

\subsubsection{Glass Supplier Quality}

The previous two sections (2.1.1 and 2.1.2) have discussed various aspects of glass cleaning and inspection, aimed at minimization of defects seen in EC devices due to substrate imperfections. Clearly, the as-received quality of the glass is a vital part of ensuring the highest quality for subsequent processes. It is therefore critical to try to establish a good relationship with the glass supplier to help develop and control the procedures that will minimize potential problems. This section discusses the work done jointly between SAGE and the glass supplier in order to accomplish this.

Prior to the beginning of this project, one of the problems that led to yield loss was the appearance of thin (approximately $4 \mathrm{~mm}$ wide) lines across the full width of the device that would color at a different rate to the rest of the device. These are referred to as track marks. By monitoring the position of the glass substrates on the float line, it was apparent that track marks were coming from wheels that were touching the top surface of the glass. After discussions with the glass supplier, a procedure was implemented to minimize the number of such wheels in contact with the glass, thus basically eliminating the occurrence of track mark defects.

After this initial success, all steps of the glass manufacturing and coating process were jointly scrutinized by SAGE and the glass manufacturer, in order to produce a process which would yield high quality glass, suitable for EC device manufacture. This collaboration resulted in a written procedure for producing the glass, which encompassed all stages of the production process, and in particular: coating, cutting, packing and shipping. An abbreviated version of this procedure is given in Appendix 1.

A further example of the collaboration between SAGE and the glass manufacturer concerned the issue of robotic suction cup markers that were showing up in finished EC devices. The solution to this problem was to cover the suction cups with a surgical 'boot' to prevent them from coming into direct contact with the glass surface. This appeared to alleviate the problem of the suction cup marks.

A further complication occurred when the manufacturer of the coated glass moved the operation to a different facility. A close collaboration with the quality team there was established, but due to differences in the production procedures and hardware, it was not possible to transfer the process exactly. In particular, the new facility could not cut the smaller sizes that had been obtained from the first production plant. Different cutting approaches were therefore investigated: on-line cutting of larger pieces $(4 \mathrm{ft} \times 3 \mathrm{ft}$ approximately) which would then be cut down to size at SAGE; and two off-line cutting approaches, one slightly more automated than the other. It was found that the substrates which were cut off-line showed significant substrate quality issues, including scratches, 
chips, cutting marks and oil stains. It was therefore decided to have the glass cut on-line to the slightly larger size and then cut it at SAGE to the final sizes.

In addition, upon moving the production to the second plant, it was found that the lack of the interleaving material, which had been removed after the determination that it was leading to large area patterning of the devices, was causing an increase in the number of suction cup marks from the packing robots. After a further meeting of the SAGE team with the supplier quality personnel, a plan was developed to minimize suction cup marking. This plan included the use of a new interleaving material, and this was found to reduce the problems that had been seen to an acceptable level.

All in all, a very productive relationship with the glass supplier has been developed. The most tangible result of this is a production checklist, but significant knowledge that will allow us to more easily go through the process when qualifying a second supplier has been gained.

\subsubsection{Process Quality Procedure}

Three aspects of substrate preparation have so far been discussed: substrate cleaning, substrate inspection, and the relationship with the glass supplier. All the work discussed has one aim: to produce the highest quality substrates for deposition of EC devices. It should therefore be clear that the substrate preparation and handling is of crucial importance to the final quality of the finished EC devices. For this reason, and as part of the company-wide quality initiative, a review of the substrate preparation process - from the substrate fabrication right up to the point at which the EC films were deposited - was undertaken. This involved several different quality initiatives, some of which are discussed below.

The substrate preparation process involves a number of different steps. Very briefly, the steps consist of the following:

- Receipt, unpacking and storage of glass;

- Cutting to size;

- Line isolation;

- Seaming (Removal of the sharp edges);

- Initial wash;

- Bus bar application;

- Transportation to tempering facility;

- Tempering;

- Return from tempering facility;

- Storage; 
- Final wash.

Each of these steps has been analyzed with a view to quality improvement, with four main areas of focus in mind: time savings, process improvements, training and standardization. A more complete list of these improvements can be found in Appendix 2, but some highlights are given here as illustrative examples of the kind of improvements that are being made throughout the process. These are discussed in six general areas.

\subsubsection{Glass Cutting/Handling}

In the areas of glass cutting and handling, numerous improvements have been made throughout the duration of the project. These include the manufacture of a new loading table for the glass cutter which was designed so that there is less possibility of damaging the surface of the glass during cutting. Micrometer stops were also added to the glass cutting table to make it easier to cut the correct sizes accurately and repeatably.

The manufacture of new transport carts for transportation of or standard sizes of glass was also initiated. These are now used to transport the glass to and from the tempering operation. This involves two journeys by truck, and so the design of the boxes needs to be capable of holding a number of glass substrates securely, so that the possibility of breakage is completely eliminated. These boxes have been fabricated and work very well.

In addition, modifications have been made to the existing carts to allow for the transportation and storage of substrates of irregular dimensions, such as would be required for automotive windshield shapes.

\subsubsection{Substrate Patterning}

It is necessary to pattern the transparent conductor (TC). Prior to the beginning of this project, a great deal of effort was spent modifying the equipment to produce a repeatable pattern. This work has continued, and the equipment has been redesigned to allow the addition of a bar which acts as a physical stop to ensure the distance from the edge of the glass to the pattern is the same each time. Frequent measurement, along with statistical process control (SPC) techniques - discussed later - and physical adjustments whenever necessary, ensure that the quality of the process positioning relative to the edge of the glass is maintained.

\subsubsection{Screen Printing}

Currently, a screen-printing technique is used to apply the bus-bars to the substrates. This step is of critical importance, as any small particles of stray material (down to sizes comparable with the thickness of the thin films) that get onto the active area will lead to a short. Shorts are obviously unacceptable, and so a significant amount of effort has been expended on this process to try to keep it as clean as possible. 
There have been two general areas that have been addressed intensively during this project. The first one was the location of the substrate when loaded into the screenprinter. The consistency of this step ensures the bus-bars are placed as repeatably as possible, and is a major help in simplifying further downstream processes. Installation of a substantially modified substrate table assembly has recently been completed, allowing rapid, accurate loading of the substrates prior to printing.

The second area addressed was screen cleaning. This is important, because after using the screen, the excess conductive material must be removed, because as has been said, any stray material on the active area will lead to unacceptable device defects. Cross contamination from an inadequately cleaned screen can easily occur. Some improvements have been made to the cleaning materials used, and this has led to an overall improvement in cleanliness, clearly a desirable outcome.

\subsubsection{General Quality improvement}

One of the currently popular quality improvement techniques within lean manufacturing is known as kaizen. This is a technique for reducing waste within the manufacturing environment, and uses several simple tools to improve quality and efficiency. One of these is known as 5S, where the 'S's stand for:

- Sorting - what is needed right now and what is not;

- Straightening - a place for everything and everything in its place;

- Sweep - is both physical and visual, removing any abnormalities, sources of backsliding, clutter, or poor visibility;

- Standardize - by establishing “what good looks like” and rules for upkeep;

- Sustain - establish procedures to maintain things in place.

Kaizen includes making the manufacturing process simpler with carefully designed workcells, in which the work-pieces travel a minimum distance, immediately traveling from the output of one operation to the input of the next. As part of the quality improvement procedure, work-cell design was implemented and 5S carried out on the substrate preparation process. Some of the direct benefits are listed below:

- Improved the workflow (including continuous operation from glass pallet through isolation line fabrication) during the entire preparation process;

- Establishment of a dedicated cabinet for tools storage in the substrate preparation work area;

- Improvement of the lighting for the inspection area in the glass washroom;

- Scheduled periodic internal process audits for the substrate preparation area;

- Established preventative maintenance (PM) schedule for the sandblaster. 
Furthermore, at the same time requirements for the process from the perspective of the next step of EC device manufacture were examined. This required that certain critical dimensions, such as glass size and position of line isolation and bus-bars were measured. Equipment was procured for precision measurements and to enable SPC techniques to be used. Some of these measurements and the documentation efforts to support the quality process are discussed briefly in the following two sections.

\subsubsection{Metrology/SPC}

Statistical Process Control (SPC) methods ${ }^{7}$ are useful tools to monitor the results of a manufacturing process. However, before they can be implemented, the measurement precision must be high enough to allow the analysis of the results to be meaningful. In the substrate preparation process we are most interested in using these techniques to monitor (a) the size of the substrates after they are cut; (b) the placement of the bus-bars and line isolation relative to the edge of the glass; and (c) the level of stress in the glass after tempering. It was therefore necessary to obtain measurement equipment of sufficient precision to allow measurements of all these parameters to be made. To that end the following were obtained:

- A long-range caliper that allows measurement of the dimensions of large sheets of glass with a precision of 0.001 ”. This is a dramatic improvement over the previous technique which allowed measurement precision of $\pm 1 / 16$ ” (0.0625”);

- A Glancing Angle Surface Polarimetry (GASP) system to measure the surface compressive stresses in the tempered glass pieces. This is used to measure variation within a lite, lite-to-lite variation within a load, and variation from run-to-run;

- A traveling microscope for isolation line and bus-bar placement, which was manufactured in-house.

Two example SPC charts showing the measurements for the lengths and the widths of one particular size of cut substrates are shown in Appendix 3. It is immediately clear that the measurement precision has been dramatically improved from the 0.0625 ” that we had been the norm prior to the introduction of these SPC techniques.

\subsubsection{Documentation}

Also as part of the on-going quality improvement program, a good deal has been invested in documenting the processes and procedures. A new system for documenting all

\footnotetext{
7 ‘Understanding Statistical Process Control', D.J.Wheeler and D.S.Chambers, SPC Press (1992)
} 
substrate preparation operations consisting of a central file with links to various other data files has been constructed, and includes:

- Links to the different tempering batches;

- Measurements, settings, and PM operations;

- Manufacturer’s glass lot information, with batch conformance data.

Individual process sheets in binders conveniently located at individual workstations are also available. This information is currently in paper form, but will later be transferred to an electronic format to allow searching, documenting, tracking, etc.

As with all documentation processes, the process requires that the documents are maintained in an up-to-date state, and this requires continual monitoring and auditing. We are confident that we have instituted procedures that will maintain the documentation as required for it to be useful.

\subsubsection{Summary of Task 1}

Task 1 began with the ambitious goal of automatically monitoring glass cleanliness down to the scale of very small particles, thus reducing the impact of such particles and other contaminants on the quality of finished EC devices. However, it has been found that in order to maximize the quality of the substrate preparation steps, the problem can be broken into two parts. First, an inspection method has been developed to detect 'large scale' defects. 'Large scale' means anything which is visible without the aid of a microscope, which in practice could mean anything down to about $0.5 \mathrm{~mm}$. This is the substrate inspection station, and is discussed in some detail in Section 2.1.2. Next, techniques aimed at reducing the number of particles generated throughout the process have been set in place. The inspection techniques have identified the types and sizes of defects that are likely to cause rejections later in the process, and now there is a method of substrate inspection allowing rejection of the vast majority of the contaminated samples before they enter the process. This has resulted in very low losses from substrate contamination defects.

The second product of this task has been a substrate preparation quality procedure, and as with all quality processes, this is continually evolving and improving. However, there are some indications of success in this area in the decline of measured rates for two of the most troublesome defects, shorts and white dots. This has been done by using both microscopic and macroscopic inspection techniques, in order to study the effectiveness of the cleaning methods, and using the results from these measurements to improve those methods. We have also worked closely with the glass supplier to reduce or eliminate many of the defects resulting directly from the float glass process.

During this project, significant progress has been made on the extremely important issue of substrate inspection and cleaning. This has been a wide-ranging area, encompassing installation and maintenance of the flat glass washer, chemical cleaning, detergent 
experiments and inspection techniques. In addition, an extremely good working relationship has been forged with the glass supplier. Finally, we have embarked upon the implementation of a quality procedure incorporating all the elements required for qualifying the substrate preparation process for scale up to the next stage of manufacture.

\subsection{Task 2 - Identification and Minimization of Particle Generation Sources}

As is well known throughout the thin film industry, particulate contamination can cause significant problems. This is particularly true for electrochromics, where the effects of very small defects can be magnified many times. There are several sources of potential particulate contamination within the process, and this section deals with the identification and minimization of particles generated by these sources. The nature of the defects caused by these particles is discussed, along with the work that has been done to identify the root cause of the contamination from an analytical perspective, and the measures that have been taken to eliminate or reduce their impact on the process.

The process can be broken in three areas: substrate preparation, coating, and heating. The discussion of the effects that the particles can have on EC devices is applicable to any particulate source: the exact nature of the defect will depend on the location within the process and consequently the location within the EC stack, and the physical characteristics of the particle itself, i.e. size, electrical conductivity, chemical reactivity, etc.

The substrate preparation step has been discussed in some detail in Section 2.1. However, particle generation sources themselves were not explicitly discussed. Preparation of the substrate involves several individual steps that are prone to particle generation and were not discussed previously: cutting, edge-seaming, line isolation, busbar application, transportation, and tempering.

During cutting, the glass is scored along the desired line, and then snapped along the score line. This has the effect of microscopically 'exploding' the surface of the glass along the cut, yielding many small glass fragments, some of which may be coated with conductive tin oxide. These particles may be loosely adhered to the surface, or may build-up on the cutting equipment and contaminate subsequently processed substrates.

To prevent glass from breaking during tempering or during the heating steps of the EC device fabrication process, it has been found necessary to seam the edges of the glass. This process also removes the sharp corners from the cut edges by grinding, which also makes handling the glass much safer. A free-standing dual belt sander is used for this purpose. This grinding operation is an obvious source of particle contamination, and extreme care is taken to ensure that substrates are immediately rinsed with DI water prior to washing, in order to remove as much contamination as possible, and thereby prevent scratching of the surfaces by abrasive material and glass chips.

Next, the coating on the surface of the substrate has to be patterned. Currently, a sand abrasion technique is used to form the pattern. In this process, the conductive coating is 
blasted off the glass using a well-controlled stream of abrasive particles. Clearly, both the abrasive as well as the abraded material are sources of particles.

The final preparation step before tempering is bus-bar application. A modified screenprinting technique is used to apply conductive bus-bars to the substrate. During bus-bar application, it is always possible for flakes of conductive material to find their way into the hardware of the applicator, which can be stirred up and later redeposit onto the active area of the substrates. This really is a general issue with workplace cleanliness, but the EC device process is particularly sensitive to conductive particles, as they can cause shorts, which are very clearly visible when devices are switching or in the fully colored state.

Similarly, during transportation to the off-site tempering operation, it is necessary to pack the glass into specially designed transportation boxes. Once again, the boxes can become a trap for particles - such as glass fragments from cutting, or conductive particles from bus-bar application - that may have been loosely adhered to the substrates, but 'knockedoff' by the vibration experienced during the journey. This debris can be stirred up and redeposit on the active area of the devices. This is particularly important immediately prior to tempering, as anything that gets onto the glass at this time will be 'fired-into' the surface, and become almost impossible to remove. Obviously, then, anything that contaminates the glass surface during the tempering operation, i.e. after removal from the transportation box, loading into the tempering furnace, and the actual tempering operation, will lead to contamination that will be fired into the surface of the glass, leading to defects in the finished EC devices.

Several potential contamination sources throughout the EC device process have been described. Many of these sources are identical for standard coated glass products. However, unlike in static coatings, it is noted that the effects of particles on EC devices can be much larger than the size of the particle itself, making EC devices a 'worst case scenario' for defects. There now follows a brief explanation of how very small particles can lead to large defects in EC devices.

When incorporated into the films, even very small particles can have a direct impact on product quality, leading to defects or discontinuities in the coatings. For static films, voids are only really significant when they are big enough to be seen, and also give a high optical contrast with the surrounding films. On the other hand, in the case of electrochromic (EC) devices, particle contamination is most likely to produce visible pinholes (non-coloring areas) or as noted earlier, shorts. Due to the high contrast ratio between the colored and clear states of the EC device, non-coloring areas are highly visible in EC devices. The areas that are affected are often significantly larger than the particle itself. The reason for this is not immediately obvious and will be dealt with in Section 2.2.2.2. However, in the case of a short, the effect can be understood by consideration of the voltage distribution around the short when compared to an unshorted region.

For an ideal unshorted EC device at equilibrium in the colored state, the voltage between the transparent conductors at any point on the device will be equal to the supply voltage, 
i.e. one of the transparent conductors will be at the positive supply voltage and the other transparent conductor will be at the negative supply voltage. In a real all solid state EC device, there will be some current 'leaking' through the device, leading to small deviations from this ideal picture, but these are not relevant to this discussion. Note that a short will not be visible when the device is in the bleached state. If we make the reasonable assumption ${ }^{8}$ that the depth of coloration, or optical density (OD), will be directly proportional to the voltage between the transparent conductors (TCs) at the point of interest, we can see that the coloration of the ideal device will be uniform. However, if we introduce a short - a direct connection between the TCs - then the voltage between the TCs at that point will be zero. This means that there will be no coloration at the position of the short. In addition, the current flow locally will be radially towards the short in one conductor and radially away from it in the other, leading to a radial potential drop in both TCs. The voltage some large distance away from the short, perhaps as much as $10 \mathrm{~mm}$ - determined by the sheet resistance and the current flowing through the short will be equal to the supply voltage, and will gradually drop to zero as the short is approached. The depth of coloration will therefore follow this voltage profile, leading to an affected area that is significantly larger than the size of the short itself. This means that subtle imperfections, which may be acceptable for static glazings, can become highlighted and lead to much larger defects when a dynamic window changes state.

It is important to understand and control all of the origins of particle contamination, and to minimize each one. This is particularly true for EC window fabrication, and the goal is therefore to track down and minimize all of the possible sources of contamination, from receipt of glass, all the way through the process to the finished product. Previous Task 1 discussions concentrated on control of the general cleanliness of the substrate. The following sections deal with the efforts at control and minimization of particles in the vacuum system and associated processes.

\subsubsection{Identification and Minimization}

This section focuses on identification and minimization of sources of particulate contamination. We start by examining the sputter coater, and then move on to look at other sources within the process. This section addresses the effects of the particles on the performance of the EC device, and details the understanding of the microscopic mechanisms of defect formation.

The following discussion is broken into three main areas: first the identification methods that have been developed at various stages of the process and then some of the attempts that have been made to minimize the number of particles incident on the substrate during

\footnotetext{
${ }^{8}$ In EC devices, it is found that the depth of coloration is indeed a strong function of the applied voltage, although it is not perfectly linear. For example, application of zero volts does not cause any coloration, and so the device remains in the equilibrium state, which in this case is fully bleached. Application of small voltages will lead to small color changes, up to a point where full coloration is achieved. This assumption is therefore very reasonable for a very wide range of coloration states.
} 
the process are addressed. Third, the detective work done to understand the particle types and their effects on the finished product are examined.

\subsubsection{Particles in the Sputtering Process}

Particulates are implicated in a number of optical thin film defects that may limit pilot line yield. The entire EC device manufacturing process has been examined for potential sources of particulate contamination. Initially particles generated in the vacuum coatercoater dust - are the focus of attention.

During the sputtering process, material is not just deposited onto the intended substrate, but also all the other line-of-sight surfaces inside the process chamber. These films will continue to build-up until they eventually flake off, due to the accumulation of sufficient stress. Particle generation is likely during heating and cooling operations, as well as operations where the stresses on the vacuum chamber walls are rapidly changed, for example venting and pump-down, or where a roller travels over a coated region and grinds off the underlying films. This generation mechanism can be said to lead to mechanically generated particles.

Particles can also be generated when a sputtering target undergoes an arcing event. This can happen with any type of target, but is more probable when the sputtered layer is a non-conductive dielectric layer. During the sputtering process, the deposition of nonconductive layers on surfaces near to, or in, the plasma, can lead to significant charge build-up, and eventually, when the local field is high enough to cause dielectric breakdown, a sudden and destructive discharge (or arc) can occur. This can obviously lead to particles and molten discharge products, as there is an event similar to a miniature lightning strike within the chamber. We will refer to this generation mechanism as leading to arc generated particles.

The material that flakes off in either of these cases, can lead to particles with a wide variation in sizes, and these particles can contaminate the entire vacuum system, even affecting subsequent coating operations. Any particles finding their way onto the substrate can then be overcoated by subsequently deposited films, possibly leading to shorts if the particles themselves are conductive. In some cases, these can be brushed off during washing or 'popped-off' during a temperature excursion, leaving a pinhole or void. In the case of EC devices, this gives the possibility of a direct connection between the top and bottom conductive layers, which can lead to a short.

Transport through the coater is currently the rate-determining step for the EC device manufacturing process. The heating and deposition equipment can be cleaned and maintained without necessarily affecting throughput, and so have little impact on the cost of the process. Therefore, in addition to particles having an impact on the coating quality, the downtime for maintenance of the sputter coater can have a significant impact on product throughput and therefore product unit cost. Any methods that can reduce the generation of particles in the coater, and therefore allow longer periods between maintenance events, can give a significant throughput improvement, reduction in reject 


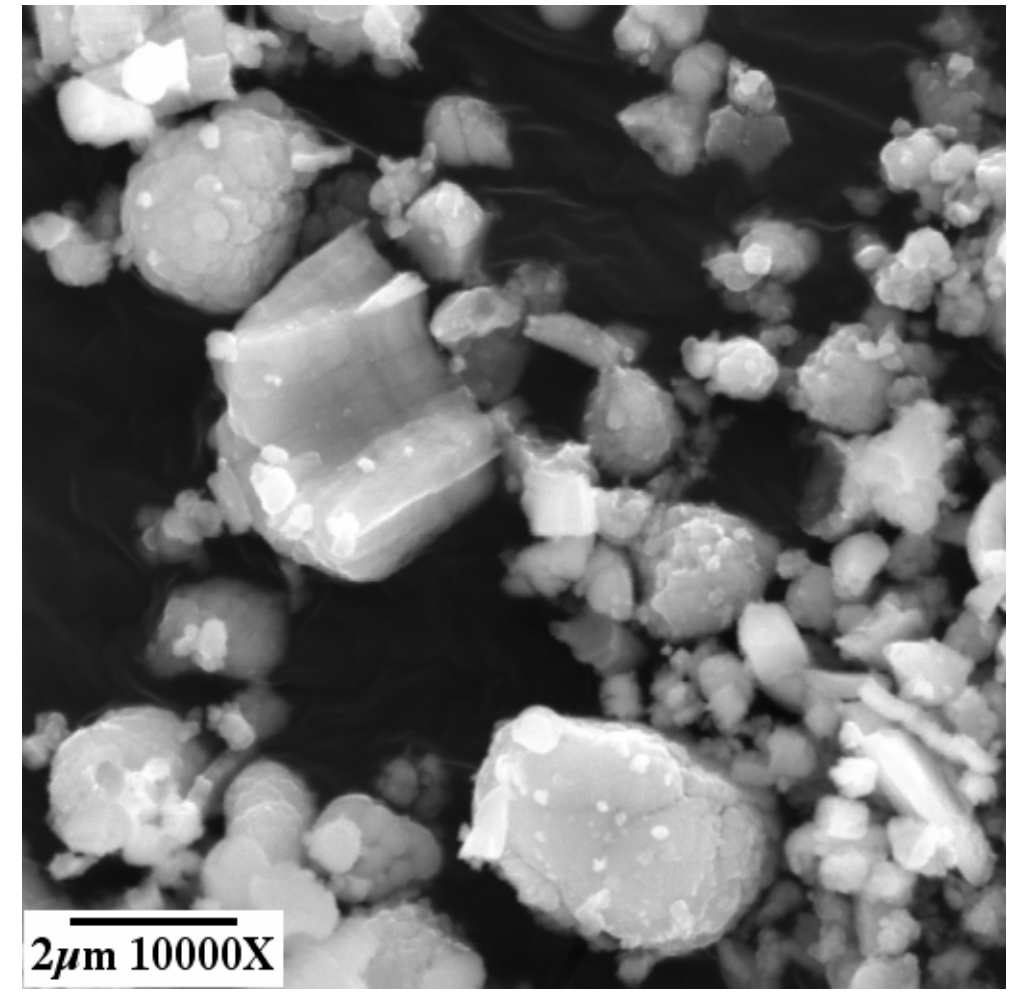

Figure 2.2 - Scanning electron micrograph of coater dust. The smallest resolved particle is less than $40 \mathrm{~nm}$ in diameter. rates and therefore improvement in product quality. For these reasons, a significant effort has been expended on addressing the issue of particles in the coater.

Three approaches were taken to characterize particulate contamination, two general approaches, and one that is particular to the coating process. In one of the general analysis approaches, samples from representative surfaces were submitted for metals analysis by inductively coupled plasma mass spectrometry (ICP MS). In the other, samples were analyzed by scanning electron

microscopy - energy dispersive x-ray analysis (SEM - EDS). We used kapton tape to trap the particles, and swabs were taken from representative areas when the coater was vented for routine maintenance. Finally in the approach intended to look specifically at particles generated during the sputtering process, substrates were immersed in a solvent bath, which was subsequently filtered to capture dislodged coater dust.

Samples taken from the coater during a routine vent were analyzed using SEM/EDS. The detailed analysis of the results is given in Appendix 4. As an example of the results, we found that the dust in the load-lock of the sputter coater contained all the elements of the sputter deposited EC films.

The scanning electron micrograph in Figure 2.2 provides a representative picture of the size and morphology of coater dust swabbed from a substrate carrier. In general, the particles ranged from tens of nanometers to several microns in diameter. The larger particles displayed a stacked film structure, whereas the smaller particles appeared platelike. It is likely that these particles originated from continuously deposited films that were ground up by the mechanical action of the transport mechanism in the vacuum coater. SEM-EDS analysis revealed that the elemental composition of the particles is consistent with the elements that comprise the vacuum deposited material. It was found 
that when the substrate carrier travels through the coater, mechanical contact grinds off accumulated coating.

ICP-MS analysis revealed that the coater dust consisted of material from all of the vacuum deposition processes, including those invisible to SEM-EDS (elements lighter than sodium). Consequently, none of the vacuum deposition processes could be ruled out as a source of material for coater dust. This finding is consistent with dust originating from a continuous coating that has accumulated on the substrate carrier and is subsequently ground off by mechanical contact. It was also found that coater dust of similar composition penetrated into the remote antechambers of the vacuum coater. This probably results from turbulent, viscous flow, during venting, or possibly from electrostatic deposition.

As discussed, the major problem with the sputtering process regarding particulate contamination is that the particles are generated within the vacuum system itself. At the beginning of the project, the routine maintenance schedule for the coater consisted of sandblasting the removable parts of the vacuum chamber and hand abrading the parts that could not easily be removed, to clean off the build-up of thin films. The timing for such maintenance events was based upon previous empirical experience with a margin for 'safety'. Typically, the downtime caused by these events represented $10-15 \%$ of the available operating time for the coater. Combining this with other unforeseen incidents lead to an overall downtime for the coater of around $20-25 \%$.

Since instigating the measures described in this report, the unscheduled downtime has been dramatically reduced as can be seen in Figure 2.3. 


\section{Coater Run Time vs Down Time Analysis}

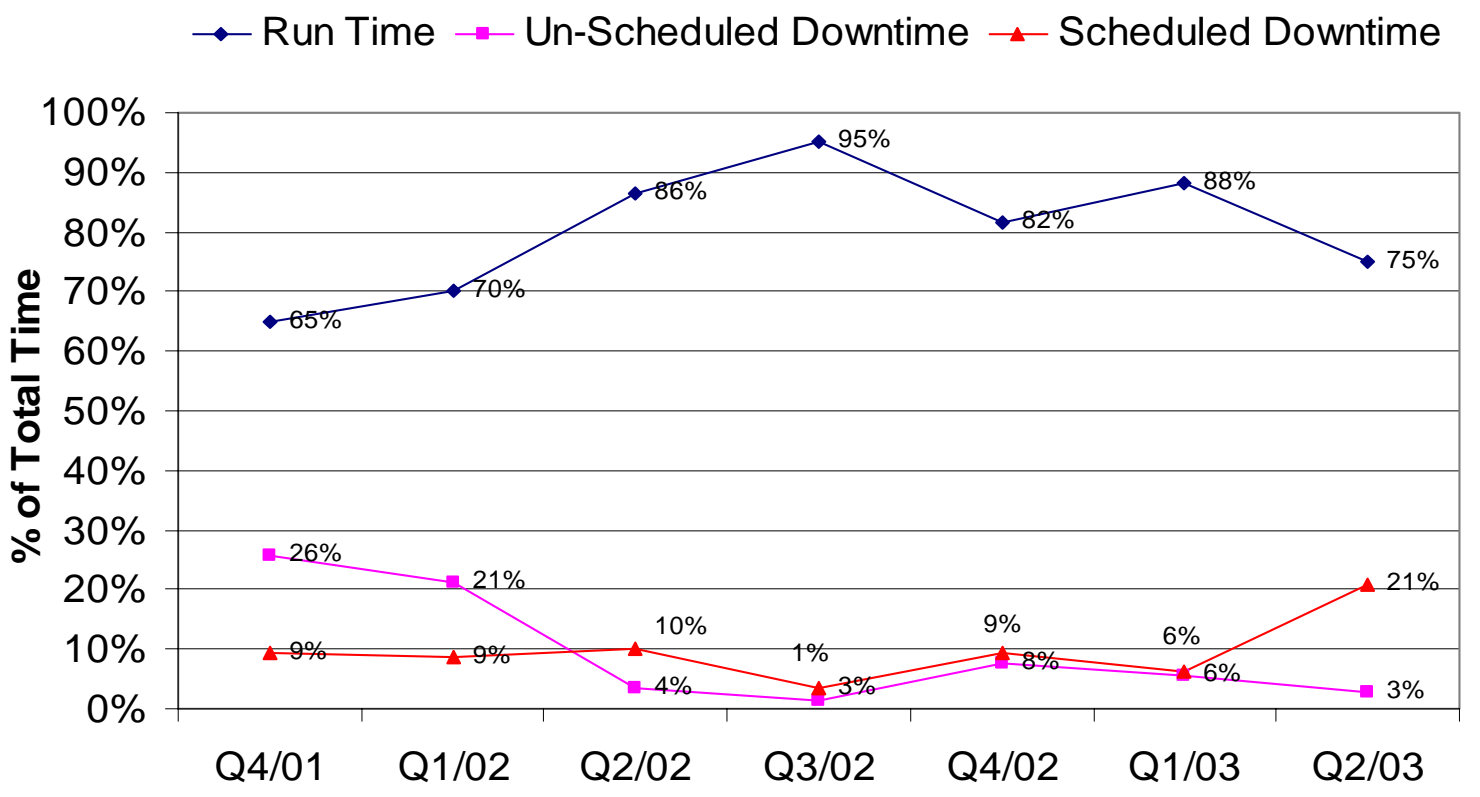

Figure 2.3 - Plot of the percentage run time, scheduled and un-scheduled downtime for the pilot line coater. Unscheduled downtime has been reduced from around $25 \%$ at the start of the project to approximately $3 \%$ at the time of writing.

Several improvements have been made to the coater in order to reduce particulate contamination. A brief explanation of some of these improvements and their impact on the process follows.

As indicated earlier, most of the deposition shielding in the coater was not easily removable. Two complete sets of thin gauge stainless steel (SS) shields were therefore fabricated and installed. These replaced the copper tubs that are more porous and very heavy. Using SS reduces the adsorption of atmospheric gases during the time when the coater is vented, and thereby reduces any contamination by prolonged outgassing from the material and trapped gasses in the construction. The lighter weight makes it easier to remove and clean the shielding, which has the additional benefit of making it easier to schedule cleaning, as there is now no requirement for the use of a crane, and this has the effect of increasing the frequency of cleaning. The second set of shielding reduces the downtime and also the exposure to air, because cleaning does not have to take place prior to re-installation of the same shielding.

A second area of improvement is related to the target bonding. For one target, contamination by the bonding agent was prevalent when the target arced. In order to preclude this, constructive exchanges with the manufacturer resulted in a manufacturing specification for the use of tape in the butt joints where the target material is bonded to 
the backing plate. Subsequent testing shows this to be $100 \%$ effective at suppressing the contamination at current power levels. Further details regarding this process are covered in Section 2.2.2.1.

Another significant source of contamination resulted from arcing of one of the other cathodes. In addition to producing contamination, this can cause small process instabilities. After noting a possible site for this, an insulating silicon annulus was made and installed. Arcing was notably attenuated, inherently reducing the opportunity for contamination caused by this event. Line shorts (which are discussed later) are a similar type of contamination attributed to arcing, and from the work done monitoring this type of defect, it is concluded that installation of the annulus has caused a significant reduction in this type of short. We plan to further increase the size of the annulus to see if additional benefit can be gained.

There is some belief that contamination is occurring in the load lock during pump down and venting. In that regard we have instituted the following:

- Slower pump down times and vent times to minimize turbulence;

- The vent gas was originally directed straight into the side of the chamber. A diffusion plate was added to redirect and reduce the flow during venting;

- The roughing port was also on the side of the load-lock. To minimize the flow of dust across the glass during pump-down the roughing port was moved to the center of the chamber bottom;

- More frequent cleanings were instituted.

In addition, we have begun a rigorous regime of more frequent glass carrier cleaning.

The number of occurrences of each defect is closely monitored, and analyzed using Pareto methods. Reduction in the observations of each of the defects discussed above has been seen. This monitoring will continue in the future, and will enable further reductions in the incidence of defects in the EC device manufacturing process.

\subsubsection{Particle Reduction during Substrate Preparation}

After the glass is received, there are several operations that must be carried out prior to deposition of the EC device films. These have been discussed in some detail in Section 2.1.4. However, to summarize, the sample is first cut to size, and then the sharp corners left by the cutting are removed. Next, the TC is patterned, prior to the glass being washed and passed onto the bus-bar operation.

Each of these steps is prone to generation of particles, which must be removed before the substrates are passed onto the next stage of the process. It should be noted that the substrate preparation process concludes with a final wash, at which point most if not all of the particles should be removed. However, we are not only concerned with the particles themselves (as they can lead to defects such as shorts or non-coloring regions), 
but also with the damage that the particles can do to the glass surface. During cutting, a sharp wheel is rolled across the surface of the glass, to create a 'score' mark in the surface. This process effectively crushes the glass directly beneath the roller, and flakes pieces of it in the immediate vicinity of the score. This score then acts as a guide or seed for a crack to propagate through the glass as it is snapped. Both of these activities can lead to the generation of small fragments of glass, and this can be seen in Figure 2.4, where we see a close-up view of a score mark prior to breaking.

The glass is then broken into two pieces simply by snapping using the score as a guide

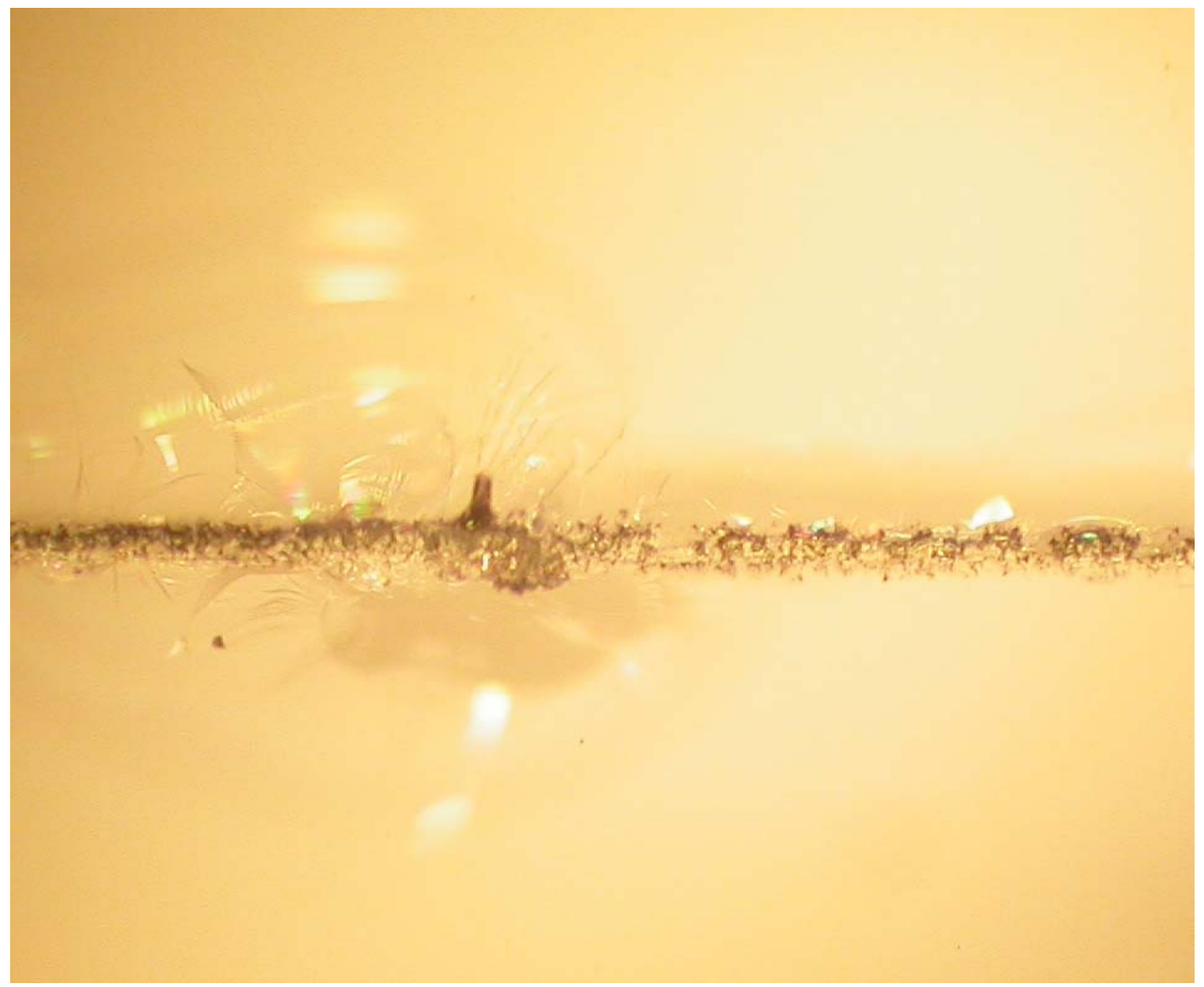

Figure 2.4 - Close-up view of a score mark in glass prior to breaking, showing significant glass damage and some loose particulate contamination. Many particles are visible near to the score, and some flakes where the glass has been removed are visible, particularly over to the left of the image.

for the crack to propagate. The debris from the score is then flung away from the crack, and is redistributed across a larger area of the glass substrate, as well as settling onto the cutting equipment, from where it can contaminate other glass pieces. 
To address this issue, a standard process for cleaning the cutting equipment prior to use has been established. This encompasses cleaning and set-up of the equipment, and is part of the overall quality improvement process. Although there has been no quantitative measurement in the reduction in contamination as a result of these remedial measures, it is clear that cleaning the cutting equipment each time it is used has made a significant improvement to the cleanliness of the process.

After cutting, the sharp edges of the glass are then ground off in a process known as seaming. Obviously, a grinding operation can generate a significant quantity of particles, both from the glass being removed, and the abrasive material being used to remove it. In order to help reduce the number of particles finding their way onto the substrate (to mitigate film damage) a vacuum system to remove the debris from the grinding operation before it can get onto the glass surface has been installed. A procedure to clean the equipment prior to each use has also been instituted, and clearly defined process procedures and a preventative maintenance (PM) schedule have also been set up. The PM procedure involves cleaning the equipment thoroughly, changing the abrasive belts, and emptying the vacuum system.
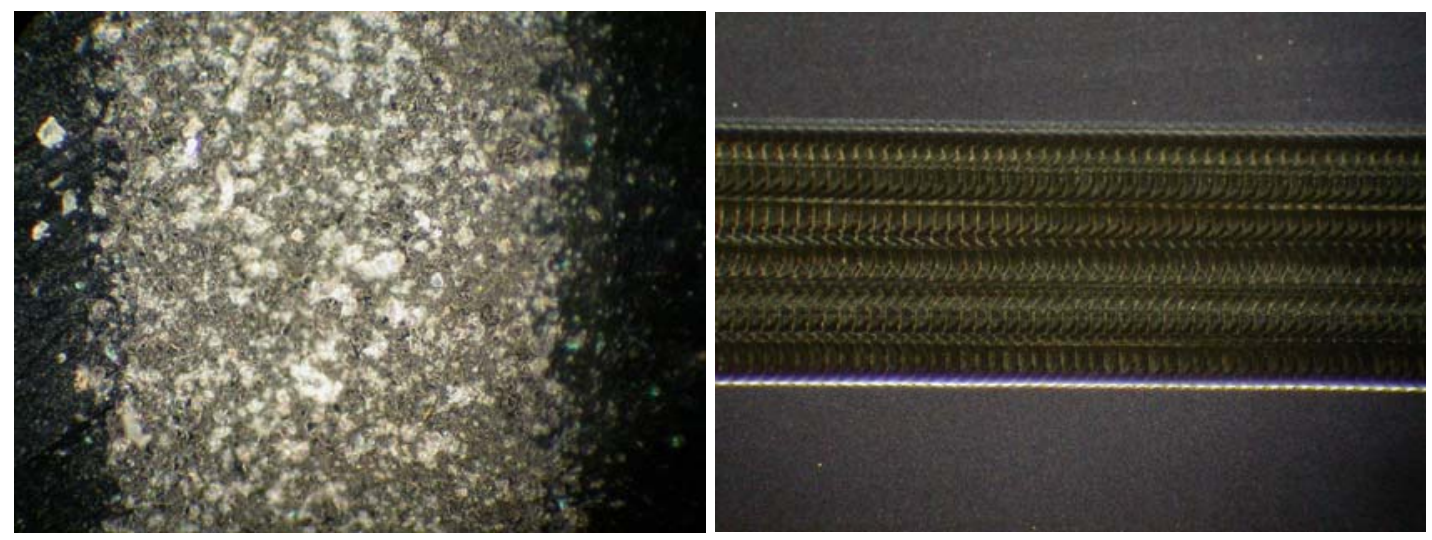

Figure 2.5 - Examples of dark field images of sandblasted, running vertically (on the left - 100x magnification) and laser isolated, running horizontally (right - 200x magnification) lines.

After cutting and seaming, the glass is patterned. This is done by sandblasting using a homemade microblaster. The substrate is placed on a movable flat bed, and is moved through the sandblasting nozzle. The abrasive media are directed at an angle of roughly $45^{\circ}$ to the surface normal, to ensure that they are predominantly directed away from the active surface. A modified vacuum cleaner is used to suck the media away from the delivery system, again in an effort to remove the majority of the particles from the active area.

The sandblasting operation is intrinsically dirty, and so different possibilities for producing the pattern have been considered. One of the approaches is laser ablation of the TC. Excellent isolation can be achieved using commercially available lasers, but the 
cost of the laser system is high. Samples can be sent out to have the patterning applied by a third party, but keeping the glass clean during shipping and processing is a significant challenge because of the additional handling which would be incurred.

However, the results from the laser ablation are very encouraging. The conductive material can be completely removed easily and very rapidly, and the process leaves a very clean line with no sign of the collateral damage seen in the sand ablated lines. An example of a laser ablated line, along with a comparison sandblasted line, is shown in Figure 2.5. It is clear that the laser ablation is much less prone to particle generation than the sandblasted line. This is because the majority of the material removed is vaporized. It is anticipated that changing the process to laser ablation will further reduce the number of particles in the process.

\subsubsection{Bus-bar Application}

The bus bars for the EC device substrates are applied using a modified screen-printing process. The equipment for performing this operation is shown in Figure 2.6. The plate holding the screen can be seen at an angle of about $25^{\circ}$ and this is in the position to enable the glass to be loaded. The screen has already been charged with the conductive material. The glass substrates are placed (one at a time) onto the table beneath the screen,

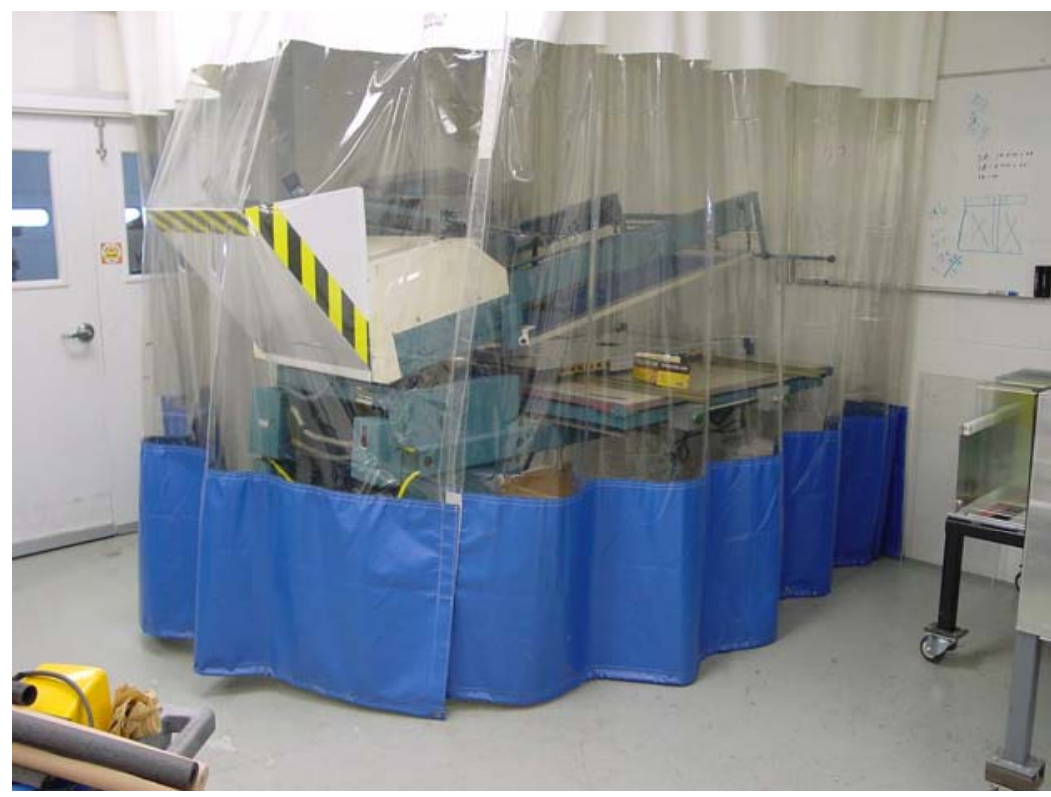

Figure 2.6 - Picture of screen printer showing the plastic shroud, and the proximity to the door discussed in the text. and secured in place using mechanical stops. The screen is then brought down until it is in close proximity to the glass substrate, and the conductive material squeezed through suitably defined openings in the screen with a squeegee and hence transferred onto the glass. The screen is then lifted and the glass unloaded and placed into a storage box. The process is repeated for the next sample.

There are many

possible sources of contamination, one of which is dust blown into the room from the outside, via a door near to the screen-printer. The plastic shroud, which can be seen in Figure 2.6 has been added in an attempt to maintain a slightly cleaner environment, as currently we are unable to house this equipment in a cleanroom. In a further effort to 
reduce particulate contamination, new process procedures have been set-up to improve the repeatability of the process, as well as reduce contamination. These procedures include operation, routine preventative maintenance and cleaning. In addition, the screen-printer has been modified to restrict the mechanical movement, and so reduce vibration and hence contamination.

\subsubsection{Transportation}

Transportation to the tempering site is done via specially designed boxes in an effort to keep the surface of the glass clean. These have been designed so that multiple samples are contained within an enclosed box, and are restrained to prevent them from moving during transportation.

\subsubsection{Washing}

Much of the discussion concerning the modifications we have made to the washer have been made in Section 2.1.1. However, it is worth pointing out that this has had a significant effect on the final number of particles on substrates prior to processing.

Effective removal of particles from the substrate is vital in prevention of pinholes in the subsequently deposited thin films. Microscopically inspecting these types of defects and comparing the size and number of particles on substrates before processing with the number of defects on finished devices established a standard requirement for substrate cleanliness in terms of particulate contamination. Microscopic examination of substrates cleaned by different techniques provided an understanding of not only the best means of removing particles, but also lead to the realization that others were being introduced from some of the washing operations. As a result of some lengthy and painstaking detective work, significant steps have been taken to improve the performance of the typical flat glass washer to the point where it now meets the standard for particulate contamination (for details see Section 2.1.1.1). These steps included: removal of all components within the wetted areas (wash and rinse) which could corrode; replacement of brushes and rollers; addition of a pre-wash section; careful treatment and filtering of the water; regular maintenance; extensive modification to the drive system of the washer; and removal, redesign and reinstallation of parts of the drying system.

\subsubsection{Summary of Particle Reduction Efforts}

This section has described the efforts at particle reduction. The discussion has been very qualitative, as there is no a reliable method of measuring contamination at each of the individual steps. It appears that each of the measures put in place is helping incrementally in the reduction of contamination, and hence the improvement in the quality of the EC devices being produced.

This is supported by the data shown in Figure 2.7 where the average density of white dots measured on a finished EC device is plotted as a function of time. The trend is clearly downwards, indicating the success in contamination reduction. 


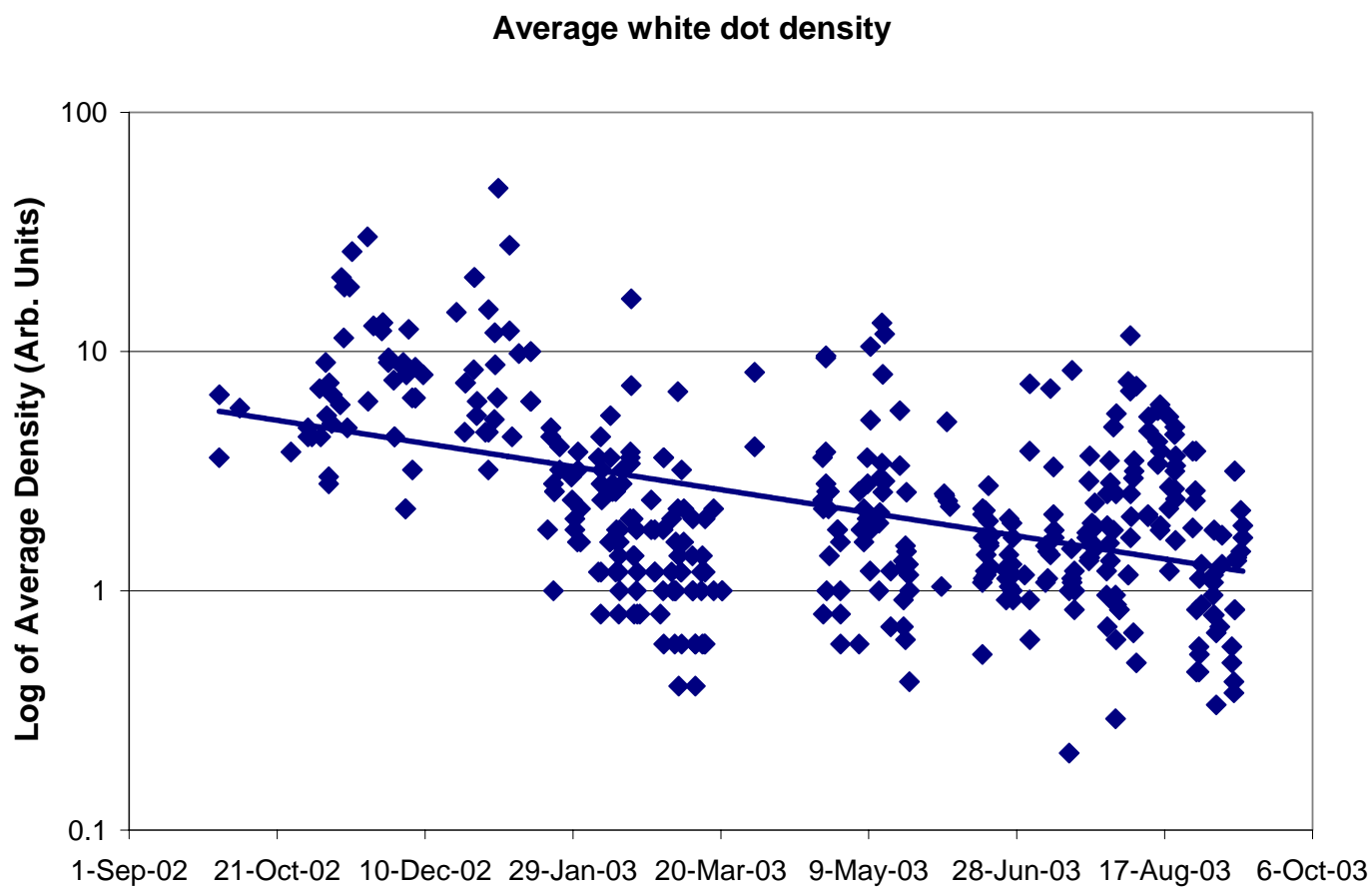

Figure 2.7 - Plot of average white dot density over the last year, showing a significant reduction primarily caused by the contamination reduction measures that have been implemented.

Although some success has been seen in this area, it is by no means satisfactory. Improvements in quality consist of a cycle aimed at ever-higher standards, and this discipline has now been made part of the company philosophy of continual improvement, so the particle reduction efforts, along with all the other quality programs, will continue.

\subsubsection{Manifestation of Point Defects in EC devices}

In this section we discuss the manifestation of the defects in EC devices. Two major defect categories will be addressed: shorts and non-coloring areas.

As discussed in Section 2.1, a short is a defect in which the two transparent conductors are connected together through a low resistance path. This serves to 'short' out the electrochromic effect at the point of the defect, and also in a substantial surrounding region, which can be as much as $10 \mathrm{~mm}$ in diameter. This type of defect shows a gradual change in the depth of coloration from the center of the short to the surrounding (fully colored) region.

Non-coloring areas or white dots are regions where there is little or no coloring during switching, and so there is a very large contrast between the white dot and the surrounding colored region. The size and number of the non-coloring areas can vary and this depends 
on a number of different factors, but it is generally true that the size of the white dot is significantly larger than the particle or defect causing it. Typically, non-coloring areas are less than $1 \mathrm{~mm}$ in size and show an abrupt boundary between the colored and noncoloring areas.

\subsubsection{Shorts}

Work has concentrated on trying to understand the origin of the shorts. The overall strategy was to look at each occurrence of a short microscopically, and then classify this according to its appearance. Each class of shorts was subsequently subjected to chemical analysis to determine the contaminating elements. These could then be traced back to some aspect of the process and hopefully eliminated.

Through painstaking microscopic observation and cataloguing of many hundreds of shorted occurrences, it has been determined that there appear to be several general classes of shorts:

- $\quad$ Stray bus bar material from the screen-printing process;

- Microscopic film delamination precipitated by some sort of contamination;

- Tin speck from the glass production process;

- Glass particles on the substrate;

- Line shorts.

Each of these is now discussed in some detail, covering the analysis done to identify the origin and the remedial efforts made to eliminate or reduce the occurrence.

\section{Stray Bus-Bar Material}

The details of some experimental observations of the shorts produced by this type of contamination are given in Appendix 5.

As a result, very close attention has been given to all areas which could lead to bus-bar material contamination of the substrate, i.e. thick film printing, glass transport, storage, tempering, etc.

\section{Film Delamination or Hole Shorts}

SEM/EDS measurements have been extensively used to understand the morphology and origin for the second class of shorts. Several different elements have been implicated, but the second class of shorts appears to be driven more by the processing conditions rather than the actual level or type of contamination. Appendix 6 shows an example of the analysis results for this type of defect.

Although no definitive picture has emerged, a great deal of knowledge has been gained from this study, and progress towards understanding the shorting problem continues. 


\section{Tin Speck}

An example of a tin speck from the glass manufacturing process is shown in Figure 2.8. This is produced by condensation of tin onto the ceiling of the float glass furnace, which then drips back down onto the glass ribbon. The characteristics of this kind of defect are that it is an almost perfect sphere sitting in a slight depression in the glass. (This depression is caused because the glass is still soft when the tin speck drops onto it).

In the example shown in Figure 2.8, the tin speck is several times larger than the thickness of the thin film stack, and will therefore give rise to a short.

Generally, these defects are detectable by careful visual inspection of the glass prior to processing, provided they are sufficiently large. We now have an inspection procedure that is fairly effective at rejecting all glass with such defects.

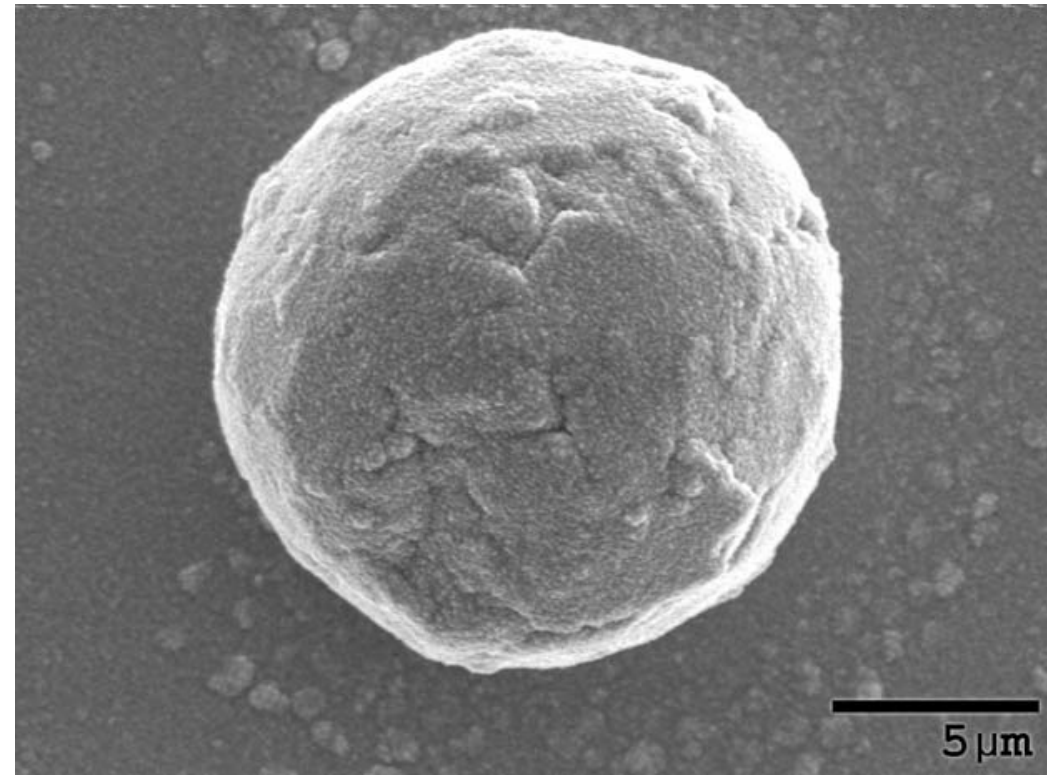

Figure 2.8 - Tin speck on glass surface formed during the glass deposition process. The spheroid is approximately an order of magnitude larger than the electrochromic film thickness and therefore interrupts the thin film stack, creating a localized short.

\section{Glass Fragments}

The fourth category of defect that can lead to a short we label 'glass fragment'. An example of the defect found at the center of these types of shorts is shown in Figure 2.9 below. EDS analysis shows this to have all the constituents of normal float glass.

At first sight, it is difficult to understand why a glass particle would lead to an electrical short, as it is an insulator itself. It is speculated that the films growing near to the glass particle are somehow shadowed so that they are significantly thinner near to the defect. We suspect this because of many observations of fringing effects around the defects, indicating changes in the film thickness at that point. 


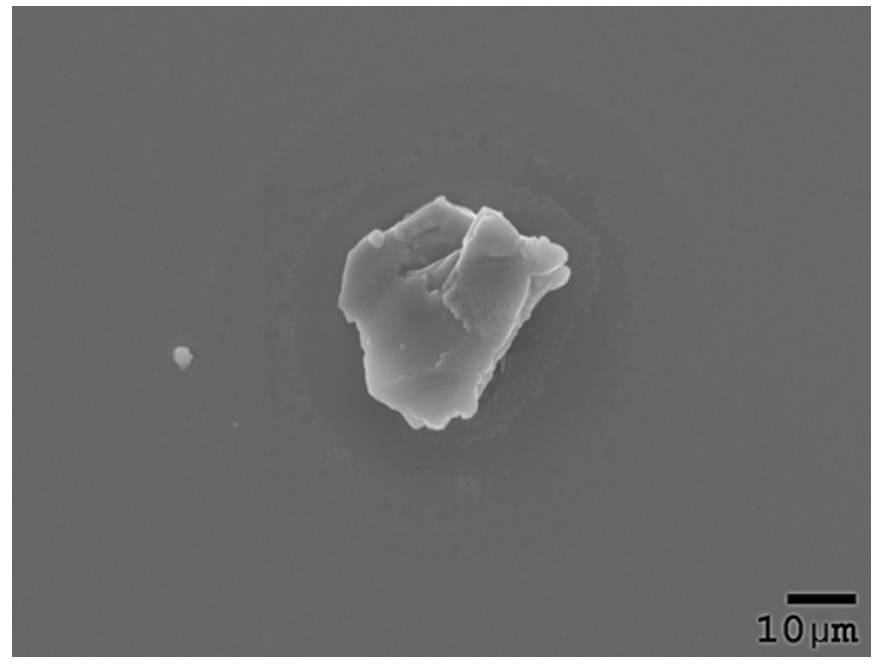

Figure 2.9 - Example of an SEM photomicrograph of a glass fragment found at the center of a short.

The exact origin of the glass particle is not really known. However, it is possible to narrow down the number of origination possibilities. First, there are the glass preparation steps, which we have discussed at some length already. Next, glass breakage within the coater is a fairly frequent occurrence, and so it is likely that glass dust generated during breakages can find its way into the load lock, and hence onto the substrate. We have tried to address these possible contamination mechanisms as described elsewhere in the text.

It may also be the case that glass particles that are weakly bonded to the glass and pop-off during the deposition process could lead to the type of short we have labeled as 'hole' shorts. However, these are generally round in shape, suggesting a different type of particle. The investigation into both of these defect types is continuing.

\section{Line Shorts}

'Line shorts' have a distinctive shape, and an example is shown in Figure 2.10.

The line itself is composed of a metal, which appeared to be in the liquid state when it was 'splashed' onto the substrate. Two different materials have been detected individually in different line shorts.

The occurrence of these shorts tends to be clustered in time: the Pilot Line can operate for a significant period without the occurrence of any line shorts, and then suddenly they will appear in high concentrations. This is thought to be related to the formation mechanism. For example, a number of metallic line shorts were observed for a period between late 2002 and early 2003. The metallic contaminant is used as a standard bonding material for sputter targets, and is used on several of the planar targets. By carefully analyzing the positions of the shorts with respect to the location within the coater, we were able to deduce that the shorts were probably originating from one of the targets. 


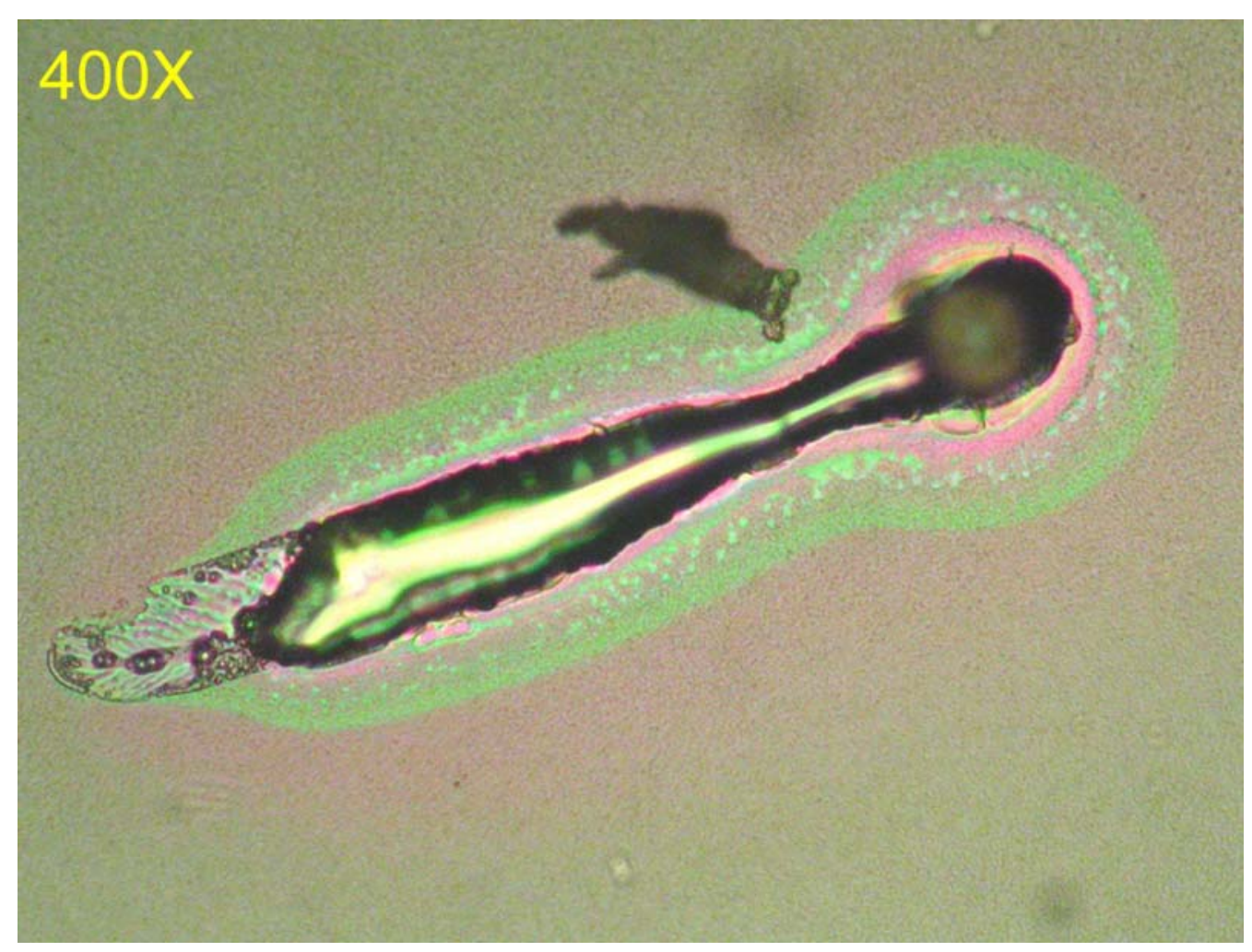

Figure 2.10 - Picture taken through a microscope of a line short, showing a typical shape for this type of short.

Upon venting the coater, it was discovered that this was indeed the case, and that the manufacturer had assembled the target incorrectly. (Usually, if a target is made up of a series of tiles, the gaps between the tiles are 'sealed' to prevent the bonding material from migrating up the gaps between the tiles and contaminating the sputtered film. In this case, the sealing material had been omitted, and metal had found its way up onto the surface of the target). Once the metal is at or close to the surface of the target, it can easily become molten (as it has a very low melting point), and this molten material can then be ejected and end up on the substrate and cause a short. 
Average hard short density at monolithic test

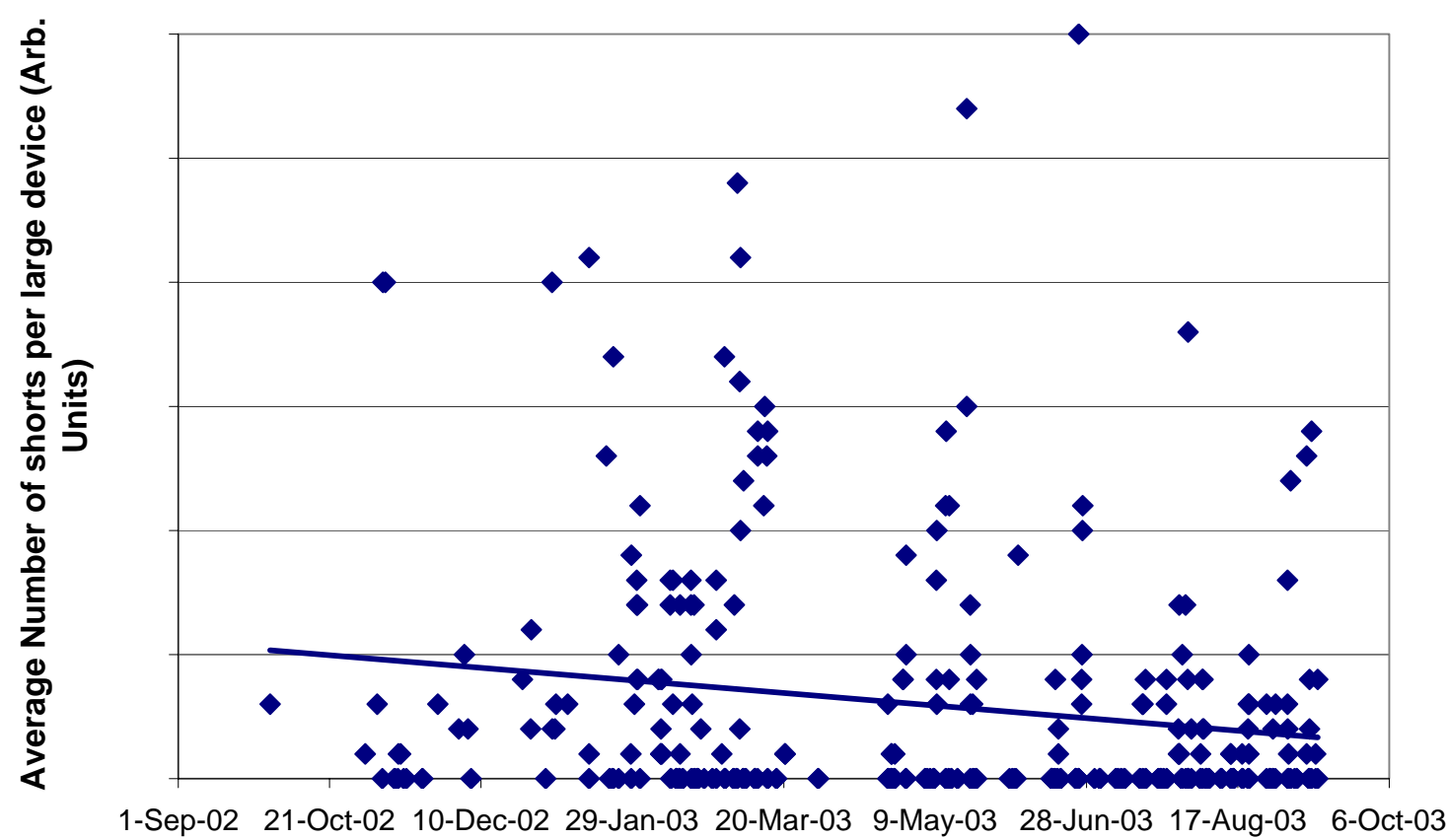

Figure 2.11 - The number of hard shorts measured at initial test for each large device produced in the last year. The line is a best-fit trend line, showing the overall decrease with time.

In this case, the target was immediately replaced, and the occurrence of these shorts disappeared correspondingly.

The origin of the iron is still unknown but present suspicions fall on the edge of the cathode used to deposit the electrochromic material. It is thought this is the case because the analysis of the material in the short shows a proportion of chromium in agreement with that expected from stainless steel. Some pitting in the stainless steel end plate at the end of the cathode used for depositing this layer has also been seen, and although this is not definitive proof that this is the source, there are very few other possibilities for this contamination. Modifications to this area of the cathode in order to address this problem are currently under way. Some further images of line shorts can be seen in Appendix 7.

Overall, it appears as if the general quality efforts are having a small, but significant effect on the level of shorts being experienced. Figure 2.11 shows the number of hard shorts measured at monolithic test as a function of time for about the last year. Although the data is somewhat scattered, there is a significant downward trend. 


\section{Summary}

Although a complete understanding of the highly complicated problem of short generation has not been achieved, some extremely important progress towards it has been made. Clearly, the separation of the shorts into different classes is an important step, and it appears that we are close to eliminating or at least understanding the origin of the shorts caused by silver frit. Further cleanliness improvement measures appear to be having a beneficial impact on the overall number of shorts seen at initial test. We are hopeful that with further careful observation and experimentation, we can begin to understand the mechanism of generation of the other classes of shorts.

\subsubsection{Non-coloring Areas}

As mentioned in Section 2.1, the second particle related defect type seen in EC devices, are referred to as non-coloring areas or white dots. There are two classes of white dot that have been distinguished. The first class we refer to as substrate damage white dots. The second class is generally associated with some sort of particulate contamination, and therefore referred to as particle induced white dots.

\section{Substrate Damage White Dots}

These are circular regions of the EC device ranging from several tens of microns in diameter up to approximately $1 \mathrm{~mm}$ in diameter. These defects are distinguished from shorts because they have a very sharp cut-off between coloring and non-coloring region, compared with a short which has a gradual fall off from colored to non-colored regions, and also shorts are 'leaky' whereas white dots are not. Because the non-colored region is highly transparent - being virtually the same transmission as the bleached state - the contrast offered by the white dot with the rest of the device is huge. This means that these defects are highly visible, and hence must be eliminated for a successful product.

An automatic method for counting the number of white dots on a device has been developed, and therefore values for the white dot density, and the size distribution of the white dots can be determined. This involves taking a digital picture of a representative area of the device, and processing the data contained in the image to arrive at the dot density and the average dot size. This can also be done manually, simply be inspecting the dots under a low power microscope.

The investigation into the root cause of the white dot phenomenon began by looking at the conditions required for the formation of a white dot. Some simple carefully designed experiments $\left(\mathrm{DOE}^{9}\right)$ showed that the following conditions were required for the formation of a dot:

- Presence of the ion conductor layer;

- A heating step prior to deposition of the electrochromic layer.

\footnotetext{
9 'Design and Analysis of Experiments', D.C.Montgomery, Wiley $5^{\text {th }}$ Edition (1997)
} 
These conclusions were reached by performing experiments on partially completed EC stacks in a wet-cell. A wet-cell consists of a liquid lithium containing electrolyte and metallic counter-electrode (CE) in which the sample of interest can be made to be the working electrode (WE). A voltage can be applied between the working and referenceelectrodes, causing a current to flow so that lithium is inserted into the EC layer. Deep coloration of the EC layer can be achieved in this way, and if any white dots are present, they can be seen very easily.

The investigation continued by subjecting some of the defects to microscopic analysis. It was found that the defects were caused by migration of contaminants through small cracks in the transparent conductor layer into the electrochromic films. This presumably occurs under the influence of the heating step, which also helps to explain the almost perfectly circular shape of the white dots, presumably diffusion related. Details of the formation mechanism and some examples of images of the defects are given in Appendix 8. The model for the formation of these defects was then clarified somewhat to the following:

- Presence of a microscopic void or hole in the transparent conductor layer;

- Thermal processing to allow contaminant ion diffusion either onto the surface of the TC, or through the EC stack;

- Presence of the ion conductor layer.

Efforts to reduce the occurrence of white dots could now be directed at (1) improving the quality of the as-received substrate to remove the initial defects responsible for the contaminant ions. This includes better handling and processing to reduce additional damage to the substrate surface ${ }^{10}$; (2) introduction of a barrier layer between the substrate and the EC device to prevent contamination; (3) experimentation with the thermal processing to reduce or eliminate the migration of these contaminants; and (4) modification of the chemistry of the ion conductor to make the process less sensitive to the contamination.

Attempts to make improvements to the as-received substrate have been addressed in some detail in Section 2.1. However, there remains a problem because the defects are too small to be detected prior to EC device fabrication, and it appears as though a certain amount of these defects are inherent to the production process. Other methods for white dot suppression therefore need to be considered.

In order to cover up the defects and prevent contaminant ions migrating into the EC stack, several substrates were prepared with a variety of thin layers of transparent conductors (TCs) sputtered onto the as-received substrate. EC devices were then fabricated on these substrates and the devices inspected for the presence of white dots. The details for this series of experiments are given in Appendix 9. There was a general

\footnotetext{
${ }^{10}$ Our efforts in this direction have been covered fairly extensively in Section 2.1.
} 
increase in leakage currents (and concomitant reduction in the dynamic range) for all the devices fabricated on substrates with an overcoat when compared with control samples fabricated at the same time, and no real impact on the level of white dots was seen. While this first attempt was not successful, US Patent $6352755^{11}$ discloses that other materials such as zinc/tin oxide would be effective barriers. Additional work (NETL IV) is planned to evaluate these materials.

The experiments with thermal processing have encompassed two different areas; the heating during tempering and the pre-heating step for the thin film deposition. For the tempering operation, there is very little latitude for variation in the tempering parameters themselves, as the glass must be heated to a certain temperature and quenched at a certain rate to ensure that it is tempered. The residence time in the furnace has been varied slightly, but this has had no detectable impact on the density or size of the white dots.

Also, experiments have been performed where the preheat temperature for the films has been reduced, but again without any success in reducing the number or size of white dots developed. It has been found that it is necessary to reduce the preheat temperature to a level where the EC device no longer functions properly, if the formation of white dots is to be suppressed. This is obviously an unacceptable solution.

To summarize, the class of white dots that are referred to as substrate induced white dots have been investigated, and the complicated details of the mechanism responsible for their formation has been determined. Improvements in the quality of the substrate preparation, both by working with the substrate manufacturer, as well as improving the in-house procedures, have lead to a significant reduction in the number of this class of white dot seen on finished devices, although there are still an unacceptably large number of remaining defects. In an effort to further reduce the problems seen with this defect, an investigation has started to look at the effects that perturbations to the controlling factors have on the formation mechanism.

\section{Particle Induced White Dots}

As the investigation of substrate damage white dots progressed, it became clear that there was a second class of defects that were not related to substrate damage, but still generated white dots. These white dots appeared to be centered on a particle, rather than a defect in the TC.

Many separate SEM and EDS analyses of particle induced white dots have been carried out. These are summarized in Appendix 10. It is concluded that there are several different sources of contamination that can lead to these types of white dot defects, and the strategy is to identify the cause and eliminate it for each defect in turn. It is assumed that this will lead to a gradual reduction in the level of white dots, and this appears to be the case, as we can see in Figure 2.7.

${ }^{11}$ US Patent 6352755, Finlay et al, March $5^{\text {th }}$, 2002, 'Alkali metal diffusion barrier layer'. 
Inspection of Figure 2.7 shows that it is clear that the efforts being made as a result of overall quality improvements are having an impact on the general level of white dots seen in the devices. There are really no significant points where the improvement can be

Dot Densities on large EC grouped by substrate preparation lot

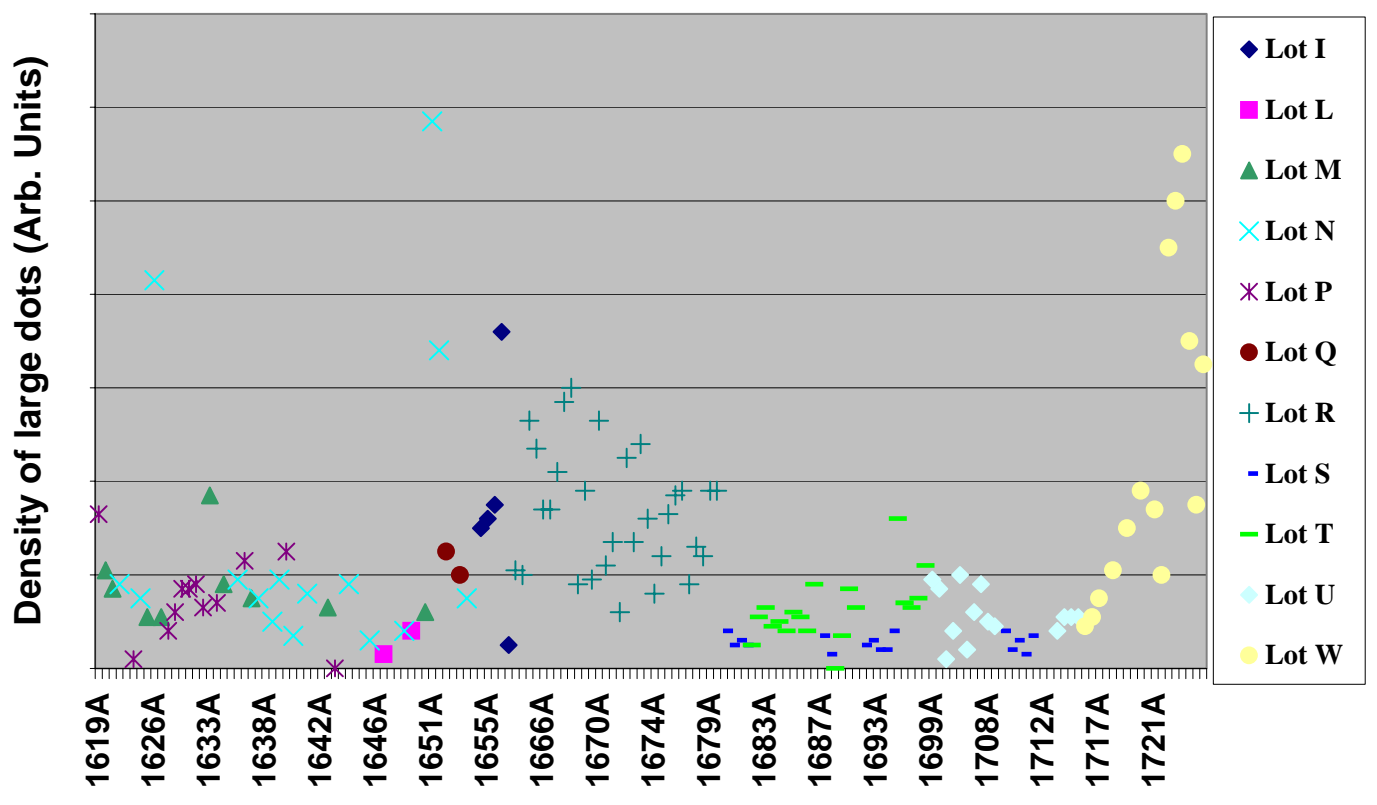

Figure 2.12 - The average white dot density divided into substrate preparation lots, showing the correlation of dot density with preparation lot. It is thought that the variation may be a result of uncontrolled variation seen in the tempering furnace.

linked to a single event, but instead a general gradual improvement can be seen. This is a testament to the quality systems that have been put in place during this project. Further improvement is expected as the evolution of the quality system progresses.

Unfortunately, some of the steps in the substrate preparation are still not under complete control, and this can result in variation. This can be seen in Figure 2.12, where the density of large white dots as a function of substrate preparation batch is plotted. There is clearly a grouping of the data, indicating a dependence on the batch, and it is likely that this is caused by some gross difference in the tempering operation occurring from batchto-batch. Work is being carried out with the operators of the tempering furnace in an attempt to alleviate this problem, but the solution is not obvious, and it would therefore be advantageous to bring the tempering operation in-house. This would require purchase of a tempering furnace, and clearly that is something that will be addressed as part of the scale-up process. 


\subsubsection{Summary of Task 2}

Task 2 has seen a good deal of progress toward understanding the mechanism of the defect manifestation, particularly white dots and shorts. The Task 2 project has been accomplished by a mixture of DOE and analytical methods. This has followed the pattern of running DOE's to identify significant factors, and then investigating differences seen as a result using analytical tools, such as SEM, EDS and SIMS. The main focus has been on reducing two particular types of point defects: shorts and white dots.

As part of this effort, the minimization of particle generation throughout the EC device manufacturing process has been addressed. This has encompassed two significant disciplines: first, the identification and root cause of the defect itself, and second, the elimination (or minimization) of the source of the problem.

Development of methods aimed at reducing the number of particles generated during the process has also started. This has involved optimizing the cutting, seaming and line isolation processes, improving the performance of the washer, redesigning the sputter zone shields, instigating a rigorous schedule of cleaning for the coater, and minimizing the propensity of the target to generate arcs during deposition.

The increased understanding of the mechanisms responsible for both defect types has allowed progress to be made in reducing their occurrence, by addressing the root causes. For example, analysis of a particular type of short, which occurred sporadically, showed that one of the cathodes was responsible. Armed with this information, we were able to work with the target manufacturer to redesign the target manufacturing procedure, and thus totally eliminate that type of short.

The introduction of a quality program during the course of the project has been a major benefit in this area. By combining the discipline imposed by the quality program with a design of experiments (DOE) methodology, the origin of several of the defects that have been problematical for several years have been determined. As a result, the overall level of both shorts and white dots seen on devices has been reduced.

\subsection{Task 3 - In-situ Optical Monitoring}

Changes in the process conditions over the period of a product run account for variations in the optical properties and uniformity of the resultant products. This is true for all optical products made by vacuum processes, but is especially true for electrochromic devices. Large variations or significant process drift can result in the product going out of specification and can require corrective actions on the part of a skilled operator.

Generally, for quality control purposes, the product properties are measured off-line or at the end of the line. This means that there is a significant time lag between the process going out of specification, and the problem being noticed in the output. The result of this delay in recognizing the problem is lost throughput and/or the production of significant amounts of product of marginal quality. There is a need therefore to develop monitoring methods located near to the deposition zones, which give key information about the 
process, the variation in that process, and the product properties at that point. In addition to the improvements in throughput and quality that this would yield, increased knowledge of the process will reduce training requirements for the operators.

The optical monitoring system and its use for in-situ transmission measurements is discussed first. Next plasma emission monitoring, the second topic in this section, is discussed.

\subsubsection{In-situ six-point monitoring system}

In order to reduce start-up times and maintain the product within the required process tolerance, as described in the introduction, a reliable system for in-situ optical monitoring is necessary. This would also have a major impact on reducing the delay between making the process change and measuring the impact on the finished product. This objective can be neatly separated into two areas: transmission monitoring, and plasma emission monitoring. In the first area, monitoring the transmission of the films as they are deposited allows instant feedback to the operator indicating whether changes to the process are required. Second, Plasma emission monitoring of the actual process allows the operator to ensure that the process conditions are constant, both in terms of sputtering rate and thin film composition. First the construction and installation of the monitoring system itself is discussed, and then a few applications are discussed. Finally, the most recent upgrade of the monitoring system is considered.

\subsubsection{Design and installation}

It was important to be able to examine distributed spectral information on a real-time basis, so a simple six-channel spectrometer system using optical fibers was obtained. This was capable of monitoring several different points in the process simultaneously, or six points across the width of the coater in order to measure the uniformity of any particular layer at any time. This system is highly flexible, and is reconfigured simply by changing which fibers are connected to the spectrometer inputs.

The spectrometer system allows six spectra to be acquired simultaneously. A schematic layout of the system is shown in Figure 2.13. This is done by using six separate spectrometers (separate dispersive elements, with one light source), and multiplexing the output signals, which are read directly into a PC using a high performance A/D card. The spectrometer allows spectral measurements to be taken between 200 and $1100 \mathrm{~nm}$, with a resolution of $0.3 \mathrm{~nm}$. Spectra can be acquired in as little as $3 \mathrm{~ms}$ or integration can take place over times up to 60 seconds, depending on the amount of light available. Fiber optic cables were specified and purchased allowing optical signals to be taken into and out of the vacuum system, and specially designed vacuum flanges were fabricated to permit this. Collimating lenses are used for light collection in all of the applications to boost the signal to usable levels. 


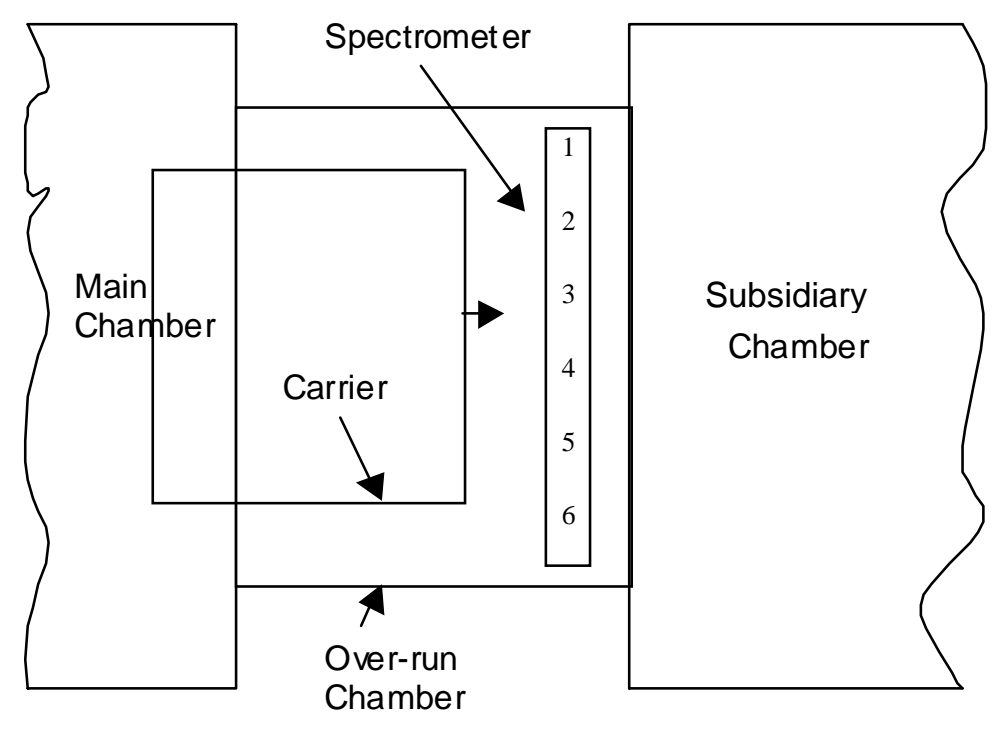

Figure 2.13 - Schematic diagram of the layout of the spectrometer system. The individual channels are labeled 1-6, and the carrier is shown schematically moving under the spectrometer.
The efforts at in-situ film monitoring have been restricted to transmission measurements. This is due to the difficulty in obtaining a reflectance measurement that can be repeatably and reliably calibrated to $100 \%$. However, significant progress has been made using transmission measurements, such that the requirement for reflectance measurements has been practically removed.

Several designs of the fiber-optic based in-situ monitor have been made, and have arrived at two different designs, which are equivalent in terms of the measurement.

Each has several common features, such as the spectrometer. The major difference is that in the first design, light is fed through into the vacuum system by means of a fiber and a vacuum feed-through, whereas the second design has a light source mounted inside the vacuum chamber itself. The main advantage of the first design is that there is little or no maintenance required on the part mounted inside the chamber, provided it is shielded correctly to prevent coating build-up on the optics. It can therefore be used in parts of the vacuum system where venting is undesirable and infrequent, and where replacement of a burnt-out bulb would be a major inconvenience. The disadvantage of this arrangement is that it requires more fibers and feed-throughs, which add to the cost. The second arrangement is used primarily in the overrun chamber (see Figure 2.13), which can be vented independently of the rest of the vacuum system should the need arise.

The optical monitor consists of a rectangular frame made of aluminum, through which the carrier can pass unimpeded. In the second version, six lights are mounted in holes drilled in the lower section, spaced equidistantly across the coater, and are powered by a low voltage source fed in from outside the coater via an electrical feed-through. The lamps are shielded so they only illuminate a detector mounted directly above, so there is no possibility of crosstalk. The detector consists of a focusing lens that couples the light into a fiber. Each of the fibers is brought to a specially designed, custom built, optical feed-through. The light from each fiber is then fed into a dedicated spectrometer, and the results displayed as transmission on a computer. 
Maintenance is carried out on a regular basis, and the lamps are replaced on each occasion. The optical monitor has been designed so that there is no direct line-of-sight from the coating source to any of the optics, and so the requirement for continually cleaning the optics is removed.

It is possible to obtain good stability of the lights in vacuum, however they require approximately 30 minutes to warm up and reach equilibrium. Immediately before the lamps are turned on, a 'zero' baseline reading is taken, and once a constant illumination has been reached, a $100 \%$ reading is taken. This is sufficient to calibrate the measurement, and accommodates any slight changes in light output that may occur on a day-to-day basis.

The output from the spectrometer is processed and the results automatically stored, and as will be seen later, this data is available via a network for post-deposition analysis. The spectrometer is conveniently located between the CE cathode and the dopant source.

The following two sections describe work done using this monitoring system. Section 2.3.1.2 describes the work on the CE process, followed by Section 2.3.1.3 and Appendix 12 describing the work on the doping process.

\subsubsection{CE process}

This is a fairly straightforward, but nonetheless important, application of the optical monitoring technology. First, the transmission of the sample prior to CE deposition is measured. Then the transmission of the sample at various stages of CE deposition (i.e. after each pass across the target) is also measured, and the required line-speed (or target power) for the final pass calculated. The calculation is very simple, and has been shown to give very good correlation between measurement and prediction.

Some typical relative transmission data for the CE deposition process are shown in Figure 2.14. In this measurement, the samples pass over the detector at a constant rate. It is seen that the transmission starts at around fairly high, and is reduced in the subsequent measurements. Also, some lead to trail non-uniformity is detected, which has subsequently been attributed to pressure variations during the deposition. Introducing a pressure controlled oxygen flow-controller for this process has substantially reduced this problem.

Transfer of the monitoring technique into manufacturing should be very straightforward. Each pass across the current target can be considered equivalent to a pass across a separate cathode in the manufacturing plant. An optical monitor will be required between each successive cathode, and the same simple calculation can be used to rapidly tune the process. In summary a method has been developed for in-situ monitoring of critical layers in the process. This is fully transferable to the anticipated next stage manufacturing line. 


\subsection{Oxidation state dependence}

The absorption of the CE film is an important indication of the state of oxidation of this layer - larger absorption indicates a more oxidized film. It is important to maintain consistency of the oxidation state of this film to assure uniform performance of the final product. We have carried out several experiments to determine the effects of gaseous additives to the plasma on the oxidation state of the CE. The results are described in some detail in Appendix 11.

Run 1346 CE Only

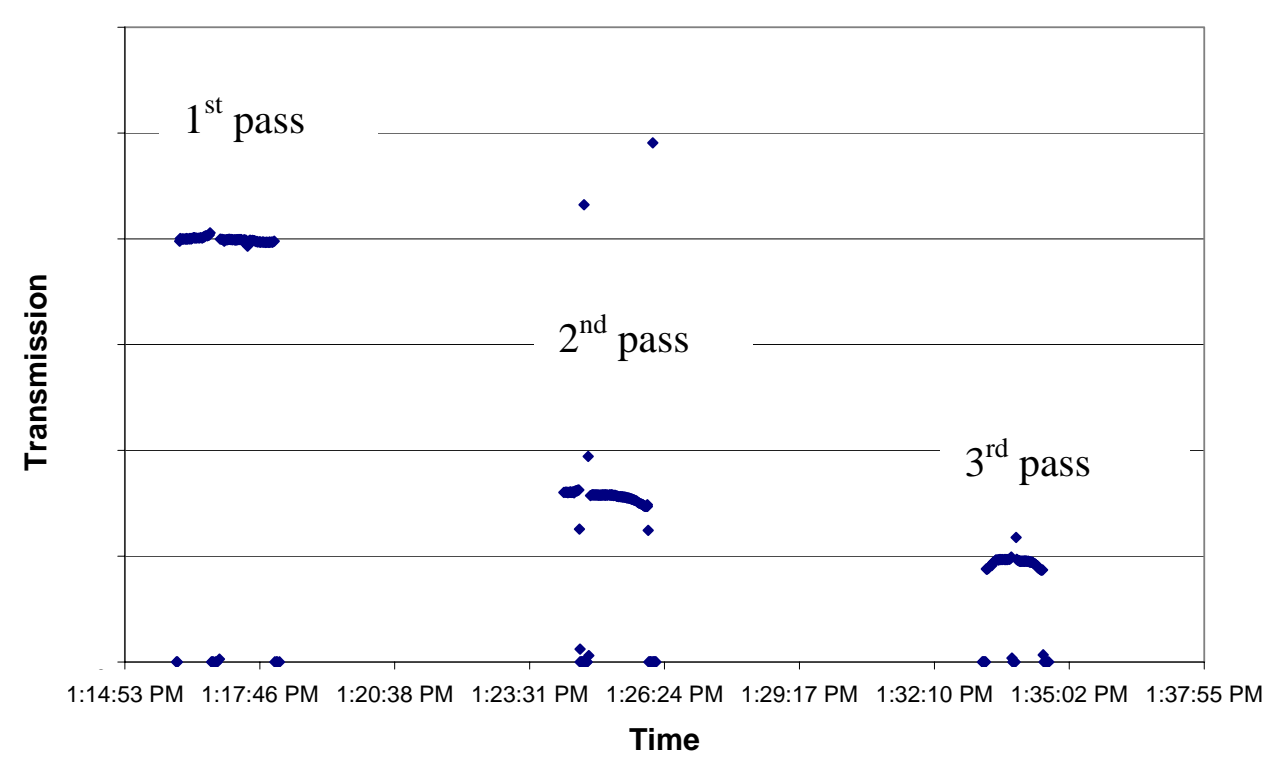

Figure 2.14 - Example of the measurement of the in-situ transmission of the CE layer during deposition.

The optical properties of the $\mathrm{CE}$ were found to be sensitive to nitrogen and $\mathrm{CO}_{2}$ and insensitive to water. It is therefore important to ensure that the background contamination is held below a specified level, so that the effect of these contaminants on the process remains at a constant level. A procedure has been initiated where the base pressure of the system is monitored and the process can only be run if this reaches a predefined level.

\subsection{Initial temperature dependence}

We also found that the absorption of the CE film depended on the initial substrate temperature. This is illustrated in Figure 2.15, where we have plotted the final transmission (measured using the in-situ optical monitor) against the initial temperature of the substrate. The substrate temperature is measured while the sample is in the load lock using an optical pyrometer. 
The reason for the variation in temperature is twofold: first, the temperature of the loadlock will depend on whether the heater has been used recently and is therefore still hot. Second, immediately prior to CE deposition, the glass is removed from the oven having had the previous film heated. Obviously, there is room for variation in the substrate temperature from both of these sources.

Transmission after CE as a function of initial substrate temperature

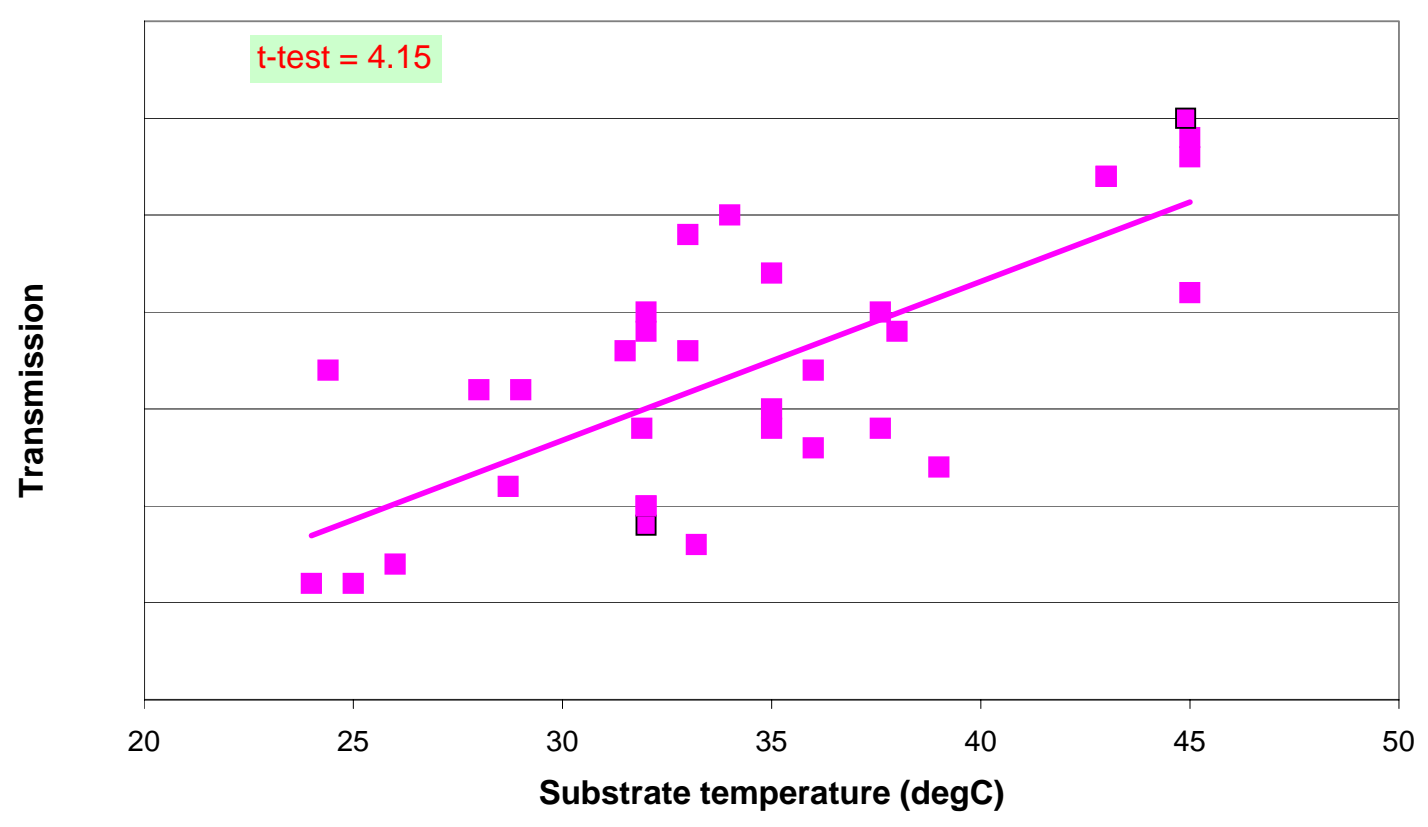

Figure 2.15 - Relative dependence of the final transmission of the CE upon the initial substrate temperature.

It can be seen from Figure 2.15 that there is a clear dependence of the transmission on the temperature. In this case, a significant value ${ }^{12}$ for the $t$-test is calculated. It is also clear that there is a good deal of scatter of the points around the line, suggesting that the temperature is not the only uncontrolled variable operating here. There may be some uncertainty in the temperature measurement, as the pyrometer is known to be somewhat inaccurate at the low end of the temperature range. Further work is continuing to understand the temperature effects.

\footnotetext{
${ }^{12}$ Values of greater than 3.0 for the $t$-test are generally used to denote a significant dependence of the two variables. In this case, we see a calculated value of $t=4.15$.
} 


\subsection{Correlation of ex-situ measurements with in-situ data}

In the current process, the layers are deposited without venting the system. This means that although it is possible to collect in-situ transmission data characteristic of the whole stack, it is not possible to determine the detailed optical indices of the individual layers. Although this is not necessarily a problem for process control, it is an issue for process development, where it is important to understand the effects of differences in individual layer properties on the finished device performance.

\section{Transmission of CE witness pieces}

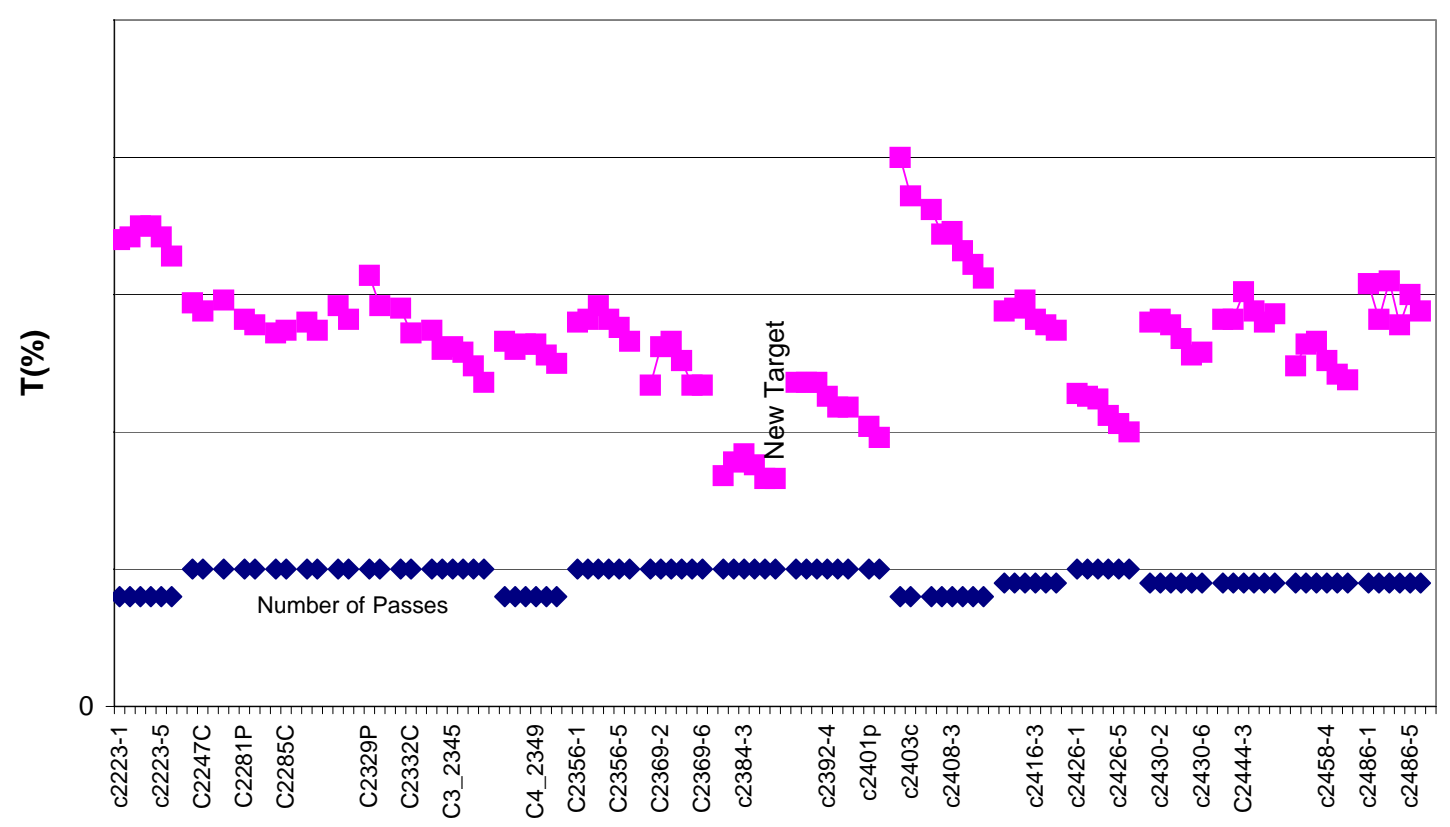

Figure 2.16 - The relative transmission of CE witness pieces labeled by run number for a period of approximately five months.

Dedicated CE depositions are being done to obtain single films on plain glass substrates. These films are then characterized by transmission and reflection measurements, and the values for thickness and optical constants extracted. Figure 2.16 shows the measured transmission of such films plotted as a function of time. Each string of connected points represents one separate deposition run, comprising six samples ranged across the width of the coater. These runs are approximately one week apart. Some experimentation with the number of passes (four to five) across the target has been done. This can be expected to give a variation in thickness, and hence transmission, and so this information is also shown here. 
A significant variation in transmission is obtained immediately after installation of a new CE target, and this is attributed either to a 'burning-in' of the target surface, or some sort of outgassing of the porous target material itself. These targets have a density of around

Thickness of CE witness pieces

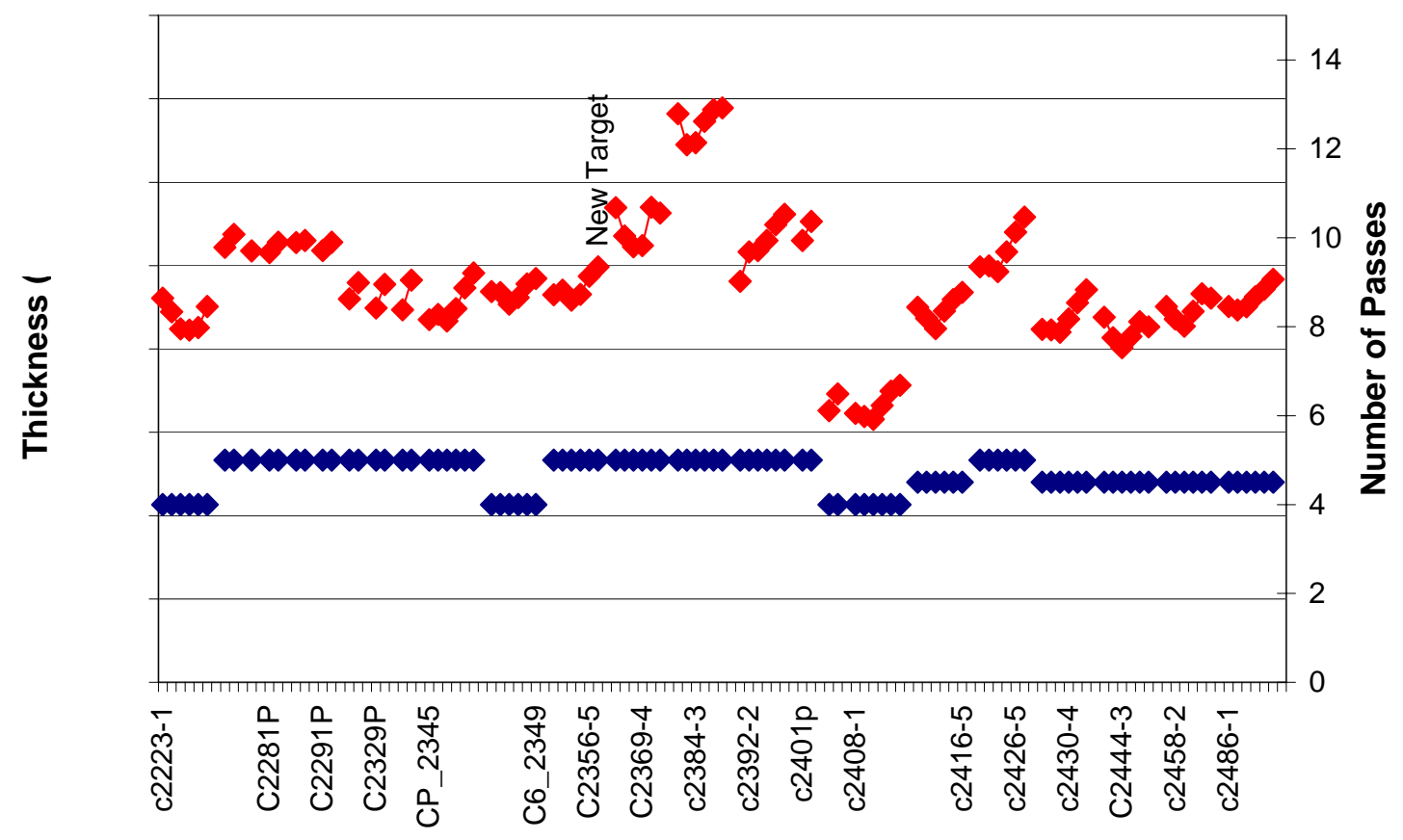

Figure 2.17 - The relative thickness of the samples measured by the method outlined in the text, for samples whose transmission is given in Figure 2.16.

$75 \%$. However, after a settling period, very acceptable repeatability for transmission is obtained. This, however, does not tell the whole story. Figure 2.17 shows the variation in thickness for the same films, and Figure 2.18 shows the calculated $k$-value at $500 \mathrm{~nm}$ (this is taken to be a representation of the absorptivity of the film). 
A clear run-to-run variation in the thickness and absorptivity for these films is seen, indicating a process variation which is to some extent (as far as the overall transmission is concerned) self compensating. Without this off-line measurement, this variation would have remained hidden.

In addition, a variation of each of the parameters within a given run is seen, indicating a variation related to the target and cathode assembly. The thickness variation, shown by sequential measurements that decrease and then increase across the target, has been partially correlated to the variation of the magnetic field strength of the cathode itself. Steps were taken to improve the uniformity of the magnetic field, which resulted in an increase in the uniformity of the coating within each run as anticipated. However, the transmission on such things as substrate temperature (Section 2.3.1.2.2) and background

$\mathbf{k}(500 \mathrm{~nm})$ of CE witness pieces

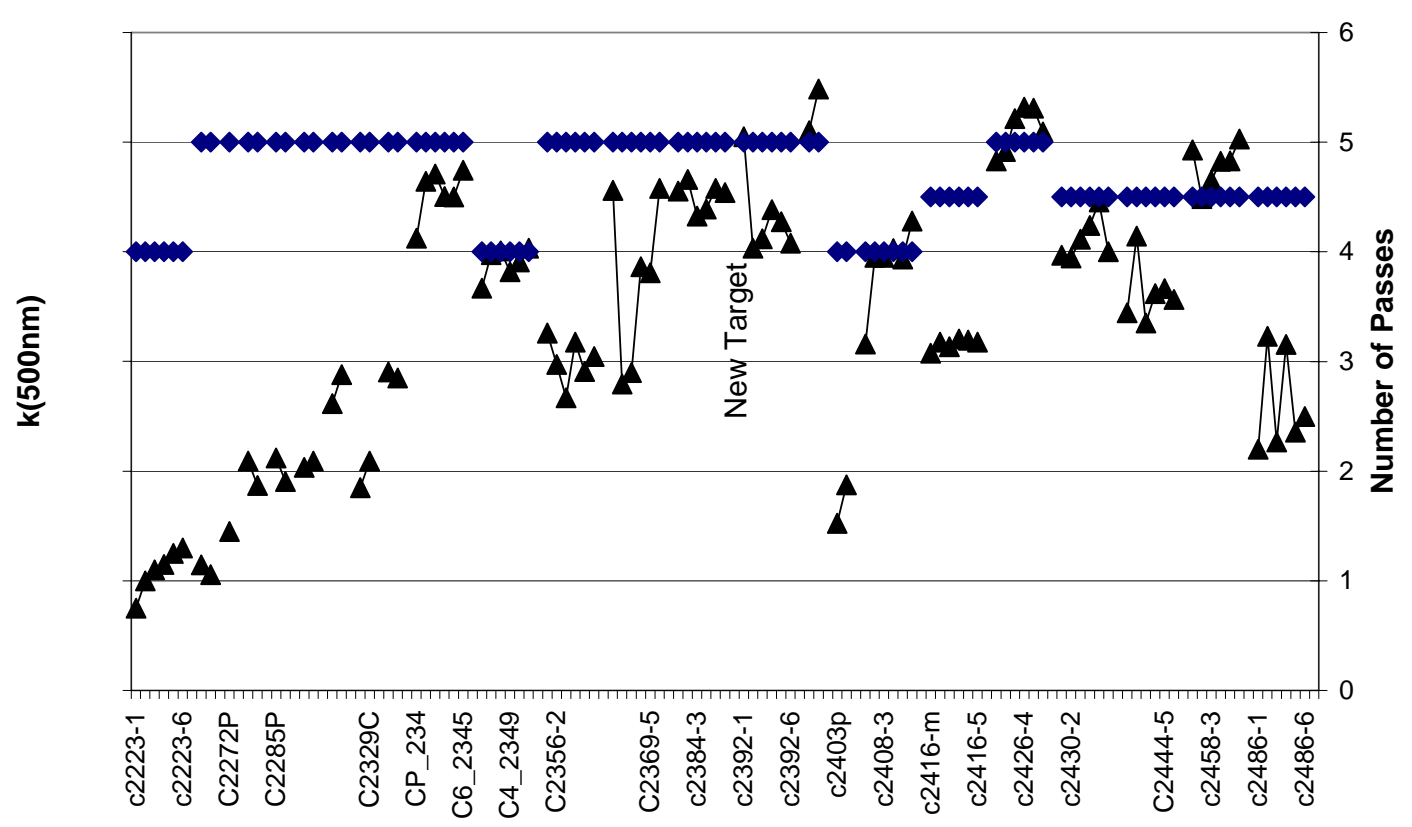

Figure 2.18 - The value of $k(500 \mathrm{~nm})$ of the samples measured by the method outlined in the text, for those samples whose transmission is given in Figure 2.16.

gas contamination (Appendix 11). As a result of these studies, steps have been taken to reduce the variation seen in the optical properties and thickness of the films, and the rewards have been significant increases in repeatability.

An example of improvements in uniformity has been seen from measurements of the transmission of the witness pieces, which only measure uniformity across the coater. The non-uniformity has fallen from an average of $9 \%$ in June of 2002, to an average of $6 \%$ in September of 2003. In addition, the lead to trail uniformity has been improved, principally by use of pressure control for the process. 


\subsubsection{Lithium Process}

Over the course of this project, a large focus has been placed on control of the lithiation process. This is because the level of dopant is crucial to the overall behavior of the EC device. The work done in this area is discussed in Appendix 12.

A next-generation optical monitoring system for the coater has been developed. In addition to the existing six fibers for spectroscopic measurement, two 40-point broadband visible light measurement systems have been designed and installed. With these systems, much more detailed data about the uniformity of all our films, including the lithiation process, can be collected.

\subsubsection{Plasma Emission Monitoring}

Plasma emission monitoring (PEM) is used in the optical coatings industry as a diagnostic and a process-monitoring tool, but is currently not widely adopted due to significant practical integration difficulties and some process issues. However, the information that is potentially available using this technique offers a great deal of promise for process monitoring and control.

Initially, a literature survey was carried out to determine if there was a commercially available PEM, which would suit our purposes. It was found that the few that were available were either prohibitively expensive, or not suitable for the requirements of this project or both. It was therefore decided, after several detailed technical discussions with our partners, that we would specify our own custom system. The system was successfully specified, purchased and installed onto the Pilot Line sputter coater, where it was initially used to monitor the EC deposition: a good example of a reactive process.

Initially, the emission lines from the EC process were much more poorly resolved. This required a slight redesign of the collection optics. In addition, it was found that the intensity of the overall signal varied in an oscillatory manner, due to the target rotating slightly off-axis. It was also found that the intensity of the signal was a function of the position of the carrier, and can be attributed to a change in the pumping pathway as the carrier moves in and out of the coating zone. This resulted in changes in pressure and hence PEM signal intensity. Careful averaging of the signal when the carrier was within the deposition zone over several target rotation cycles gave a useable signal.

A typical plasma emission spectrum for EC emission in a plasma composed of oxygen and argon is shown in Figure 2.19.

Some evidence that the integrated signal can be correlated with the deposited film thickness has been seen, but this needs to be confirmed over a wider range of deposition conditions. 


\section{plasma sustained in Argon with a partial pressure of Oxygen}

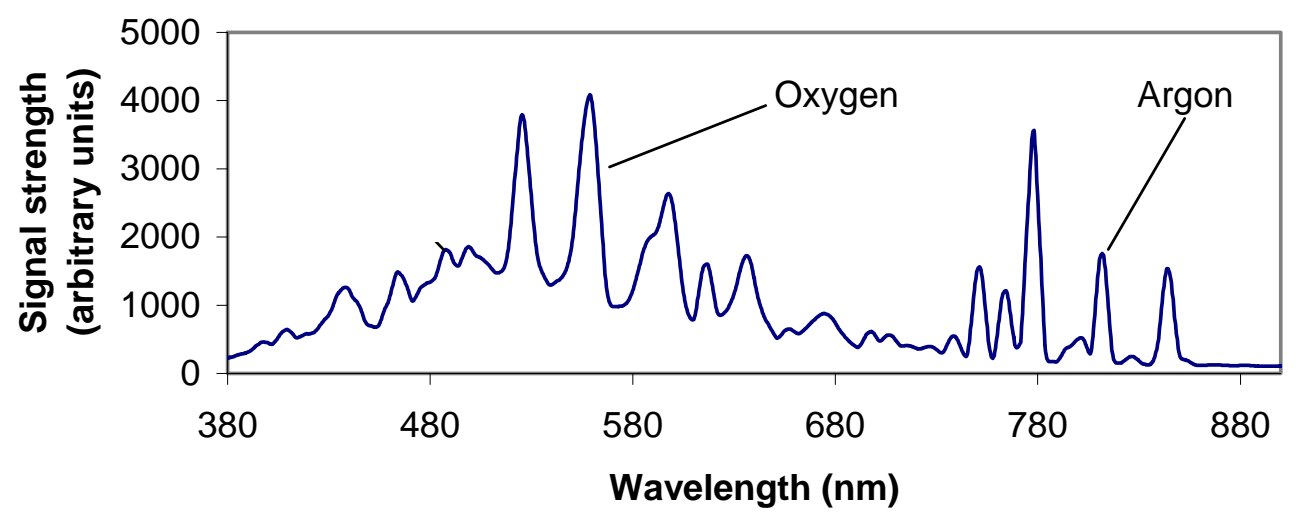

Figure 2.19 - Boosted plasma emission signal from EC plasma, showing improved resolution over the unboosted signal.

As both the uniformity of deposition and rate, are important, steps have been taken to extend the PEM technique to address this. Folding mirrors have been installed to allow an observer looking in from the side of the coater to view the profile of plasma emission along the length of the sputter deposition zone. This means the mirrors are at $45^{\circ}$ to the viewer. Figure 2.20 shows the folding mirrors from the side of the coater, as installed. The upper mirror provides a view of one side of a sputter deposition zone, showing bright plasma emission, where the center of the deposition zone is at the right. Digital cameras aimed at each mirror, and equipped with line filters for atomic emission selectivity, will record the plasma emission profile across the deposition zone, so that a plasma emission profile can be

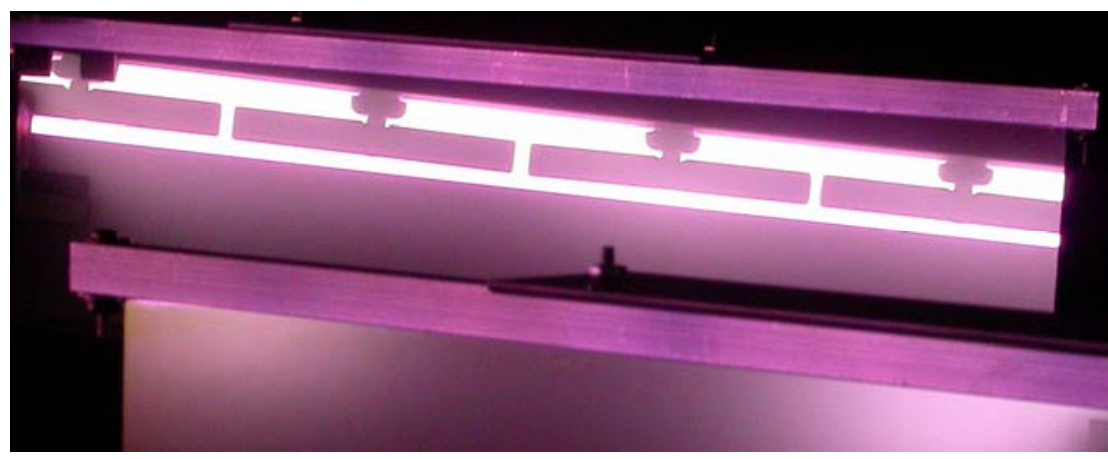
monitored in real time. This will permit real time feedback control, so that deposition uniformity can be controlled adaptively.

Figure 2.20 - A view of folding mirrors installed in the coater to permit real time monitoring of the plasma emission profile. 


\subsubsection{IC thickness Monitoring}

The refractive index of the IC film is near to 1.5. Therefore any method using transmission to determine the thickness will require either a high refractive index substrate, or deposition onto a very well characterized thin film with higher refractive index than the IC layer. Attempts to use transmission were unsuccessful, so a reflection technique has been developed.

In the present method, a film of the IC is deposited onto one half of a silicon wafer and processed normally. The reflection from both the uncoated and coated sections are measured using a single channel version of the spectrometer used to measure in-situ transmission. The thickness of the IC layer can easily be deduced from these measurements. In fact, the requirement has been reduced from fitting the whole of the measured reflectance spectrum to obtain the thickness, to being able to deduce the thickness from a measurement at a single wavelength using a look-up table. This allows rapid and accurate determination of the thickness of the layer.

The accuracy of our measurements has been checked by comparing it with the thickness obtained by ellipsometry, and both have been found to be in good agreement. The thickness monitor is now used on a routine basis for the evaluation of the thickness of all IC layers.

\subsubsection{Summary of Task 3}

Task 3 focused on the development of in-situ tools to optically monitor the deposition of thin films as deposited. Two main areas were investigated: thickness monitoring by transmission measurements, and process stabilization using plasma emission spectroscopy. As a spin-off, a reflection method for determining the thickness of the IC layer has been developed.

Implementation of in-situ transmission measurements has been very successful. These measurements are routinely used to monitor the thickness of the most critical layers as they are deposited. Initially, a six-channel spectrometer system was implemented, and this has been used to monitor uniformity as well. This led to installation of an electronic system for real-time uniformity modifications, and finally to a 40-point optical monitoring system. As a result of the work here, the manufacturing plant will rely heavily on in-line in-situ optical monitors.

Work with the PEM has shown that the need to ensure that the signal obtained is not influenced by process parameters such as pressure or target rotation. We are currently in the process of installing systems that allow monitoring of the plasma intensity over a distributed region for real time feedback and adaptive control. Further development work is required to demonstrate the usefulness of this technique for the next stage of our development. 


\subsection{Task 4 - Integration of Inspection and Monitoring Tools}

The ambitious objective of this task was to integrate the range of on-line inspection and monitoring techniques developed in Tasks 1-3 into a complete system for process and product quality control.

The initial control system concept involved taking inputs simultaneously and in real time from the outputs of the different monitoring tools, processing the data and then displaying meaningful information. This would then allow an operator to monitor process stability and product quality and take corrective actions if necessary. The next level of control system sophistication would involve developing more detailed correlations between final product quality and the array of process parameters. A database would be constructed using real time inputs and information derived from past experience. The goal would be to identify product defects and use the multivariate inputs and the experiential database to suggest corrective actions.

The outputs and results from Tasks 1-3 have been and are still being used as a basis for selecting which tools are appropriate for incorporation into the real time process monitoring and product inspection system. This activity is focused on specification of instrumentation for our first production machine. Criteria that we have used to evaluate the suitability for use include integration limitations, type and quality of information provided, and response time. The Pilot Line is being used as a prototype minimanufacturing plant, and the instrumentation evaluated from that perspective.

At the beginning of the project, the Pilot Line coater had no data gathering facilities. The operation of the coater was controlled by a Programmable Logic Controller (PLC), which was in-turn controlled by a personal computer (PC). There was no optical monitoring, and no centralized database for data storage.

The following describes the work which has been done in instrumentation and data gathering during the project, and examples of the benefits of this work are shown.

\subsubsection{Hardware Installation}

The first task was to develop hardware and software architecture to bring outputs from a variety of instruments and monitors to a central location for further analysis.

Furthermore, it was desirable to provide a real-time feedback of the relevant information to the operator, so that key process parameters as well as product performance and quality could be monitored, and action taken if required.

Figure 2.21 is a schematic representation of the Pilot Line Coater and associated data gathering equipment. There is a local area network (LAN) set-up to provide an easy method of sharing data. Each computer (PC) is connected to the LAN, and the data collected and stored locally. This allows easy access to the data from remote locations, and also provides convenient and reliable data backup facility. 


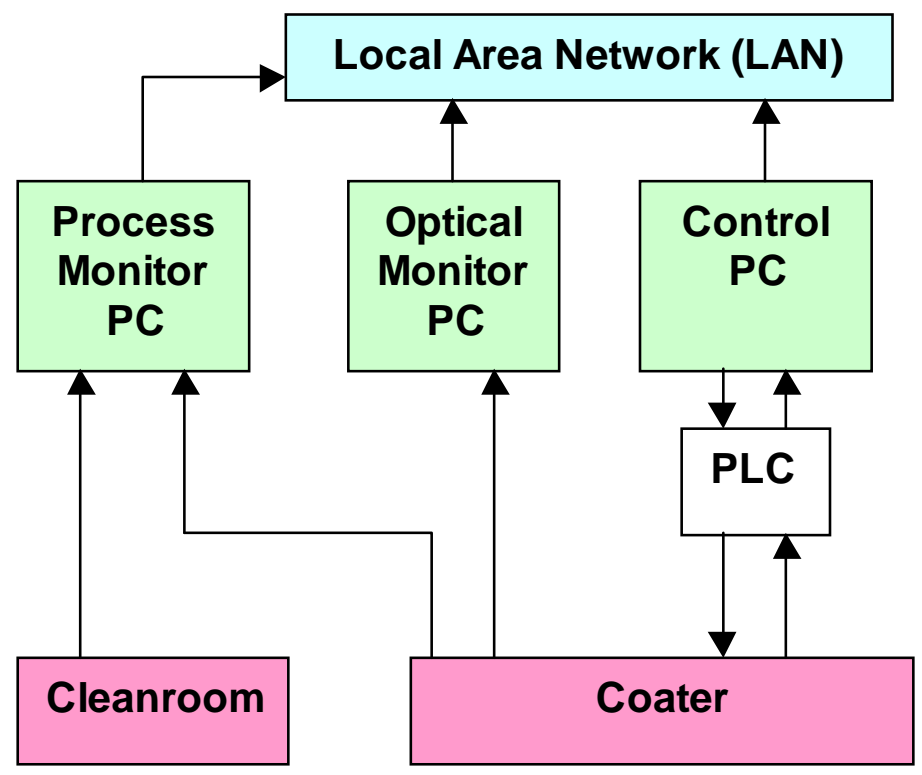

Figure 2.21 - Schematic representation of the data collection setup for the Pilot Line coater and cleanroom. Control signals are sent from the Control PC to the coater via the PLC, and the process monitor and optical monitor PCs record data returned from the coater. These data are then passed to the LAN for analysis.

The coater has been instrumented to allow measurement of many different parameters. These include: pressures, gas-flow, power supply values, valve and pump operation, temperature, drive-speed, etc. Additionally, the cleanroom has been instrumented, so that temperature and humidity data are also collected and stored centrally. These two data collection operations occur through a dedicated process monitoring PC, which is in turn connected to the network. The optical monitoring set-up - discussed in Task 3 - has its own dedicated PC for data collection, which is again connected to the LAN. Finally, yet another PC controls the operations of the coater, this time via a PLC, and some additional signals are passed to the LAN via this PC.

As an example of the level of integration achieved, roughly 20 key process parameters are monitored via the Process Monitor PC. Each parameter is monitored many times every minute, and this allows trends in the data to be seen, or to detection of noise in the signal which otherwise have been impossible to see. Almost all of the data which has been taken for these key process parameters has been useful at one time or another, so further monitoring is being implemented. 


\subsubsection{Process Data integration}

Obviously, with the level of computerization, the flexibility in measurement interval is practically unlimited. Data are stored in Excel spreadsheets, and can then be analyzed with a variety of software tools. We are currently implementing SPC charting for all the critical variables.

The monitoring frequency is important in allowing previously hidden variations to be uncovered that could not otherwise be detected by manually recording data. An example of this was a variation in the cleanroom temperature. Usually, the temperature in the cleanroom was recorded periodically throughout the day. From these measurements, it was impossible to determine whether there was any significant short-term temperature variation. After configuring the data monitor to record the temperature approximately once every minute, it soon became apparent that there was a periodic fluctuation in the cleanroom temperature of $\pm 2.5^{\circ} \mathrm{C}$ with a characteristic periodicity of a few minutes. After detecting this variation, we were able to retune the temperature controller to reduce the observed variation to $\pm 0.2^{\circ} \mathrm{C}$.

\subsubsection{On-line optical monitor integration}

A major part of the data management project involved integration of optical monitoring into the control system. This has been a multi-step process, beginning with a stand-alone PC controlling the six-channel spectrometer. The aim is to use data from the spectrometer to facilitate a closed loop process, whereby control of the deposition occurs automatically, and as a direct result of feedback from the optical monitor.

Significant progress has been made towards this goal. The first requirement was to determine the important parameters for the process, and this is discussed in some detail in Section 2.3 and the relevant Appendices.

The next requirement was to enable the relevant data to be made available to the operators, and to allow them to make decisions regarding the process. This required writing software to (i) control the spectrometer, and (ii) make the data available for processing via the LAN. In practice, determining the next step in the process meant deciding whether additional deposition was required or whether to stop film deposition. These decisions were based on values obtained from the transmission measurements and initially required a good deal of judgment on the part of the operator. As the understanding of the process has grown, and uniformity of film deposition has improved, more automation is possible, thereby reducing the dependency on skilled operators, and removing the possibilities of variations in the process.

Currently, data from the optical monitor is processed automatically so that the options for further processing are presented to the operator as control instructions for linespeed or power. The operator then 'closes the loop' by using the information so generated, to complete the next step of the process. Closing the loop completely requires further software to be written taking the output of the optical monitor and using it to control the 
sputtering machine. However, we currently do not have the hardware ${ }^{13}$ to allow the machine to be run completely automatically. This implementation is being considered for the next stage manufacturing coater.

Integration of the 40-point optical monitor into the overall scheme is currently underway. This will improve deposition uniformity using the multiple electrode system, and will mean that the uniformity can be both monitored and corrected in real time, thus eliminating the need for several costly and time consuming vents.

\subsubsection{Quality Improvements}

Throughout this report, there has been a recurring theme of quality improvement, from substrate preparation, through to device processing, and all the steps in-between. This is

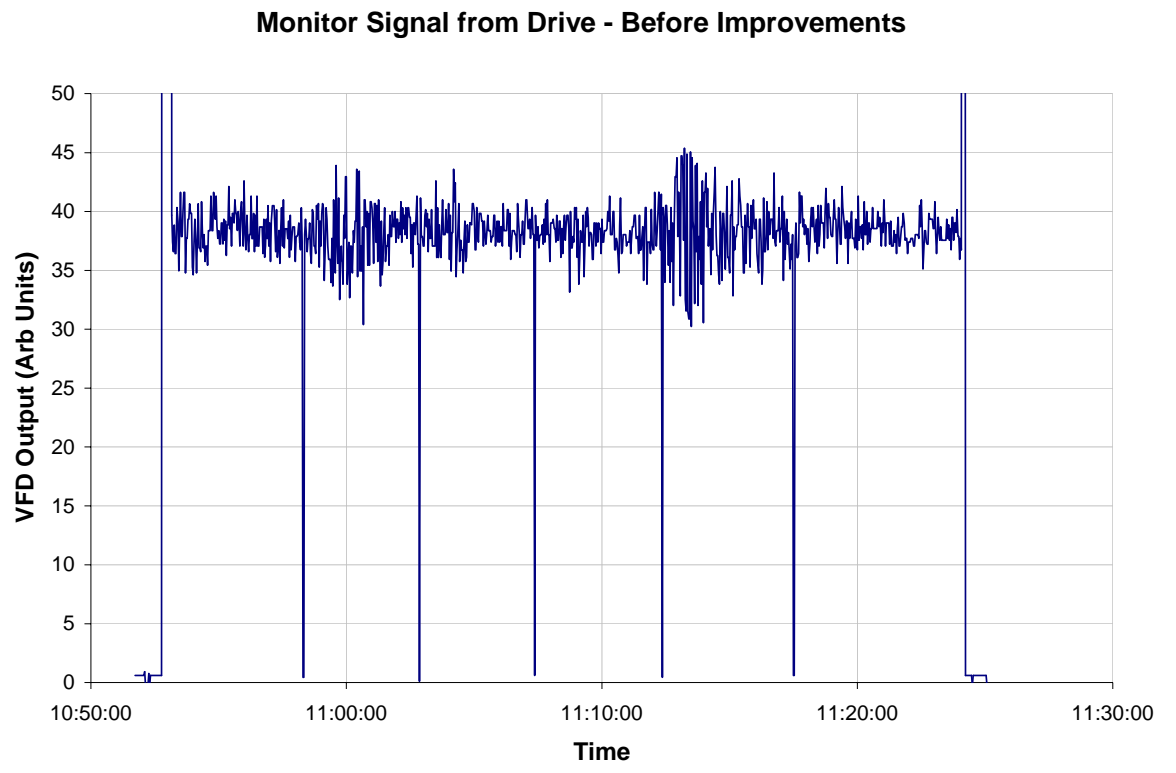

Figure 2.22 - Control signal responsible for drive-speed prior to the changes discussed in the text. really

encapsulated

in the

monitoring

and integration

project, and is

best illustrated

by an

example.

The linespeed

is controlled

by a signal

generated by

an analog

output from

the PLC. This

signal is sent

to the drive

motor

controller, thus

determining

the linespeed.

One of the

signals which we monitor is that control signal from the PLC. Figure 2.22 shows the Variable frequency Drive (VFD) monitor signal from the drive motor controller prior to us making any changes. The trace represents one complete run, where the motor direction is reversed six times - the dips in signal. This graph shows only the magnitude

\footnotetext{
${ }^{13}$ As we currently perform multiple passes over one target to build up the required film thickness, we need to reciprocate the carrier across the target. This means reversing the carrier after it has passed over the target, and we do not have the required number of limit switches to control this motion. It is a simple matter to incorporate them, and this is planned for the near future.
} 
of the signal, and not the direction. The noise on this trace reflects the noise generated by the PLC.

Some time ago, it was noted that this was somewhat noisy, but decided that it was not significantly affecting the process.

Recently,

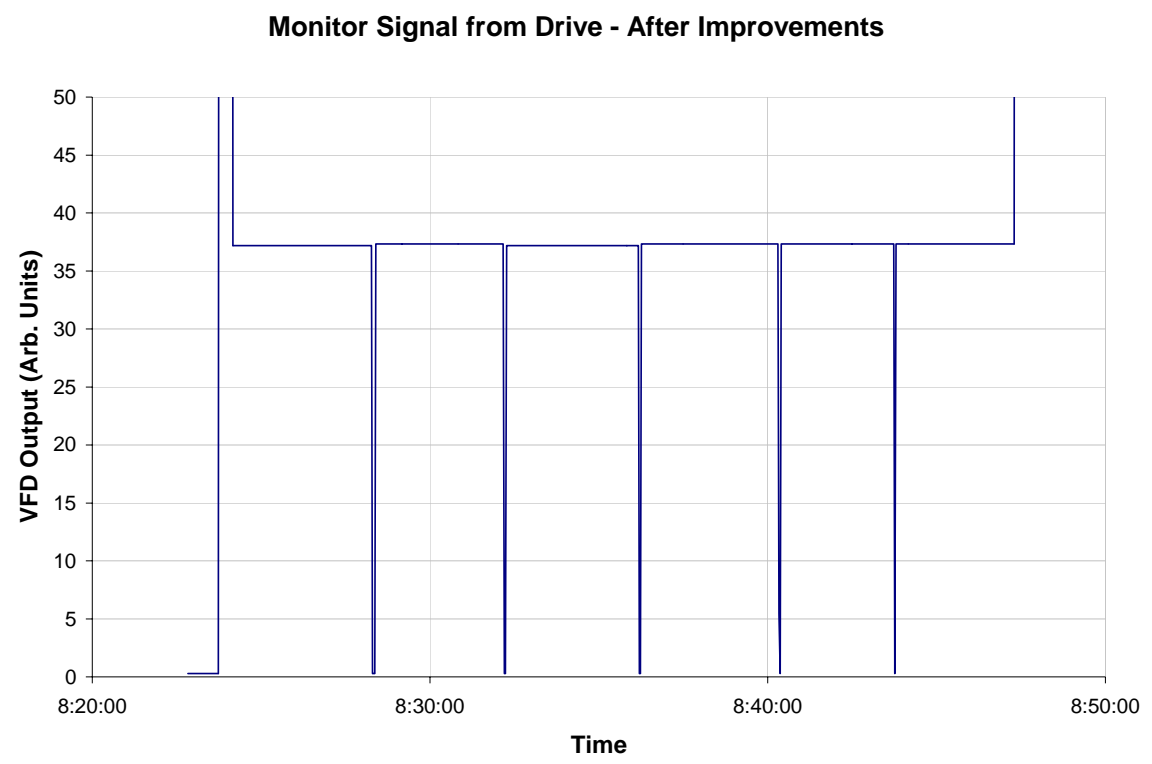
however, the noise on this signal has Figure 2.23 - Control signal for drive-speed after improvements. become much more pronounced, and was leading to linespeed changes that were visible to the operators. The decision was made to replace the signal derived from the PLC with a signal generated by a completely separate source. This signal is shown in Figure 2.23.

It is clear that the signal after improvement is significantly cleaner and less noisy than before. It is therefore anticipated that there may be an improvement in the consistency and therefore quality of the films deposited as a result. This appears to be borne out by inspection of resulting film thickness for one of our critical layers. Some data are shown in Figure 2.24, where we show the measured thickness for one of the films, before and after the change just described. There is a clear reduction in variation - from around 6\% prior to the change, to around 1\%. This is a good example of the improvements in quality which we are striving for, and this one could only have been accomplished by monitoring the drive control signal using our integrated process monitoring system. 


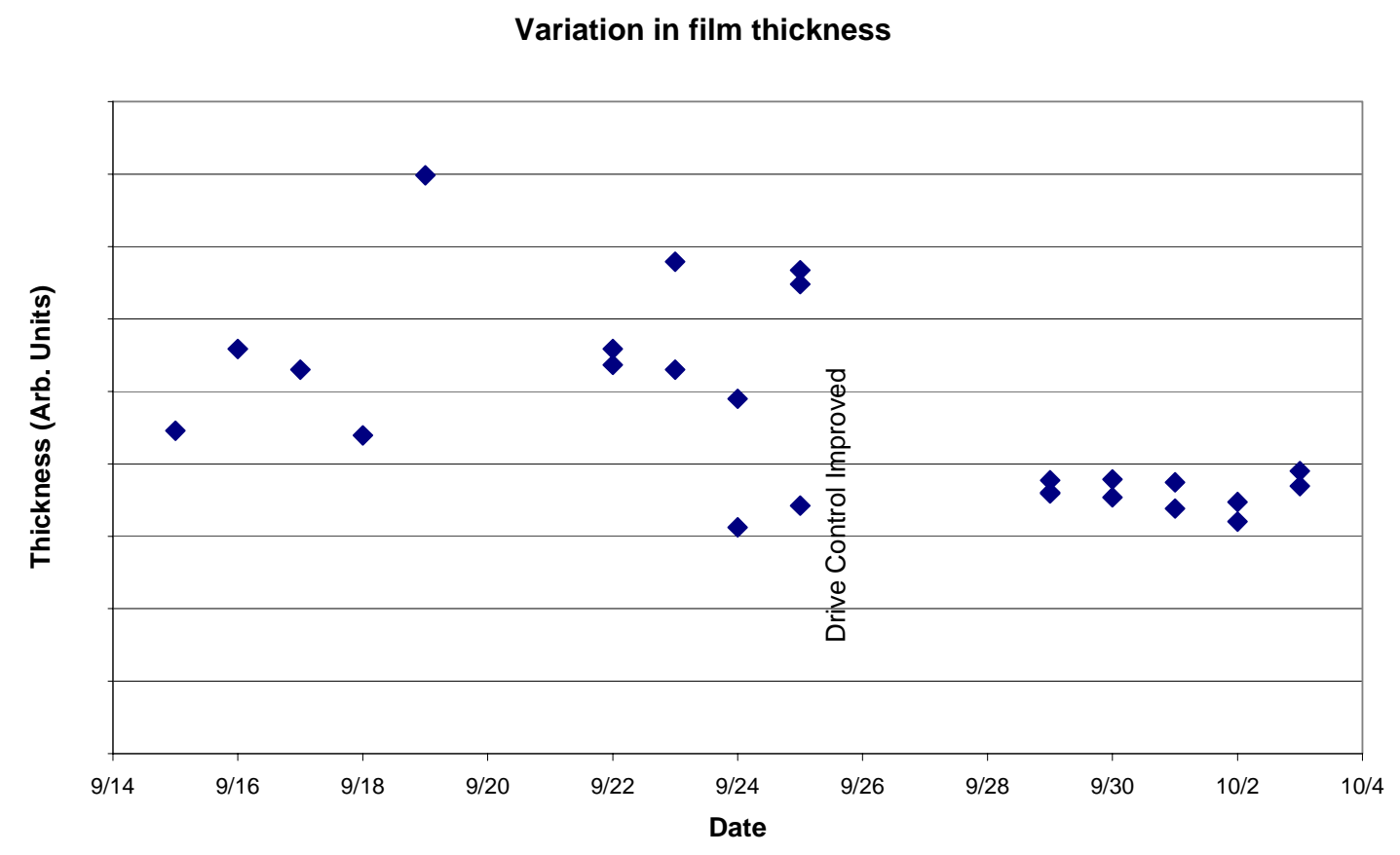

Figure 2.24 - Measurement of the thickness of one of the key layers in the EC device, both before and after the changes were made to the drive control signal.

\subsubsection{Summary of Task 4}

The objective of this task was to integrate the range of on-line inspection and monitoring techniques that were developed in Tasks 1-3. Although these have not been implemented into a fully searchable database, many outputs have been integrated into a suite of linked spreadsheets, serving a similar purpose. For example, the optical monitoring system has been integrated sufficiently to allow outputs from the optical monitor to generate control parameters for the deposition process. Currently, the loop is being closed manually, and will switch to automatic control in the future.

Process monitoring in conjunction with SPC techniques, has been seen to yield improvements in several areas, from clean room temperature control, to drive-speed monitoring. Further improvements in process control are expected as we continue to add further monitoring.

Although complete integration has not yet been achieved, enough understanding has been obtained for implementation in the next stage manufacturing plant. 


\section{Conclusions}

The work done in this project has been aimed at improvements to the process that will result in yield increases. The work has been divided into four main areas, dealing with substrate preparation, particle control, optical monitoring, and system integration.

In the area of substrate preparation, the problem has been segmented into manageable pieces. This has resulted in many improvements, not least of which is the installation of a high quality washing system, incorporating best operating practices.

Different washing techniques have also been investigated, and an optimum arrangement for reducing defects that show up in finished devices has been achieved. This required development of an inspection technique, used to find defects on the substrate before EC device production, and these defects could therefore be correlated with defects measured in finished devices.

Another significant part the substrate preparation work was the development of specifications and requirements with our glass supplier. This has resulted in numerous improvements to the quality of the as-received glass.

One really significant advancement in the area of substrate preparation has been the introduction of a quality program. The aim of this program is to ensure the highest possible level of consistency throughout all of the substrate preparation steps. This encompasses everything discussed in this report relevant to substrate preparation.

A related issue is the reduction of particle contamination sources throughout the process. Obviously, some of the substrate preparation activities impact the generation of particles, particularly in the areas of glass cutting, seaming, line isolation and bus bar application. Substantial advances in reducing the number of these particles, and their propensity to find their way into the process has been made. In addition, the issue of particle generation during sputtering has been addressed, and significant progress in understanding the origin of the contamination has been made. The reductions in contamination can be correlated with lower defect densities, particularly in white dots and shorts.

Optical monitoring is now a crucial and well-understood part of our process. Hardware has been developed, which is robust, can be integrated neatly into our vacuum system, and can make the real-time measurements required. Furthermore, the dependence of final device properties on key process characteristics has been investigated resulting in the optical monitor being used to maintain the process at the optimum point. Software systems have been designed to generate information that helps the operator make critical processing decisions. Currently the output of the optical monitor is being integrated into a suite of software programs, which will ultimately result in closed loop control of the whole fabrication process. A bonus development has been the discovery of a novel method for controlling the uniformity of one of the key layers, which depends critically on real-time feedback from the optical monitoring system. 
The work on plasma monitoring has not advanced to the same extent, but valuable progress has been made towards implementation of this technique. It is important to link plasma monitoring with the real-time uniformity control system, as this could provide advance warning of any non-uniformity in the deposition process, and so allow correction of the potential problem prior to any film deposition.

The final part of the project was concerned with integration of a variety of monitoring systems with a central database. Several hardware systems have been installed to diagnose some existing problems. This has lead to several small but significant quality improvements, e.g. identification and rectification of the drive controller problem. Further small but important incremental improvements are expected as more monitoring equipment is integrated into the system.

Implementation of the quality process throughout the organization has led to improvements in all of these areas, which have, in turn, resulted in improvements in the yield of EC devices from the Pilot Line. The program aimed at increasing the overall repeatability and reliability of the process - “The Quality Program” - is expected to give further benefits in the future. However, significant improvements during the period of this work have been seen, and this is illustrated in Figure 3. 1.

The yield is calculated using an internal specification, which has been developed as a precursor to the final specification which will be used to determine whether a product can be shipped to a customer. The current specification is fairly close to the final version, and so provides a good measurement of the actual progress. Devices made in 'experimental' runs have been excluded, as these are carried out for process development. 
Pilot Line Performance

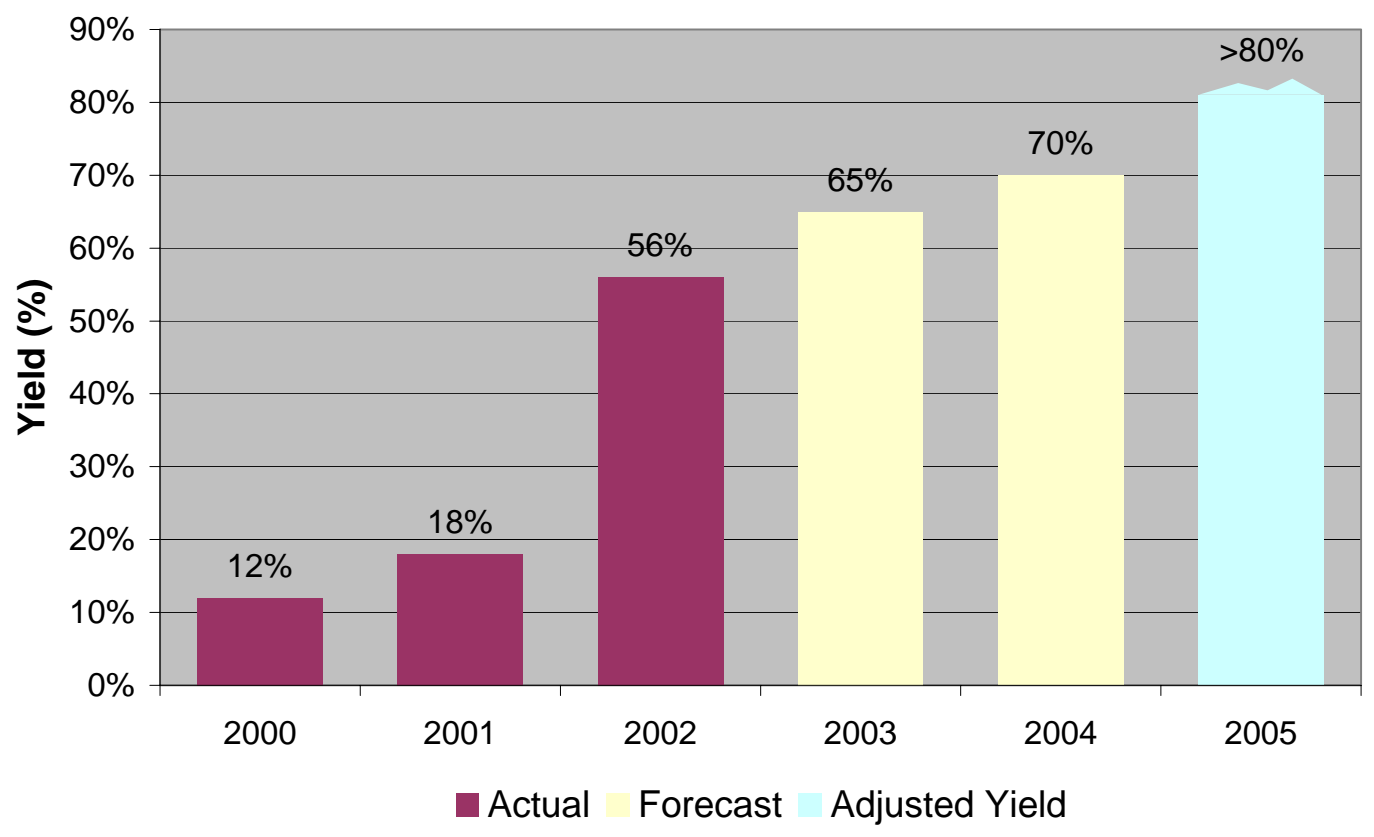

Figure 3. 1 - Overall yield for the Pilot Line both actual and predicted. The yield figure for $\mathbf{2 0 0 5}$ has been adjusted to accommodate the known equipment and logistical issues of the present Pilot Line.

There has been a clear increase in the yield occurring over the period of this project (Sept 1999 to September 2003), which is mostly attributable to the work reported here.

Although specific improvements have been made during this project, a major accomplishment has been the establishment of procedures for continual improvement. Te quality program is firmly established and will drive further yield and productivity improvements as SAGE moves to commercial manufacturing. 


\section{Bibliography}

US Dept. of Energy Core Data Book, 2001, Tables 1.1.1, 1.1.2, 1.1.3, 1.2.3, 1.2.10, 1.3.3, 1.3.9.

M.D. Rubin and R.T. Sullivan, Lawrence Berkeley National Laboratory, personal communication - energy calculations.

R. Sullivan, E.S. Lee, K. Papamichael, M. Rubin and S. Selkowitz, 'Effect of Switching Control Strategies on the Energy Performance of Electrochromic Windows,' Proc. of SPIE International Symposium on Optical Materials Technology for Energy Efficiency and Solar Energy Conversion XIII, Freiburg, Germany, April 1994.

'Progress toward durable, cost effective electrochromic window glazings', N. Sbar, M. Badding, R. Budziak, K. Cortez, L. Laby, L. Michalski, T. Ngo, S. Schulz, and K. Urbanik, Solar Energy Materials and Solar Cells, 56 (1999) 321-341

M. Feldman, R. Weissman, 'Initial stages of float glass corrosion', Journal of NonCrystalline Solids, 218 (1997) 205-209

'Understanding Statistical Process Control', D.J. Wheeler and D.S. Chambers, SPC Press (1992)

'Design and Analysis of Experiments', D.C. Montgomery, Wiley $5^{\text {th }}$ Edition (1997)

Finlay et al, US Patent 6352755, March $5^{\text {th }}$, 2002, 'Alkali metal diffusion barrier layer' 


\section{List of Acronyms and Abbreviations}

\begin{tabular}{|c|c|}
\hline APC & Adipic Acid \\
\hline $\mathrm{Ar}$ & Argon \\
\hline BSE & Backscattered secondary electron \\
\hline BTU & British Thermal Units \\
\hline $\mathrm{CE}$ & Counter-electrode \\
\hline $\mathrm{CO}_{2}$ & Carbon dioxide \\
\hline CVD & Chemical Vapor Deposition \\
\hline $\mathrm{DC}$ & Direct current \\
\hline DI & Deionized \\
\hline $\mathrm{DOE}$ & Design of Experiments \\
\hline DOE & Department of Energy \\
\hline $\mathrm{EC}$ & Electrochromic \\
\hline EDS & Energy Dispersive X-ray Analysis \\
\hline $\mathrm{F}$ & Fluorine \\
\hline FTO & Fluorine-doped Tin Oxide \\
\hline GASP & Glancing Angle Surface Polarimetry \\
\hline $\mathrm{H}^{+}$ & Hydrogen ion \\
\hline IC & Ion Conductor \\
\hline ICP - MS & Inductively Coupled Plasma Mass Spectrometry \\
\hline LAN & Local Area Network \\
\hline $\mathrm{kV}$ & Kilovolts \\
\hline $\mathrm{kW}$ & Kilowatts \\
\hline $\mathrm{N}_{2}$ & Nitrogen \\
\hline $\mathrm{O}_{2}$ & Oxygen \\
\hline $\mathrm{OH}^{-}$ & Hydroxyl Ion \\
\hline OD & Optical Density \\
\hline PC & Personal Computer \\
\hline PEM & Plasma Emission Monitoring \\
\hline PLC & Programmable Logic Controller \\
\hline $\mathrm{PM}$ & Preventative Maintenance \\
\hline ppm & Parts per million \\
\hline PVD & Physical Vapor Deposition \\
\hline Quad & Quadtrillion BTUs \\
\hline $\mathrm{RE}$ & Reference-electrode \\
\hline $\mathrm{RO}$ & Reverse Osmosis \\
\hline sccm & Standard cubic centimeters per minute \\
\hline
\end{tabular}


SE Secondary electron

SEM Scanning Electron Microscopy

Si Silicon

SIMS Secondary Ion Mass Spectroscopy

SPC Statistical Process Control

SS Stainless Steel

TC Transparent Conductor

VFD Variable Frequency Drive

WE Working-electrode 